2018

\title{
Phenomenology and Geographic Information Science: experiencing landscape through geospatial technologies and Higuchi-style indices
}

L. Jesse Rouse

Follow this and additional works at: https://researchrepository.wvu.edu/etd

\section{Recommended Citation}

Rouse, L. Jesse, "Phenomenology and Geographic Information Science: experiencing landscape through geospatial technologies and Higuchi-style indices" (2018). Graduate Theses, Dissertations, and Problem Reports. 7243.

https://researchrepository.wvu.edu/etd/7243 


\title{
Phenomenology and Geographic Information Science: experiencing landscape through geospatial technologies and Higuchi-style indices
}

\author{
L. Jesse Rouse \\ Dissertation submitted to the \\ Eberly College of Arts and Science \\ at West Virginia University \\ in partial fulfillment of the requirements for the degree of \\ Doctor of Philosophy in Geography
}

Trevor Harris $\mathrm{PhD}$, Chair

Kenneth Martis PhD

Maria Perez $\mathrm{PhD}$

Timothy A. Warner PhD

Kenneth Fones-Wolf $\mathrm{PhD}$

Department of Geology and Geography

Morgantown, West Virginia

2018

Keywords: phenomenology, landscape archaeology, cultural geography, GIS, geospatial

Copyright 2018, L. Jesse Rouse 


\title{
ABSTRACT \\ Phenomenology and Geographic Information Science: experiencing landscape through geospatial technologies and Higuchi-style indices
}

\author{
L. Jesse Rouse
}

Research conducted on cultural landscapes is often divided into two approaches comprising a qualitative description or quantitative measure. The separation is often driven by the difference in the approach and methods utilized. Phenomenology has often been used as the framework with which to consider the experience and quality of a landscape. The descriptive and personal nature of phenomenology seeks to separate the observer from their personal biases to yield a qualitative narrative. Conversely, Geographic Information Science (GIScience) is seen as a set of methods and tools to capture, map, and analyze the quantifiable features of a landscape. However, it is proposed here that a shared geographic focus on cultural landscapes that bridges these two approaches would increase the value of such studies. Providing such a bridge offers a more structured experiential and qualitative assessment of cultural landscapes which is augmented by a measured assessment implemented using GIS and begins to address issues about calculating only the visible aspects of landscapes and the difficulty of replicating phenomenological studies.

Landscape Archaeology and Cultural Geography have each taken advantage of phenomenology and GIScience as approaches to understanding cultural landscapes. In either approach, there has been an emphasis on what can be physically seen resulting in a focus on issues of landscape visibility. Visual perception and representation act as the keystones which holds up the bridge between these approaches. While previous models such as isovists, viewscapes, and viewsheds provide examples of linking visual perception and representation, they generally lack a deeper connection to cultural issues.

Other approaches exist that attempt to incorporate cultural aspects into structured models. Additional cultural aspects could be considered through resourcescapes, that focus on material culture and raw materials, or taskscapes, that capture cultural activities. However, it is the use of more involved models of landscape visibility such as those proposed by landscape architect Tadahiko Higuchi that look at the visual and spatial structure which provide a strong link between the experience of landscape and a structured approach linking visual perception and representation of landscape.

This research utilizes an example from Marietta, Ohio which centers not on the modern landscape, but the extant prehistoric mound features and the prehistoric cultural landscape they represent. The consideration of the Marietta mounds provides an example of how an experience of the extant prehistoric features and a model of the prehistoric landscape can work together to highlight the role of the perception of the observer and the model driven from the observer's perspective of the landscape. From the potential the mounds afford through the experience of walking among them to the affinity that the observer gains through the spatial models created using Higuchi's indices, bridging these two camps of cultural landscape research builds on the strengths of both approaches. 


\section{Acknowledgements}

Almost two decades have passed since I began my acknowledgements in my MA thesis, and as suggested at that point, there are more names that can be added to the list and others that must be acknowledging again. I have spent the time since my thesis in various positions and at two schools, one as a student and employee, the other as an instructor. Everyone I have known from both schools deserve a thanks in keeping me moving forward.

The most direct thank you must go to my doctoral committee who supported my completion even though there were some hoops that were required. A most effusive 'thanks' must go, again, to Dr. Trevor Harris for working with me to get to the final document even though full-time positions often distracted me. For the other committee members, Dr. Kenneth Fones-Wolf, Dr. Kenneth Martis, Dr. Maria Perez, and Dr. Timothy Warner, I greatly appreciate the support you have provided since I met each of you. I look forward to continuing our discussions at our regular conferences.

While I graduated with a great committee, I have others who were at one time members of the committee who were not in the final group due to changes in dissertation topic, changes in their position, or even retirement since I began the doctoral program. This group includes Vic Baker, Dr. Steven Kite, Dr. Gary Lock, and, of course, Dr. Briane Turley. Thank you all for your willingness to work with me.

My past and current colleagues in the Department of Geology and Geography at the University of North Carolina at Pembroke have both distracted and assisted me over the years. Thank you all for your continuing support and letting me help to create the next generation of Geographers (fine, and geoscientists too). To my students from UNCP, and WVU, thank you for letting me share my love of Geography with you, even if you weren't always quite as enthused as I was. Special note of thanks goes to all the advisees, majors, minors, and certificate holders who reminded me that I didn't have to convert everyone in the Gen Ed sections to still have an impact. 
My family has kept 'gentle' pressure on me to finish, and while they are not all still with us, I hope that they all can know that they helped me complete this degree. The love my parents, Frank and Ruby, have shown me through the years cannot be described or compared. And my brother, Bobby, isn't too bad either.

One of the things that I can blame for the delay in finishing (and there are many) is the company that was formed in 2005. VerySpatial brought some of us in the department closer together and pulled a couple into the Geography graduate program. The travel and 'work' that I have conducted with Dr. Susan Bergeron, Frank Lafone, Barb Maclennan, Jeff Dunn, and others has only deepened my excitement about Geography and geospatial technologies as we have discussed trends within, and from outside, of the discipline through the blog, videos, and podcasts. I have had a chance to talk to some amazing folks from around the world, across the discipline, and from various industries in order to build a more dynamic image of how we as geographers impact the world every day. To our readers and listeners and interviewees, thank you!

Dr. Bergeron gets the final acknowledgment this time. I simply wish to say thank you, Sue, for putting up with me, supporting me, and loving me.

Again, there are many more that deserve thanks, but they will have to wait for the next round.

\section{Jesse Rouse}




\section{Table of Contents}

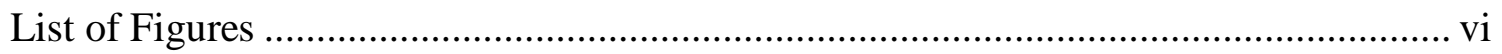

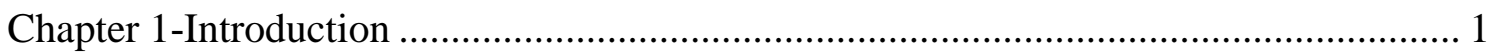

Quantitative and/or qualitative analysis ................................................................. 2

Differing Perspectives ............................................................................................ 4

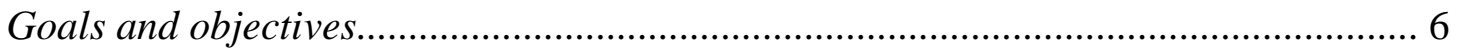

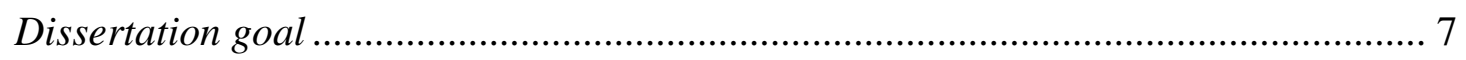

Chapter 2-Landscape, Phenomenology, and GIScience................................................ 10

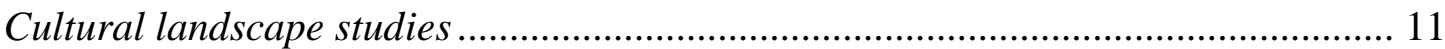

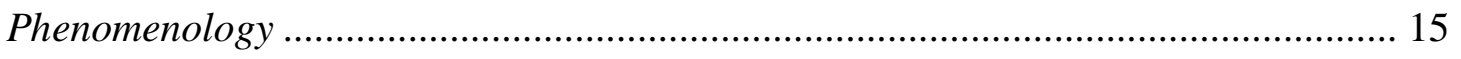

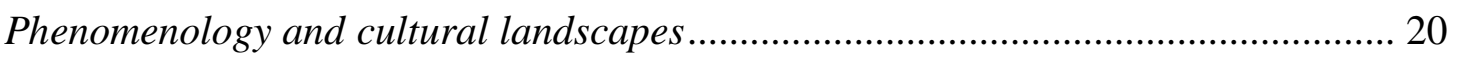

Geographic Information Science .......................................................................... 23

The role of GIS in landscape archaeology modeling ............................................... 28

Chapter 3-Perception and Visual Landscapes ............................................................... 31

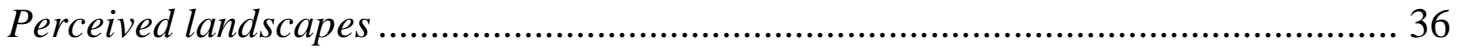

GIS and the modeling of landscapes ....................................................................... 37

Chapter 4-Combinatorial methods for exploring 'scapes ............................................... 42

Implementing 'scapes ............................................................................................. 48

Chapter 5-The Higuchi viewshed …………………….............................................. 51

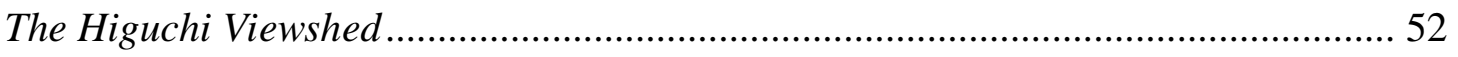

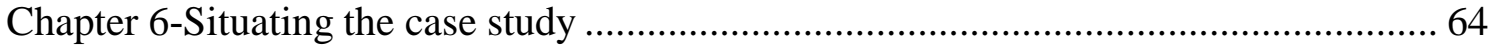

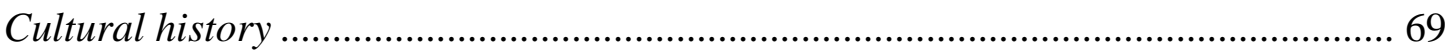

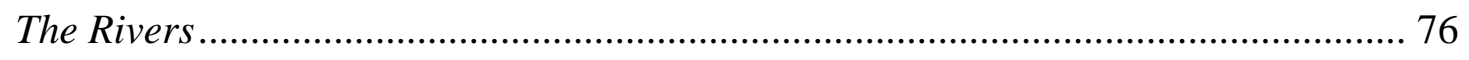

Chapter 7-Experiencing the landscape of Marietta .......................................................... 79

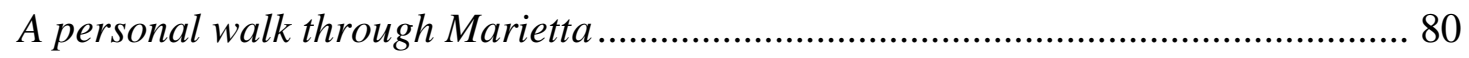

Chapter 8-GIS, Higuchi indices, and the modeling of landscape................................... 89

A Visualscape of prehistoric Marietta ....................................................................... 89

Chapter 9-Conclusion and future directions ................................................................ 103

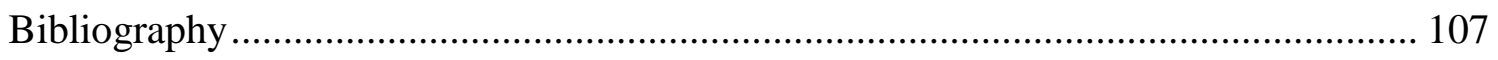




\section{List of Figures}

Figure 4.1. Small view angle of a large object from a distance...................................... 45

Figure 4.2. Large view angle of an object from near the feature...................................... 46

Figure 4.3. Material sources within the Hopewellian Interaction Sphere ......................... 47

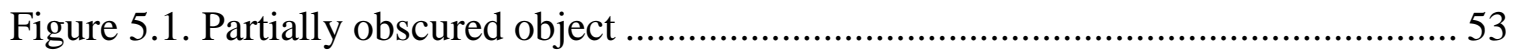

Figure 5.2. Representation of Long, Middle, and Short Distance ……............................ 55

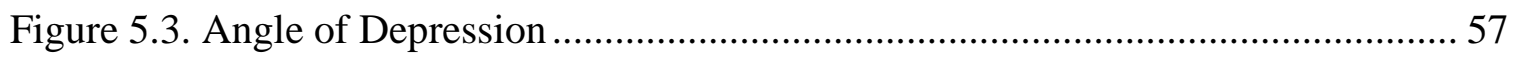

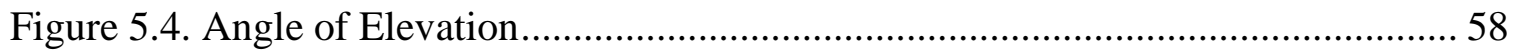

Figure 5.5 Visual range based on Higuchi ..................................................................... 59

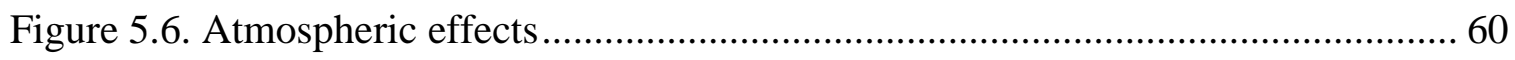

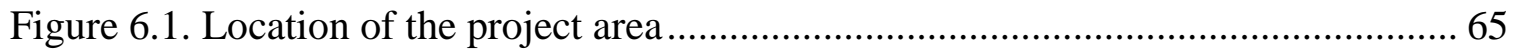

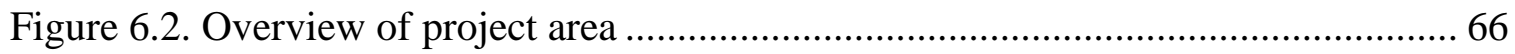

Figure 6.3. Extant mounds and recorded earthworks ..................................................... 67

Figure 6.4. Marietta Mounds (Bryant and Gay 1888) ....................................................... 68

Figure 6.5. "Plan of the Ancient Works at Marietta, Ohio," by Charles Whittlesey ........ 69

Figure 6.6. Cemetery Mound in Marietta ……………………….............................. 72

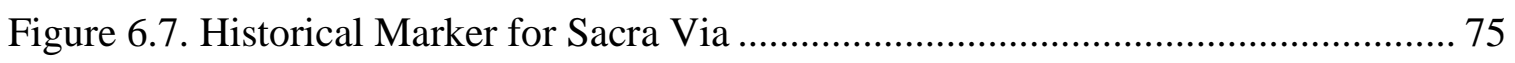

Figure 6.8. Ohio River front looking up toward downtown Marietta................................ 76

Figure 6.9. Looking across the Muskingum River toward Marietta................................. 76

Figure 6.10. Williamstown, WV looking across the Ohio River..................................... 77

Figure 7.1. Cross-section of Sacra Via from Whittlesey's 1837 survey........................... 80

Figure 7.2. Whittlesey's 1837 historic survey map of Marietta ......................................... 81

Figure 7.3. Minor remains of one of the interior embankments ..................................... 82

Figure 7.4. Truncation of one of the ramps leading up to Quadranou.............................. 83

Figure 7.5. Quadranou as seen from the southwestern corner of park .............................. 83

Figure 7.6. Capitolium and the historic library that sits on top ........................................ 84

Figure 7.7. Neighborhood of present day Capitolium .................................................... 85

Figure 7.8. Mound Cemetery and Cemetery Mound ………............................................ 85

Figure 7.9. Steps placed on the side of Cemetery Mound in the 1830s............................. 86

Figure 7.10. Modern view from atop Cemetery Mound looking west ............................. 86 
Figure 8.1. Areas visible from Cemetery Mound ................................................. 90

Figure 8.2. Distance from observer at Cemetery Mound (short to long distance)........... 91

Figure 8.3. View from bridge viewing western side of Muskingum River .................... 92

Figure 8.4. Angles of Depression and Elevation from observer at Cemetery Mound...... 93

Figure 8.5. Angle of Incidence between the terrain slope and the observation angle ...... 96

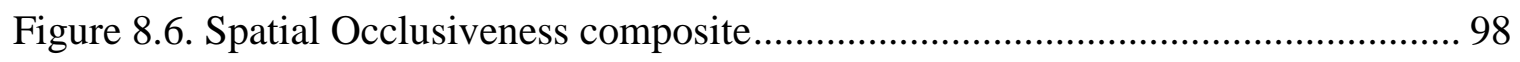

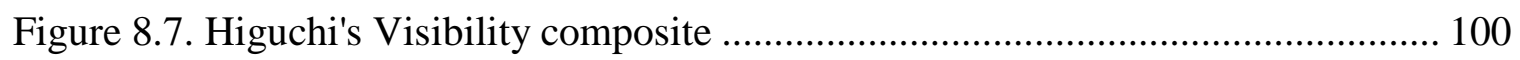




\section{Chapter 1 - Introduction}

From Carl Sauer's (1925) work on the interaction between landscape and culture, to the humanistic Geography of Yi-Fu Tuan (1977) and beyond, there has long been significant interest in cultural landscape studies that draw on phenomenology and spatial perception to understand the world we inhabit. This link between experience and spatiality is often termed the experience of landscape, whether it is an experience of an extant landscape (Higuchi 1988), a historic landscape (Sauer 1963), or even a landscape painting (Appleton 1975). The experience of landscape links the observer and the observed in order to provide an often-unique perspective of a landscape. In some cases, the perspective is used to gain deeper insight and interpretation of the landscape, while in other cases the perspective is a descriptive narrative of the time and the space observed. In either case, the core of the encounter is the experience that the observer has of the landscape.

The study of landscapes has also been connected to Geographic Information Systems (GIS), and the use of geospatial technologies has driven the development of significant methods and techniques that yield valuable insight into landscape patterns (Lock 2000; Rouse 2000). GIS is excellent in providing the researcher with the capability to capture and store observed objects, to conduct spatial analysis on those objects, and to share the results. A GIS, then, provides a framework, or structure, from which to build a model of the landscape. However, GIS does not lend itself easily to the capture or sharing of the personal nuances gained through an experience of landscape. What has been of interest for many, given that landscape is a bridge that allows for a structured experience of landscape, is to identify a way to model the landscape using GIS that either builds from an experience or that can inform an experience. 


\section{Quantitative and/or qualitative analysis}

Spatial data in GIS is invariably viewed as objective and based on an empirical approach where the intent is to acquire accurate and measured data through rigorous and replicable means. The cataloging of physical and cultural geography is important in GIS in order to consider our interconnected world, though the abstraction and objectification of real-world phenomena into spatial primitives for use in GIS can de-contextualize these phenomena in many ways. This is not to say that the conversion from phenomena to data objects is not without its value, but the connectivity between observer and the observed landscape is influenced considerably by abstracting the world through spatial primitives that can be readily used in a GIS.

From the experiential perspective, the observer/subject connection is an integral part of the experience of landscape. However, perspective is lost in abstracting the real world into spatial primitives, because it disconnects the phenomena from the context within which the phenomena was experienced. This disconnect is critical in the separation of the experience of landscape and the data traditionally used in GIS-based landscape analysis.

The search for Middle Ground in landscape archaeology (McEwan and Millican 2012) makes clear the issues driving the discussion surrounding the tensions between the quantitative data utilized in GIS and the qualitative information that describes the experience of landscape. Just as GIScience has found limitations in current methodologies and technologies, so landscape archaeology has seen the impact of these limitations play out in the use of GIS (Lock 2000). With the push toward post-processual approaches that often rely more on description than measurement, there is a tension between the conceived empirical nature of GIS and the experiential nature of phenomenology. This tension is exacerbated by those who have pursued an experiential approach in a purist manner, and where they suggest that only a personal, situated 
experience can yield a deeper knowledge. Discussions from Tilley (1994) and Thomas (2004), two of the dominant voices of an experiential landscape approach specifically, and landscape archaeology generally, seek such a focused approach. While they are not without their critics (Flemming 1999), it is clear that many see the potential of a situated experiential landscape, if not wholly according to their suggested methods. Conversely, there is a growing use of technologies from GIS to 3D scanning in landscape archaeology that focuses predominantly on the use of spatial data to ascribe inferences to sites and finds. Again, there are critics (Thomas 2004), but many would argue for the significant potential that these technologies offer to archaeology and landscape studies in general.

Perhaps it is Rennell's (2012) description of a reflexive approach to linking the experiential with the modeled representation, in which the approach does not rely on a rigid methodology but allows a response to the issues that arise, that offers the clearest description of a true mixed methods approach to landscape archaeology. While the research approach offered by the Scientific Method works well in many areas, the post-processual and post-modern trend in social science research, and the desire and need to incorporate humanistic ideas, has shown that the positivist trend toward the perceived rigor of hypothesis testing is not always the most appropriate approach. Similarly, with new techniques providing insight into the past, an approach tied purely to experience has its own set of limitations. A reflexive methodology that is informed by, and incorporates, a range of methods, techniques, tools, and technologies so as to be adaptable to the needs that the research poses is perhaps more of a reality than most researchers express in their reports. The Qualitative GIS discussions (Cope and Elwood 2009) of a mixed methods approach describe such a reflexive methodology that deals with research objectives in a situational manner, looking to the appropriate combination of methods and technologies to 
approach the research question at hand as opposed to focusing on defining a strict methodology to be employed.

\section{Differing Perspectives}

Spatial perspectives tied to experience and GIS, then, could be generally described as falling into two representative categories to define a duality comprising bodily viewpoints, what a person can see, and extensive points of viewable areas, such as a viewshed map. An embodied viewpoint captures the world directly through personal experience and the human senses. The aesthetics of landscape, for example, are perceived through an individual's perspective and are reflected and described as such. This egocentric viewpoint is cognitive, individual, and arises from experience. A counterpoint to the egocentric perspective, a geocentric viewpoint, takes into account information that is gleaned through indirect means including spatial data analysis. These geocentric perspectives are not experienced directly, but extend human perception through a wealth of media, techniques, and technologies and provide a context for an individual to evaluate a landscape without necessarily even visiting it. This geocentric view of landscape is often seen as an 'objective' perspective that exploits the data analysis and presentation capabilities available through geospatial technologies. The geocentric view contextualizes the phenomena within a larger abstraction and scientific framework and seeks relationships that are not knowable, or are not obvious, solely from an experiential or egocentric perspective.

GIS provides a means to capture knowledge of the world from a predominantly geocentric perspective. The geocentric view of the world presented through GIS is made up of spatial objects stored in a database and reproduced predominantly through the lens of a cartographic product. Conversely, our embodied, or egocentric, experience of the world is that 
which we perceive directly or that which we describe, often textually or graphically. It is in the meeting of the geocentric view offered by geospatial technologies and the egocentric perspective of the embodied experience that partially allows us to engage in an experience of landscape through GIS and provide a way to move beyond our physically limited perception to better understand broader patterns within landscapes.

In the blending of humanistic and technological approaches to landscape, attention has often focused on the manner in which geospatial technologies have been used in landscape studies. There have been questions raised about these technologies and the data used within them that suggest geospatial technologies sit within an objective vacuum divorced from the people they capture, the purpose for which they were created, and the culture in which they were created (Schuurman and Leszczynski 2006). In addition, it is often assumed that with technology the ontological focus is centered on the computer screen. GIS does offer options that bring an embodied perspective to analysis such as through the use of viewshed analysis that can provide a linkage between the egocentric and geocentric perspectives. A traditional viewshed analysis, for instance, is based on a terrain model and the location of a virtual observer and provides a product that represents areas of the terrain that are potentially visible to an observer along with those areas that are not visible (Wheatley 1995). What is missing from GIS terrain analysis, and what is obvious to anyone standing in a landscape, is the powerful personal experience that heavily influences our preferences, appreciation, and assessment of the landscape itself.

Whether discussing traditional viewsheds, intervisibility analysis, or isovists (Batty 2001), not all viewsheds are created or interpreted equally. Tadahiko Higuchi (1988), in his landmark book The Visual and Spatial Structure of Landscape, examines the role of visibility in measuring the aesthetics of Japanese landscapes. His thinking extended well beyond the 
traditional concept of a viewshed as he sought to merge measures of the physical environment, human physiology, and the aesthetics of landscape. Importantly, Higuchi considered not only the physiological perception of viewsheds, but also landscape types and the location and placement of cultural features within landscapes. Higuchi's viewsheds provide a basis for measuring aspects of the landscape that are important in fusing egocentric and geocentric perspectives to landscape. Utilizing Higuchi's viewsheds in connection with GIS it is perhaps possible to gain both a sense of landscape intervisibility and attain elements of a user experienced landscape.

In considering how experience and representation can come together, what remains ambiguous is how best to bridge these two approaches in a way that will yield a robust, if loosely coupled, interpretation of a landscape. By pursuing a personal experience of landscape with representations of landscape offered through spatial data and models, a structured experience could provide a potential framework with which to gain an egocentric response to a landscape while simultaneously affording a broader geocentric understanding to the observer. In doing so, the observer could link the modeled analysis of the landscape with their examination of the landscape to support and bolster the interpretations that can be made.

\section{Goals and objectives}

The goal of this dissertation is to consider and develop a connection that links the experiential, phenomenological approach to cultural landscape studies with the geospatial technologies and data that underpin Geographic Information Science. In so doing, this study seeks to draw together two seemingly disparate areas of research, phenomenology and Geographic Information Science, in order to provide additional insight into landscape analysis and interpretation. The study is guided by models derived from the physiologically oriented Higuchi viewshed approach that 
reflects the human experience, and to extend it to include geocentric perspectives through the use of resourcescapes, taskscapes, and visualscapes (Ingold 1993; Epstein and Axtell 1996; Llobera 2003).

To pursue such an approach, it is necessary to go beyond the traditional quantitative measures of archaeological evidence used in GIS studies and to interweave the qualitative information and human experience that are often excluded from GIS analysis. Through the use of Higuchi-based indices and GIScience, along with the experience of landscape, this study has the potential to bridge the divide between processual spatial science and post-processual interpretive approaches to cultural landscape investigation.

\section{Dissertation goal}

The goal of this research is to explore and develop a structured experiential and phenomenological approach to prehistoric landscapes through the linkage of Higuchi viewsheds and geospatial technologies. The specific objectives and tasks are:

1) Review the literature on:
a) existing theory and methodologies used to explore landscape,
b) geospatial technologies in cultural landscape studies,
c) phenomenology and landscape, and
d) Higuchi viewsheds. 
2) Critique conventional viewshed analysis and related spatial techniques including:
a) viewshed analysis,
b) intervisibility,
c) visual indexes,
d) and isovists.

3) Develop a conceptual model to link phenomenology, geospatial technologies, and cultural landscapes:

a) Adapt, amend, and add to Higuchi's viewshed indices to create a model to support a structured experiential approach to prehistoric cultural landscapes,

b) Insert specific indices based on taskscapes, resourcescapes, and symbology,

c) Consider egocentric and geocentric perspectives.

4) Develop GIS-supported Higuchi-based indices to study prehistoric landscapes by:

a) Embedding existing Higuchi indices within GIS

b) Establishing cultural indices that blend the spatial assessment of landscapes with interpretations of prehistoric life experience, and

c) Coupling the GIS model results with personal and expert experiences to interpret landscapes in a way that links egocentric and geocentric landscape perspectives.

5) Implement these indices through a case study based on a prehistoric cultural landscape in Marietta, Ohio by:

a) Utilizing landscape and physiologically derived information, and

b) Assessing how quantitative indices differ from expert/personal experience. 
6) Evaluate the use of structured indices to support an experiential landscape archaeology to:

a) Based on insights gained from the case study understand the role and importance of both visual and experiential forms of interpretation,

b) Determine how well the indices support a phenomenological approach to understanding past cultural landscapes,

c) Determine future research avenues for structured indices in landscape analysis.

Through the following chapters these objectives and associated tasks provide a basis for grappling with the issues and potential for creating a structured experience of landscape. Elements of the experience of landscape, phenomenology, and Geographic Information Science are outlined to inform the theoretical foundation of the research (Chapter 2). An overview of work conducted on perception and visual landscapes, as well as related analyses, is provided as a basis for extending these methods in Chapter 3. The basis of various methodologies that consider the geocentric perspective of landscape is provided in Chapter 4 and the implementation of the research built on Higuchi indices is detailed in Chapter 5. The case study setting and cultural history of Marietta, Ohio is outlined in Chapter 6 and a detailed personal experience of the case study area is presented in Chapter 7. The application of a model that combines the experience of landscape with spatial modeling demonstrates the theoretical and methodological implementation and operationalization of the approach (Chapter 8). Finally, the research brings together these several threads in order to suggest a connection between phenomenology and Geographic Information Science to enhance a humanistic GIS interpretation of cultural landscapes. 


\section{Chapter 2 - Landscape, Phenomenology, and GIScience}

Landscape concepts and the 'experience of landscape' have received substantial attention for over a century and the theme of landscape continues to capture the imagination of geographers and researchers studying cultural landscapes (Sauer 1925; Relph 1970; Tuan 1974; Lowenthal 1975; Tilley 1994; Tilley 2010). The duality of studying both the morphology of landscape and its associated cultural attachment(s) has attracted a variety of researchers to the field. The question of how to capture something as subjective as 'experience' encourages researchers to continually seek out new methodologies and theories to drive this seemingly intangible line of enquiry. Much of the research into the experience of landscape has been, and continues to be, based on a phenomenological approach to understanding the world.

Attempts to capture the experience of landscape in the U.S. can be traced back to Sauer's (1925) discussion of cultural geography and phenomenology as a means to explain features that exist within a landscape. Today, with the growing use of geospatial technologies to capture, represent, and analyze landscapes, the phenomenology of landscape has largely been set aside in favor of more quantitative geospatial approaches. This seeming separation between experience and technology has altered the way in which we approach and interpret cultural landscapes. However, in this dissertation it is proposed that opportunities exist to blend qualitative research with Geographic Information Systems (GIS) in order to create a mixed methods approach that can provide the best of both a quantitative approach and incorporate a qualitative experience of landscape. 


\section{Cultural landscape studies}

In the early $20^{\text {th }}$ century, the father of the 'Berkeley School' of Cultural Geography, Carl Sauer, championed the concept of landscape in his article "The Morphology of Landscape" (Sauer 1925). In his paper, Sauer built on the German concept of landschaft, and in so doing established a distinctly geographical perspective whereby landscape became a unifying theme in Cultural Geography. Interestingly, many of Sauer's studies on cultural landscape focused on archaeology, anthropology, and the human alteration of prehistoric landscapes. This interest in a cultural attachment to place is likely tied to Sauer's links to such colleagues as Alfred Kroeber and other prominent Anthropologists at the University of California, Berkeley (Johnston 1994). While Sauer's work was influenced by the German geographical concept of landschaft, he brought a distinct cultural perspective to landscape studies that fundamentally linked culture to landscape, thereby making landscapes more than containers for physical features. Sauer examined how the landscape impacted culture (Sauer 1925) and conversely how cultures impacted landscape (Sauer 1963). Following Sauer's conceptual breakthrough, cultural landscape features and objects have been considered not solely as individual or isolated physical and human entities, but as parts of a holistic and enduring cultural landscape.

Subsequent to the seminal work of Sauer, the intellectual emphasis on landscape shifted to the United Kingdom where landscape historians followed in the steps of William Hoskins (1970) and H. C. Darby (1973). Hoskins, a prolific writer on local history, is best known for his 1955 text The Making of the English Landscape (Hoskins 1970). In this account of the regional variation of landscapes in the United Kingdom through a historical geography perspective, Hoskins moved beyond reliance on written documents as the sole source of historic information and looked to the landscape itself as a source in its own right waiting to be read like a book. In 
reference to the English landscape Hoskins writes that " $[\mathrm{t}]$ here are discoveries to be made in it for which no written documents exist, or have ever existed.” (Hoskins 1970:14). Through his words and actions Hoskins moved many to approach landscape studies differently, to experience them first hand, and to search for the stories embedded in them.

Early research into landscape archaeology combined cultural landscape studies with the historic landscape movement. Today, landscape archaeology focuses primarily on the interrelation between the physical environment and the situated meaning of cultural remains (Ashmore and Knapp 1999; Tilley 2010). Landscape archaeology can, however, be traced back further than its current incarnation would suggest. Through much of its history a focus on intrasite and intersite relationships were neither called landscape archaeology, nor were they conducted necessarily by archaeologists. If in recent years the phrase 'landscape archaeology' has been taken to mean the holistic study of a material culture in the context of landscape scale studies through a spatial focus, then landscape archaeology may even predate contemporary notions of archaeology itself as a formal discipline. Thomas Jefferson, for example, in the late 18th century is documented as having studied earthen mounds in the U.S. (Trigger 1989). Jefferson's interest in the earthen monuments of the eastern U.S. was similarly pursued by several others including antiquarians Squire and Davis in the early 19th century who recorded many of the mounds in, and surrounding, the state of Ohio (Squire and Davis 1998). These surveys contain invaluable measurements of the earthworks and mounds that in many instances are the only remaining records of the prehistoric cultural landscape that has been so heavily denuded over the past two centuries by agriculture, urban expansion, and construction projects.

Modern landscape archaeology arose in the United Kingdom in the 1960s to 1970s (Johnson 2006) and was solidified with the publication of Landscape Archaeology: an 
introduction to fieldwork techniques on post-Roman landscapes by Aston and Rowley (1974). It was Aston and Rowly who offered the first step toward formalizing landscape archaeology as a field of study (Taylor et al. 1998). Though landscape archaeology still resembled earlier landscape histories as undertaken by Hoskins (1970), landscape archaeology now reflected a more distinct cultural focus. During the theoretical and methodological debates in the social sciences that took place in the 1970s and 1980s, archaeology both embraced and confronted the growing role of positivism in the study of archaeology. During this transition, archaeology borrowed many concepts, methods, and techniques from Geography. Clarke (1972) was a notable proponent of spatial analysis in archaeology with his Models in Archaeology, a text that became the archaeological companion and equivalent to Chorley and Haggett's (1967) Models in Geography. Despite the uptake of GIS in archaeology during the late 1980s and early 1990s (Allen et. al. 1990; Lock and Stančič 1995), there was nevertheless a distinct counter movement to the positivist framework of processualism, and spatial science by proxy, in the movement toward a post-processual/postmodern perspective.

Processualism sought methods to support archaeological research following the Scientific Method in a search for laws and replicability (Trigger 1989). Post-processualism began as a counterpoint to the positivist ideas of processualism and sought to engage theory and concepts emerging within postmodernism (Hodder 1991). This post-processual counter movement rendered the broader archaeological community epistemologically splintered (Hodder 1991). In retrospect, however, it could be argued that this splintering had a strengthening effect within the discipline and that once the initial backlash against the positivist movement had subsided, researchers armed with the tools of processual archaeology sought new ways to bridge 
postmodern ideologies with the quantified remains of the archaeological material record (Preucel 1991).

Several different camps emerged within landscape archaeology during the 1980s and 1990s as processual and post-processual ideologies reverberated throughout archaeology. One prominent example that bridges the two ideologies of processualism and post-processualism is that of Hodder's (1987) Contextual Archaeology, which focuses not only on material remains and the methods utilized in their recovery, but also on the individuals who played a part in their discovery, excavation, analysis, and interpretation. This appropriately named perspective places the materials and their surroundings within the context of their recovery, an important aspect in archaeology that intrinsically binds the strengths and weaknesses of the data collection process to the recovery and interpretation of the artifacts, features, and site. Another perspective that has impacted landscape archaeology focuses more on the symbology (Nash 1997) found within prehistoric landscapes as exemplified by Tacon's (1999) study of aboriginal rock art in prehistoric cultural landscapes. This semiotic approach reads the landscape as a storied page and the objects contained therein as the archaeological 'words' that give the landscape meaning. More recently, a significant trend in landscape archaeology is to incorporate geospatial technologies, especially GIS (Chapman 2006; Lock 2003; McEwan and Millican 2012; Wheatley and Gillings 2002). GIS has provided a means to capture and share information about the physical landscape and the cultural features of landscape, as well as analyze and model spatial patterns and processes operating in the landscape. As the name suggests, these geospatial technologies are tied to geographic techniques that enable the capture, storage, analysis, and sharing of information about the landscape in a system that can provide spatial context to the phenomena in the landscape. However, the important extension into spatial thinking is, perhaps, 
less well emphasized or developed. The quantitative focus of GIS, for example, has limitations when considering any experience of a cultural landscape. The capture, investigation, and analysis of cultural landscapes using geospatial technologies necessitates a digital framework that abstracts and categorizes the landscape and essentially removes the human experience and individual interaction with that landscape. Instead, as spatial data is captured, it commonly loses its human dimension as it is reduced to a record of codified and classified spatial objects and attributes.

\section{Phenomenology}

In his "Morphology of Landscape", Sauer (1925) spoke of lebenswelt', or lifeworld, that outlines the space that is the central portion of the human experience. The prescience of Sauer in proposing the linkage of the human experience to the physical landscape and the material remains of society became apparent as his ideas subsequently found expression in the work of geographers, archaeologists, anthropologists, and others (Kroeber 1934; Tuan 1977; Cosgrove 1998; van Dommelen 1999). These ideas, which were brought to common use by Sauer in the U.S., were built on the phenomenology of German philosopher Edmund Husserl. Phenomenology can be described as a philosophical approach based on reflection and the description of personal experience of an object or landscape. Modern phenomenological research is comprised of multiple schools of thought, each of which offers a different perspective, but all of which have grown out of the work undertaken by Husserl in the early twentieth century. Four of the schools of phenomenology are Transcendental Phenomenology, Hermeneutic Phenomenology, Existential Phenomenology, and Semiotic Phenomenology and each is based on the work of an influential phenomenologist and each builds on the ideas laid out by previous 
schools of phenomenology. While Husserl is commonly recognized as the father of modern phenomenology (Macann 1993, Moran 2000) there was significant research undertaken under the name of phenomenology by earlier philosophers such as Kant who used the term to draw "a distinction between what is simply apparent, or what appears to be true, and what is in fact true." (Rockmore 2003: 88). In The Phenomenology of Mind, Hegel (1931) reflected on the ideas raised by Kant, but offered an experiential approach in which phenomena is the sole and only source of truth. These earlier considerations of phenomenology do not have direct adoption in Geography or archaeology, but they do act as a background for those 20th century philosophers who drive much of phenomenological ontology in the modern social sciences and the humanities.

The works of Edmund Husserl have had a profound impact on the development of phenomenology as a philosophical tradition (Moran 2000). Husserl's transcendental phenomenology focused on the notion of phenomenology as a philosophical method as well as a theoretical conception (Macann 1993). Husserl's phenomenological method focused on bracketing consciousness in order to focus on the essence of those objects, or phenomena, under consideration. Bracketing, as Velarde-Mayol (2000: 48) describes, is the "abstention of judging of the existence" of phenomena by which he means that while observing a feature the phenomenologist focuses on what is perceived by the act of consciousness. This focus on consciousness runs counter to a 'natural attitude' in which the "certainty of the existence of the object" is accepted (Velarde-Mayol 2000: 49). By bracketing phenomena, the observer moves away from the existential acceptance of a physical object and considers how our consciousness reflects on the object and the essence of that object. This is where many people disconnect from phenomenology. It is perhaps odd, at best, to avoid discussing what is conceived as reality and to focus internally on the experience at hand. Phenomenology, as Husserl claims, does not deny the 
existence of facts in the world, as first might be suspected. To conduct reflection and description within a method devoid of preexisting thoughts and ideas, Husserl exploited the reduction of the real into its essence and Transcendental Reduction is distinguished by its direct connection between phenomena and the ego or consciousness (Macann 1993).

There are many ideas and components to transcendental phenomenology, though the three key concepts of Husserl, the hyle, noesis, and noema, are critical. The hyle is the sensory information that is perceived by the consciousness. Examples might include the sound of a river, the view of a tree, or even the feel when touching grass. Each of these represents a direct sensory input which can be captured within human consciousness. The process that leads to creating meaning is referred to as noesis and the meaning conferred to the phenomena is the noema. Velarde-Mayol (2000) suggests that Husserl is most focused on the noesis as it represents the way in which conscious reflection creates meaning from an experience. The consideration of hyle, noesis, and noema in combination offers a focus for the reflection, analysis, and description of phenomena. These concepts and the ontology they support are what drives Husserl's perspective on phenomenology through much of his writings.

Husserl's most noted (and perhaps notorious) student was Martin Heidegger. While Heidegger adopted his interest in phenomenology from his mentor Husserl, he quickly moved to a view of phenomenology that can best be described as hermeneutic phenomenology. In his primary text, Being and Time, Heidegger (1962) focused on the concept of being. Unlike Husserl, Heidegger saw phenomenology as the self-manifestation of phenomena; "For Heidegger, phenomenology is the attempt to make manifest the matters... as they manifest themselves" (Moran 2000: 227). That is, phenomenology does not yield to a strict method, but relies on a range of methods to understand an experience. 
Heidegger's most noted concept is that of dasein, or being there. For Heidegger dasein took the place of terms such as 'consciousness' and 'world' as used by Husserl. As Malpas (2006) discusses, while the $d a$ of dasein can be translated as here or there, it was not Heidegger's intention that this would be a literal location, but rather that the term should be tied to a concept about existence. The concept of being-in-the-world (dasein) distances the Being from the phenomena that is being observed. However, the Being and its relationships to the lived world are at the core of Heidegger's phenomenology. Heidegger's work has remained at the fore for many geographers who apply phenomenology in the social sciences as an analytical approach that allows for presupposition and assumes change. Perhaps the most interesting writer on Heidegger's connection to spatial thought is Jeff Malpas who has authored and edited several texts on phenomenology and space including Place and Experience: A Philosophical Topography (Malpas 1999), Heidegger's Topology: Being, Place, World (Malpas 2006) and Heidegger and the Thinking of Place (Malpas 2012). Malpas' work provides a description of the philosophical underpinnings of phenomenology while highlighting the inherent spatial or geographic nature of different phenomenological works.

The openness provided through Heidegger's approach had a tremendous impact on later philosophers such as Sartre, Merleau-Ponty, Derrida, and Foucault. In the 1940s the intellectual focus on phenomenology shifted from Germany to France through the works of Jean-Paul Sartre and Maurice Merleau-Ponty (Macann 1993). Sartre continued his work on the existentialist path that Heidegger had laid out while Merleau-Ponty looked longitudinally across earlier schools of phenomenology. This is not to say that either Sartre or Merleau-Ponty continued the agenda set out by their predecessors since Sartre took an almost literary approach toward phenomenology 
(Sartre 1969) while Merleau-Ponty explored the linkage between consciousness and the body and how this dialectic influenced perception (Merleau-Ponty 2002).

While Heidegger has received significant attention in Geography, Merleau-Ponty perhaps has the most to say that is directly relevant to the geographer. To a greater extent than any other phenomenologist, Merleau-Ponty criticized empirical and intellectual (rational) thought. He also disagreed with the notion that consciousness previously studied within phenomenology, and as suggested by Husserl's transcendental phenomenology, stood outside of the world. Instead, Merleau-Ponty saw the body as the connection between consciousness and the world. For him, being-in-the-world, dasein, is a bodily experience in which movement creates our sense of spatiality as well as connecting us, via internalized relationships, to objects we encounter in the world. Objects that we perceive, however, may never be fully described, thereby leaving “ambiguity an inherent feature of the lived world..." (Peet 1998: 43).

Merleau-Ponty's work has been described as Semiotic Phenomenology due to its focus on language as a construction of an embodied mind. As Tilley (2004: 26) summarizes, "[1]anguage has meaning to a speaker produced through word structure." This contextualization of meaning is connected to our actions and socialization. In the end, it may be Merleau-Ponty's Phenomenology of Perception (2002) that explicitly offers a phenomenological approach for geographers through his discussion of an embodied spatiality and his exploration of Heidegger's work on the role of temporalization in experience.

Geographers and others interested in landscape, however, continue to draw from Husserl's earliest work especially through Sauer's writings. This interest may be tied to the preference of geographers to walk amongst the world and to base their discussion and analysis on the world in which we all live. For many geographers there is a connection with the world that 
almost seems to necessitate meditation and reflection through an experience of landscape (Appleton 1975). Meditation and landscape is, for many, most prominent when confronted with the relict features of a past landscape. Reflection on a ruined wall protecting what is little more than a pile of rocks inspires us to describe not only the modern setting but also what may have been. The landscape becomes a visual text that the observer translates to the written word through the methods that have been provided by phenomenologists and that mix observation and interpretation. Geographers, historians, and archaeologists alike, have adopted this metaphor of text to link landscape and phenomenology as they consider the relict remains that await them through experiencing or excavating the past (Barnes and Duncan 1992; Nash 1997).

\section{Phenomenology and cultural landscapes}

Phenomenology came to the fore in landscape archaeology in the early 1990s through the work of Ingold (1993), Thomas (1993), and Thomas and Tilley (1993). Ingold’s (1993) “The Temporality of the Landscape" owed much to the work of geographers such as Denis Cosgrove (1989) and the work of Heidegger. Ingold's simple use of Heidegger's 'to build is to dwell' and Ingold's focus on understanding the significance of daily tasks provides a stimulating insight into the power of a phenomenological approach to spatial and temporal interpretation in archaeology. The papers by Thomas (1993) and Thomas and Tilley (1993), and Tilley's (1994) landmark text A Phenomenology of Landscape, focused on a Heideggerian perspective of phenomenology and these studies have provided unique experiential understandings of the prehistoric world. These authors owed a great deal to the geographer Yi-Fu Tuan who laid the basis for a modern phenomenological perspective of place. Bender extended these ideas in landscape archaeology when she identified the "need to mesh an understanding of embodied landscapes with a political 
landscape of unequal power relations ..[and].. the need to mesh [Raymond] Williams' 'structured feeling' with a phenomenological approach.” (Bender 1999: 38). Thomas (1993, 1996), however, considered phenomenology to be part of a larger interpretive archaeology that is driven from the hermeneutic method and embraces a multivocal perspective, whereas Tilley (1994) focused more directly on the linkage between phenomenology and landscape interpretation through an exploration of 'natural' prehistoric landscape features.

Phenomenology as an approach in landscape archaeology has had its critics. Fleming (1999), for example, questioned Tilley's A Phenomenology of Landscape for its lack of observational rigor or alternative interpretations. To counter these concerns, proponents of a phenomenological approach to archaeology have sought to temper the personal/unique experience of landscape provided by a Heideggerian perspective with the ideas of Edmund Husserl and Merleau-Ponty (Tilley 2004, Tilley and Bennet 2008, Tilley 2010). This broader perspective has shifted phenomenology away from the perceived stance of being in opposition to scientific inquiry. While the experiential nature of phenomenology does not explicitly incorporate the Scientific Method, a phenomenological approach does not have to operate alone. It is a challenge to loosely couple structured analysis with personal experience to interpret cultural landscapes, but it is arguably a challenge worthy of pursuit.

To capture and represent the dialectic between technology and experience, as well as the blending of egocentric and geocentric worlds, a joining of concepts and methodologies must take place. It is Merleau-Ponty's perspective, one that sits somewhere between the Husserlian and Heideggerian, which allows a situatedness within the landscape and a disconnectedness from the landscape that has found interest in landscape research (Tilley 2004). The ability to view the world as a series of symbols is analogous to reading the landscape (Lanigan 1988). This cliché 
has merit in that the series of features and objects in a landscape are invariably perceived as representations of the subject/object dialectic within that landscape. The features and objects perceived as symbols act as the text on the landscape's paper:

"Experiencing places in the landscape involves taking as much account of the landscape in which the place is embedded, its relationship with its physical and topographical context, as of the place itself. Throughout, we assume that what makes the place significant is its relationship with other 'natural' and 'cultural' places. We are thus concerned with the dialectics of place and surroundings. Methodologically, this requires sensing place from without and from within from a variety of vantage points and pathways. No adequate understanding of the social and cultural geography of a place can be achieved without considering its relationship with others and experiencing its situation in the landscape at a human scale requiring moving and walking through and exploring its surroundings." (Tilley 2004: 221)

It is argued in this dissertation that the semiotic similarity of the perspective of experience as symbol from an egocentric viewpoint and the representation of the world symbolically through geocentric data and maps allows for a combination of viewpoints in ways that provide a needed connection between experiencing landscape and the geospatial analysis of landscape. 


\section{Geographic Information Science}

The wealth of geospatial technologies including Geographic Information Systems, Global Positioning Systems (GPS), Remote Sensing, and other spatially driven digital tools, have been used extensively to provide an environmentally structured analysis for cultural landscape research (Sheets \& McKee 1994; Llobera 1996; Aldenderfer and Maschner 1996). GIS has been heavily used to examine intrasite and intersite archaeological relationships (Kvamme 1990; Kvamme 1996; Forte 2000); to explicitly use its spatial data handling capabilities to support cultural landscape research (Lock 2003; Wheatley and Gillings 2002); and to raise a number of issues driven by how cultural landscape information is recorded and used in analysis and interpretation (Lock and Harris 1996; Wheatley and Gillings 2000; Llobera 2003). Geospatial technologies and their spatial data and models, premised as they are on spatial primitives and topological relationships, are ideal for assessing complex landscapes based on observable and quantifiable phenomena. GIS is less well situated, however, to handle the qualitative aspects that impart cultural significance to the objects and the landscapes in which they exist (Cooper and Gregory 2010).

From the early development of GIS and its roots in the cartographic tradition, there have always been challenges as to how best to abstract the 'real' world. The digital capture of geographic information in layers was brought about as a metaphor to digitally recreate the use of Mylar overlays previously utilized to track and display representations of spatial information contained in a landscape (McHarg 1969). In one of the earliest GIS projects, the Canadian Land Information System, the layers were comprised of physical information and forest reserves. In true Turing Machine and binary logic fashion, information was encoded as present or absent (1 or 0) for each cell of a gridded area (Longley et al. 2005). This inherently quantitative data capture 
began the trend that would contribute to what we recognize today as a raster GIS. Beyond the digital nature of the data structure, the positivist and reductionist nature of GIS has undeniably reinforced the tendency to quantify and categorize the elements captured in GIS data layers.

By the 1980s GIS technology was capable of handling the growing availability of spatial data. Databases stored categorized data in a GIS joined to the related descriptive information about the spatial features. These database joins were part of the adoption of modern data models and provided an opportunity for descriptive attribute information to be stored with the spatial feature. The combination of vectors and relational databases laid the foundation for spatial datasets such as the U.S. Census Bureau's census data and TIGER files (Longley et al. 2005), the National Resource Conservation Service's soils datasets, STATSGO and SSURGO (NRCS 2011) and many others. The ability to work in either a raster or vector environment increased the options available in conducting research and analysis in landscape studies (Longley et al. 2005).

As interest in GIS and related technologies flourished, attention shifted toward method and theory. The basis for a Geographic Information Science (GIScience) was laid out through a series of articles, editorials, and conversations that played out primarily in the discipline of Geography with an occasional incursion from outside. Questions of whether GIS was a tool or a science (Goodchild 1992), the theoretical underpinnings of GIS (Wright et al. 1997), and how to capture participatory information (Harris and Weiner 1996) were first discussed over 20 years ago and continue in many cases to underpin the GIScience research agenda. In the 1990s GIS had truly gained traction through the building of strong methodologies and discussions about how GIS fit within the broader contexts of theory and societal impact. As a result, GIScience made an important research shift that was broader than the previous focus solely on the technology and application of GIS. Goodchild (1992) posited specific elements in his assessment of what 
GIScience entails. These themes included data, analysis, representation, and especially conceptual and theoretical constructs. The integration of technologies and data from GIS, GPS, and Remote Sensing (Thurston et al. 2003); the analysis of data through spatial statistics and a growing set of GIS capabilities (Maguire et al. 2005); and the display of information from traditional printed representations to ever evolving digital geovisualizations (Dodge et al. 2008) has driven GIScience beyond consideration of geospatial technologies and the data that they support, toward a rich set of ontologies and methodologies.

The spatial nature of material objects and features in a landscape make geospatial technologies an obvious choice for capturing, storing, analyzing, and representing those phenomena. However, quantifying the past and digitally recreating and abstracting the landscape and its materials, raises questions as to how to best engage the cultural aspects of landscape within GIS. Additionally, questions arise concerning how to interact with and utilize qualitative information once it is in digital form to support spatial analysis (Cope and Elwood 2009). It is these discussions that evolved from, and eventually led to, the conceptualization of GIScience and guided the role of GIS and related spatial technologies in cultural geography research.

More recently, informed by discourse and developments in GIScience, researchers have had some success in integrating qualitative information within GIS (Cope and Elwood 2009). The social critique of GIS, early labeled 'GIS and Society' and subsequently 'Critical GIS', and research into Participatory GIS, have identified several perceptive insights into the social construction of GIS and to the biases and knowledge distortion that can occur unintentionally through the use of GIS (Pickles 1995; Craig et al. 2002; Kwan and Knigge 2006). Among many themes raised by GIScience was the need to incorporate qualitative information with more traditional quantitative spatial data (Elwood 2006, Harris 2016). 
Attempts to incorporate qualitative information within a GIS workflow have taken different forms from the incorporation of multimedia (Harris et al. 2000) to mapping locations in literary texts (Cooper and Gregory 2011). However, the goal of incorporating qualitative information into GIS analysis and representation has been problematic due to the nature of the data structures and analytical tools within current GIS applications. The potential for researchers to move GIS workflows beyond the generally accepted positivistic frameworks and to utilize a broader set of epistemologies is a defining feature of many of these approaches (Burgess and Orford 2010). Within these approaches, several potential avenues for incorporating qualitative information into GIS are offered, largely through a mixed methods approach (Cope and Elwood 2009). These approaches include the use of metadata to imbue qualitative information (Schuurman 2009), computer-aided qualitative analysis software (Jung 2009), and the use of visual geographies for representing qualitative information (Aitken and Craine 2009) among others. This mixed methods approach is similar to how anthropologists and sociologists are addressing the issues of incorporating computers and digital data within their own qualitative analysis (Lock 2003; Solomon 1993; Wheatley and Gillings 2002).

It is Kwan and Aitken (2010), however, that make the flamboyant statement that GIS are qualitative methods, and who suggest four critical ideas they feel pull GIS and qualitative research together including: qualitative methods and GIS, GIS as a qualitative method, qualitative visual methods and GIS, and community-integrated approaches. Each of these ideas describes how Geographers have, in fact, already brought GIS and qualitative research together. While many of the examples are not indicative of a 'tight-coupling' resulting in a definitive Qualitative GIS, they do show how GIS has been used to include qualitative information and qualitative research to date. 
A recent thematic issue of the Journal of Archaeological Method and Theory sought to address some of the issues related to the use of both GIS and phenomenology in landscape archaeology (McEwan and Millican 2012). The discussion on the Middle Ground between the quantitative nature of GIS and the qualitative focus of phenomenology offered thoughts and examples of epistemological and methodological approaches to intertwine these seemingly disparate ways of looking at the world. One paper even asked whether it was worth the time to attempt to link the experiential with the modeled as has been attempted to date (Gillings 2012). However, for the most part the authors offered a combination of methods to approach the issue. It was clear, though, that the authors and editors did not see a single approach to address this important aspect of the quantitative/qualitative debate, but a mixed methods approach from which to move toward a confluence of ideas.

GIScience research continues to build on mixed methods approaches to support the inclusion of qualitative information. Boschmann and Cubbon (2014) looked at the role that sketch maps can play in spatial data collection and conveying the participants' knowledge of the context being considered. Their focus centered on the individual nature of the data collected and its role within a qualitative GIS. Preston and Wilson (2014) look at what qualitative GIS and mixed methods can offer projects. They discuss the breadth of data collection, the depth of representations within the data, the iterative nature of their approach, the visualization of project information, and the process of their research approach itself. This discussion suggests that a mixed methods approach offers flexibility to address some of the 'epistemological uniformity' that many see as a result of GIS (Preston and Wilson 2014). Each of these papers offer an example of the trend in GIScience toward linking quantitative data and qualitative information to create a robust end product. 


\section{The role of GIS in landscape archaeology modeling}

At the base of any GIS or archaeology project is the insatiable need for data. In studying past cultural landscapes, it is the role of archaeology to provide data regarding the locations of habitations, raw materials, symbolic or sacred features in the landscape, work areas, refuse deposits, and standalone finds. Each piece of data that is available for a given area, whether material object or extant feature, helps create a more fully realized model or interpretation of a prehistoric landscape. At first, the role of GIS in archaeology (as was common for most GIS applications) was that of a data repository, a database for archaeological data. GIS, however, provides much more than database functionality through its visualization and cartographic tools, data analysis tools and algorithms, and through its query functionality. By allowing several sources of data to be viewed concurrently and in an exploratory manner, new questions often arise about spatiality and relationships that are not seen in disconnected data sets.

A strong focus on data has thus come to the fore and is essential in the use of GIS as new technologies, including sensors (physical and human), storage capabilities, and presentation methods, are driven from within the myriad professional and user-driven endeavors built on geospatial technologies. Ever increasing sensor resolution, especially digital sensors, have made aerial and satellite data integral to data collection and has moved GIS from an early focus on digitizing data from existing maps to capturing contemporary geographies by remotely sensed imagery. New elevation data captured from high resolution stereo pairs, synthetic aperture radar, and Lidar have made the analysis of surfaces and intervisibility more precise (Rouse 2004a). As computing power has reached a point where complex GIS analyses take minutes as opposed to hours to complete, and data storage has expanded to allow users to store large quantities of data locally or in the cloud, so the computational and data constraints of GIS have largely been 
overcome. The internet has also had a significant impact on presenting and sharing not only data through clearinghouses, but also through web data services and the sharing of information through web-based map distribution, both static and dynamic (Rouse et al. 2007).

Geospatial technologies have been used in archaeology for decades beginning with the use of aerial photographs to search for, and map, archaeological sites and features. Archaeology's data collection is tightly bound to the spatial measurement of sites and excavations, and often extends beyond traditional 2D surface mapping into the third dimension as extant features and objects are measured below the surface. Over the last twenty years archaeologists and GIScientists have outlined how culturally relevant data from archaeological surveys and excavations can be used in a multitude of ways (Allen et. al. 1990, Lock and Stančič 1995, Lock 2000, Wescott and Brandon 2000, Wheatley and Gillings 2002).

From predictive models to intrasite analysis, GIS has been adapted to support archaeological investigations with a mix of success and disappointment. While the successes are readily attributable to the spatial nature of most archaeological information, the disappointments are often related to a lack of understanding of the issues, technical and methodological, that are inherent to GIS. Lock and Molyneaux's (2008) text, for example, focuses on one of the most overlooked issues in dealing with spatial information, the impact of scale. Scale related issues such as the Modifiable Areal Unit Problem, ecological fallacy, or others must be understood as they impact the use of spatial data (Harris 2008). This is not to suggest that GIS and the data used in a GIS are unavoidably flawed. Instead it is to suggest that users of any spatial methodology, technique, technology, or tool must first understand the strengths and limitations of their chosen approach in order to identify and achieve optimal results and interpretation. 
In the case of landscapes, or similar cultural phenomena, GIS has struggled with qualitative data and with adapting to the many contemporary theoretical developments in human and cultural geography. This constraint certainly applies to phenomenology and experiential approaches. Whether qualitative information is from field data such as photos and field notes, or associated research such as a literature review of local sites, it is possible to store the information, but GIScientists are still working on approaches to the spatial analysis of text, images, audio, and other qualitative data sources using GIS applications (Bodenhamer et al. 2010). It is possible to address this issue through the loose coupling of qualitative analysis with GIS, but this remains a kludge at best. New innovative approaches are required to ensure that GIS can fulfill the role of an information system capable of addressing a mix of spatial and multimedia data analysis from a variety of theoretical perspectives beyond that of positivism. 


\section{Chapter 3 - Perception and Visual Landscapes}

Phenomenology and GIScience at first glance appear to sit at opposite ends of the postmodern/positivist spectrum. GIScience is embedded in a positivist mode based on scientific enquiry and methodologies tied to empirical observation and the need for replicable results. Positivism is premised on hypothesis testing and framing research questions that can be tested through the collection of data and the execution of models to determine the observed outcome. The goal in this case is to create or support a series of unifying and overarching principles and to build a shared understanding of a phenomena or situation. Conversely, phenomenology is framed as individual, subjective, and qualitative, and focuses on the premise of experience.

Phenomenology has deeper roots, however, which are not as distanced from scientific inquiry as this simple comparison would suggest. Phenomenology, as an ontology, is based on reflection and the description of experience. What is recorded in the initial phenomenological description are the object(s) or phenomena as experienced through the subjective lens of the investigator's consciousness, intentions, and personal background to experience and evaluate the phenomena. As Relph (1970: 198) suggested:

"From the basis of these phenomenological assumptions attempts to develop mathematical models and theories of man's behaviour in space are seen not as a contribution to an understanding of some "real" geography of man's activities, but as the reflection of the limited intentions of those geographers presenting the explanation." 
Attempts to model the world in GIS have traditionally been intended to answer a spatial question or test a hypothesis, and not necessarily to explore an interpretation of a cultural site, activity, or landscape. This is not to suggest that models are limited in how they can be used, but that they have been used in a limited way due to the nature of quantitative techniques.

However, like GIScience, phenomenology does not exist to the exclusion of other conceptual methods, and phenomenology is in fact arguably best utilized within a broad-based approach. To tightly couple Phenomenology and GIScience, in pursuit of a cognitive experience of landscape, some common themes must be found that can take advantage of both traditions. Perhaps the most likely linkage between a phenomenological view of the world and the way in which space is conceptualized in GIS is through the visual and sensory response to landscape. The importance of the senses in a phenomenological approach cannot be overstated. As suggested by Merleau-Ponty (2002), the body acts as a conduit between the world and human consciousness through the five senses: auditory (sound), olfactory (smell), gustatory (taste), haptic (touch), and optic (vision). The senses act as translators to convey perceived objects to the consciousness. While humans are awash in information from our various senses, for many the sense of sight dominates.

Rock (1995) places existing perspectives of perception into four groups: 1) The Inference and Empiricist Perspective, 2) The Gestalt Perspective, 3) The Stimulus Perspective and 4) The Information Processing Perspective. Rock's Inference and Empiricist Perspective is based on the work of $18^{\text {th }}$ century empirical philosophers who argued that the ability to perceive the world is built on sensory experience. They argued that what was perceived directly was inadequate to fully understand the world and that it was necessary to learn how to interpret perceptual cues. Understanding the world, they reasoned, is heavily tied to the human capacity to associate 
perception with physiological cues such as the thickening of our ocular lenses or the shifting of facial muscles. Later in the $19^{\text {th }}$ century, Hermann von Helmhotz, who linked perception to a process of inference, developed these ideas further. Inference, he suggested, is based on past experiences through which "we infer from the sensations we receive at a given time the nature of the object or event that they reasonably represent." (Rock 1995: 9). This largely subconscious inference process builds on an individual's previous sensory experiences.

The Gestalt Perspective (Wade and Swanton 1991, Rock 1995) is driven by our tendency to view objects as a whole. The central point of Gestalt theory as it relates to the way in which we perceive the world is in the context of grouping. To comprehend complex information flows humans tend to interpret a visual field or problem in a way that organizes and unifies specific elements into groups of related objects. This perspective differs from the inference and empiricist perspective in that Gestalt perception is based on spontaneous interactions in the brain resulting from sensory input. There are four main factors that affect the construction of Gestalt groups (Rock 1995):

1) Proximity describes how elements are grouped depending on their spatial proximity to one another.

2) Similarity, whereby items that share some attribute tend to be grouped together.

3) Closure defines items that, as a group, complete a pattern.

4) Simplicity clusters items that are organized into figures according to symmetry, regularity, and/or smoothness. 
These four factors are limited by their implied assumptions. For instance, placing an actor in a landscape at a certain location will break the landscape into a particular set of groupings based on the aspects of proximity, similarity, closure, and simplicity that are observed. However, by simply moving the actor to another location in the landscape, these groupings will change as the aspects of each of the factors are altered based on the new location. The changes in these groupings should not have a significant impact on the understanding of the landscape as a whole and could indeed potentially broaden the knowledge of the whole by providing a holistic context for the objects in the landscape.

Perhaps the perspective that best correlates to a phenomenological ontology, at least as considered herein, is Rock's Stimulus Perspective. The Stimulus Perspective does not directly build on the ideas of the empiricist or on Gestalt perspectives that assume that the senses receive an inadequate amount of information to stand as the sole basis of our perception. Instead, the Stimulus Perspective suggests that all stimuli needed for perception exist in the environment and therefore "there is no need to postulate such mechanisms as unconscious inference or spontaneous neural interaction to explain perception.” (Rock 1995: 12). This psychophysical approach to perception was significantly extended by the work of J. J. Gibson and his students and contemporaries (Wade and Swanton 1991). A notable contribution of Gibson's work is the focus on the concept of affordance which is the potential held by an object for a particular set of actions (Dant 2005, Knappett 2005). A key aspect of affordance is that it is dependent on the interaction between both the perceiver and the environment (Wheatley and Gillings 2000). For instance, an earthen mound in the landscape offers different potentials or affordances based on the observer's cultural perspective. A modern hiker may see the potential of a mound as a feature to be climbed and used as a point of observation; an archaeologist may see the potential for 
scientific enquiry through excavation or survey; and a Native American may see the potential for the reverence of ancestors who built the mound. While the phenomenon in the environment is the same for each observer, the affordance ascribed by each observer may vary considerably based on the various presuppositions.

A final perceptual perspective, as it relates to issues aligning a phenomenological ontology with a GIScience methodology, is the Information Processing Perspective (Rock 1995). This perspective perceives stimuli as energy that is observed and subsequently processed into information. Beyond the cognitive processing of the Information Processing Perspective, there arises the question of how much information processing can be done outside of the human mind and yet continue to contribute to meaningful comprehension. In the processing of experience, sensory and stimulus overload can often create confusion. With the use of computing technology, the opportunity exists to parse some of the grouping and patterning of the Gestalt processing load to the computer where portions of this processing can be done by software through models or neural networks. Moving these demanding portions of the processing away from the human mind could enable ever greater amounts of information to be perceived and processed to support a broader perspective, and yet allow the human mind to create the necessary inferences and interpretations arising from that information.

It is no small stretch to connect each of Rock's four groups with research activities ranging from field work to interpretation. The inference perspective is heavily connected to the empirical approach and to data collection in the field. The Gestalt perspective dovetails with how we group data into 'layers' within GIS. As suggested, the stimulus perspective provides a description of the psychophysical approach taken by Gibson and others. Finally, the information processing perspective connects to the modeling and analytical capability of GIS. Each of these 
perspectives impacts the way in which we perceive and record the world around us to create data, information, and interpretations. It is the need to understand how information is perceived and interpreted that may best guide how the coupling of the ontological and methodological approaches proposed here are used to link GIS and phenomenology. While the role of experience is paramount, how we conceive and describe experience through text, photos, audio, or other methods all contribute to how we interpret the landscape.

\section{Perceived landscapes}

Gibson's (1950) psychophysical conceptual framework has been used by many researchers to support very detailed investigations of human perception and interaction with landscape objects (Higuchi 1988, Ingold 1993, Cosgrove 2008). However, the surface has only been scratched by geographers in this area of investigation and, for the most part, only the simplest evaluative metrics have been utilized in the exploration of landscapes. For example, the discussion of viewsheds, intervisibility, viewscapes and other measurable ways of discerning and modeling landscapes rarely go beyond simple measured or modeled visual metrics. This is of some concern especially since many users of geospatial technologies are guided so heavily in their work by the tools made available to them rather than by incorporating appropriate methodologies and principles that underlie them.

Some of the early discussions of Cultural Geography by Sauer (1925) assigned a level of importance to the experience of landscapes. This has continued through more recent work by Relph, (1970), Tuan (1974), Muir (1981), Seamon and Mugerauer (1985), Ingold (1993), and Tilley $(1994,2010)$ to name just a few. However, of these studies, Appleton's (1975) The Experience of Landscape offers, perhaps, the best example of how visual perception impacts our 
experience and interaction with landscapes. Appleton proposed that humans view the world through vistas, "a view which is restricted by conspicuous bounding margins" (1975:85), and vantage points, the position of an observer in a landscape. From these two concepts Appleton draws on a wide range of arts from painting to literature to assess how they represent what he calls prospect-refuge theory. Prospect-refuge theory is based on two perspectives of a given landscape, whether it is "an unimpeded opportunity to see", a prospect, or "an opportunity to hide", a refuge (Appleton 1975:73). Appleton sought to go beyond the simple measurable description of what can, or cannot, be seen and to build a compelling argument of how we as humans perceive and experience landscapes, not only physiologically but atavistically. It becomes clear from Appleton's work that the perception of landscape is both personal and qualitative in that experience connects the observer and their actions to the landscape and in so doing provides an evaluation of the meaning of the landscape to the observer. These concepts go well beyond the measured viewshed analysis of GIS and contribute considerably more to landscape assessment and evaluation.

\section{GIS and the modeling of landscapes}

There are several spatial models used in evaluating visual landscapes including isovists, viewsheds, and viewscapes. Each of these models offer a method, or methods, for capturing different visual metrics of the landscape. Isovists according to Benedikt (1979: 48) are "locationspecific patterns of visibility". While the primary use of isovists has been for studying architectural space (Batty 2001, Turner et al. 2001), some research takes isovists beyond the literal measurement of urban vistas to examine human psychological links to landscape in general (Wiener and Franz 2005). Benedikt (1979), for example, used isovists to consider object 
perception, and by focusing on a specific feature and a broader environment perception sought to encompass specific objects within that broad environment. This expansive view, harkening back to Gibson's work on visual perception, allows for a visual perspective of landscape that extends beyond the component parts, or objects, in an attempt to consider the visual impact of the environment as a whole. The methods for modeling isovists are relatively uniform and are based on the initial work outlined by Benedikt (1979) and in the related associated research reported by Davis and Benedikt (1979).

Perhaps the most common method for the visual modeling of landscape using geospatial technologies is what is generally referred to as a viewshed. A viewshed represents a portion of the earth's surface that can be seen from a particular location on the topographic surface (DeMers 1997). The results of a viewshed model rely on three parts: 1) an interpretive algorithm, 2) a terrain model, and 3) a definition of the viewpoint of the observer. The algorithms used to model viewsheds determine what areas of the terrain are occluded due to topographic obstructions. While the primary differences between existing viewshed algorithms tends to be the computing run-time needed to process the results, there can be variations in results between models as a result of the different algorithms (Fisher 1996).

The resolution, accuracy, and type of terrain model and data used in conjunction with a viewshed algorithm play a significant role in the modeling of viewsheds due to the unique characteristics and limitations of each available dataset (Riggs and Dean 2007). The ideal terrain model would reflect a true 1:1 fidelity with the real world. However, for obvious reasons it is necessary to abstract the terrain model to different levels of resolution. Variations in model results can be tied to the myriad resolutions and storage methods (TIN, raster, vector) and the algorithms used to model the terrain. These variations can result in landscape features being 
overrepresented or underrepresented, and data artifacts or errors being introduced that impact the efficacy of the modeled results (Christopherson et al. 1991). Within the landscape itself, features can either obstruct a view or open a view. An analogy of this is the 'haha wall' which allows for an unobstructed view from one vantage point but appears simply as a wall from the opposing direction (Appleton 1975). The purpose of these walls is to allow for open vistas while creating a physical barrier to animal access. Because haha walls are generally low in height it is likely that in most terrain datasets they would not be represented in a viewshed analysis due to the smoothing of the landscape through low resolution terrain models.

Terrain data can play an important role in influencing the quality of the viewshed. Modern remotely sensed data, depending on its method of data capture, can include vegetation height, denuded bare-earth surface, or some combination of the two. The presence or absence of vegetation has been shown to have a significant impact on viewshed results (Harris et al. 2000). Most viewshed models enable the location and viewing height (offset) of the observer or viewpoint to be specified along with other attributes. Attributes such as the viewpoint height above the surface allows for the consideration of differing vantage points. Defining the direction and angle of view will also have an impact on the vista. These attributes all influence the resulting viewshed model.

Finally, viewscapes provide a visual modeling method whereby areas act as a unit based on the connectedness of the visual elements within the vista (Rio Blanco 2002). Canada's Historic Places (2010) defines a viewscape based on line-of-sight from a specific location to the landscape in which it sits. Overall, a viewscape is a less used concept and is primarily utilized to denote the study of a view connected to specific policy considerations. The Rio Blanco County Land Use Resolution (Rio Blanco 2002: 1), for instance, suggests that: 
"[v]iewscapes are defined as those features that provide a range of sight that can be identified as providing a community asset such as but not limited to pleasing vistas, scenes and view that provide a sense of place and character. Views within viewscapes include pastoral open space vistas, skylines, ridgelines and peaks. These aspects provide an important sense of place and need to be considered as development that impacts these features can have a profound economic and social impact....”

While the use of the term viewscape is dominated in connection to landscape and land use policy, some researchers have also adopted the term to refer to a more generalized visual investigation of a landscape. Wilson et al. (2008:109-110) used the phrase biophysical viewscape modeling because they felt it allowed them to integrate "biological and physical approaches of environmental remote sensing with viewshed analysis to estimate components of landscape perception." Canada's Historic Places (2010) differentiates viewscape from viewshed in suggesting that a "viewshed refers to a sequence of views or panorama from a given vantage point" whereas a viewscape has a broader perspective than the specific vantage points of the viewsheds.

Overall, the three concepts discussed, isovist, viewshed, and viewscape, form the basis of most approaches to modeling the visual qualities of landscapes. They provide a way to represent the visual perception of landscape, to model the results of an embedded experience of a landscape. These approaches share a clear connection with the vantage point and vistas discussed by Appleton and can provide insight into cognitive responses such as prospect and refuge. But at 
the same time the models alone do not provide a cultural perspective or suggest a descriptive or interpretive methodology to take the modeled data further.

While Appleton discussed the observer/observed relationship through vistas and vantage points, the subsequent shift to GIS has disconnected the relationship between viewshed and observer and removed the cultural or social importance of the act of viewing and meaning from the viewed assessment. In the last two decades, the role of landscape evaluation as modeled through GIS has arguably limited the interpretation of landscape. Work in geography, landscape architecture, and landscape archaeology have shown how geospatial technologies can be used to capture what the human eye sees without having to physically visit a specific location in space or time. These technologies offer simplistic 'one-click' results to significant questions based on fixed algorithms, such as the extent of visible area from a given point in the landscape. This reductionist approach to landscape viewsheds has made these tools enticing to GIS users and landscape researchers. However, the simplicity of these models clearly leaves many aspects of the landscape unconsidered. 


\section{Chapter 4 - Combinatorial methods for exploring 'scapes}

Archaeological inquiry into prehistoric landscapes has been limited due to the obvious lack of direct observation, data, and testing. However, ethnoarchaeology, ethnography, and knowledge of human/environment interaction have made it possible to postulate and even simulate scenarios concerning activities that took place within prehistoric cultural landscapes. It is the transition from landscape observation to landscape representation through the confluence of experiential approaches and spatial modeling that lies at the core of this study. The discussion of Middle Ground approaches (McEwan and Millican 2012), that link quantitative and qualitative techniques, continues to garner attention in the midst of ever more refined approaches to data capture, data access, and data analysis. The issue remains, however, as to the analysis and interpretation of an archaic culture.

Aspects of archaeological landscape activities have been modeled using a variety of methods including resourcescapes (Trifkovic ND), taskscapes (Ingold 1993), and visualscapes (Llobera 2003). These 'scapes are potentially powerful concepts if brought to bear in addressing the landscape. Each of these 'scapes is tied to examining how people interact with the objects or features within a landscape. For example, in order to create a tool such as a pot, there are certain materials that are required. The potter must have an appropriate source of clay, a tempering agent, wood to build a fire, materials for decoration, and so on. Each resource must be identified and acquired before the potter can complete the vessel. While the specific cognitive processes of the potter tied to their use of the landscape cannot be directly modeled, the location of potential materials can be relatively accurately mapped. From the locations of the resource materials it is possible to consider the optimal access paths, the best material sources, or the travel routes that 
served additional needs such as foraging, that the potter could undertake. This example of a 'resourcescape' is somewhat simplistic, but it does show the potential of integrating archaeology's focus on material culture with the spatial modeling capabilities of GIS in exploring a landscape.

In defining the concept of 'taskscape', Ingold (1993: 158) states that tasks are the constitutive acts of $d$ welling and activities that are conducted within a social context of life. A taskscape is defined as an array of related tasks and activity spaces and a sequence of acts that are conducted individually or by a group as part of what Ingold calls 'dwelling'. These taskscapes can be specific or broad and encompass a range of activities from daily tasks such as hunting or gathering, to sacred tasks such as burial or mound building.

The role of spatial analysis in understanding the movement associated with tasks has been outlined in texts such as The Early Mesoamerican Village (Flannery 1976a, Flannery 1976b). Flannery examined the role of site catchment in assessing how far villagers were likely to travel for various activities. Flannery determined that the agricultural needs of a village could be supported within a distance of 2.5 kilometers $(\mathrm{km})$ of an early mesoamerican village, while the area within $5 \mathrm{~km}$ could support most of its mineral resource needs. The range for hunters could have extended as far as $15 \mathrm{~km}$ from a village. In an exploratory approach that questioned how far materials had been transported to a given village, specific quarries and similar material sites that were further away were invariably connected to other tasks that were not conducted on a daily basis such as knapping and pottery making. Flannery and others have been able to identify the source of material remains uncovered through excavation to determine the nature of the physical environment from which they originated and thereby identify areas that were likely used in conducting various village activities. In essence, they were able to delineate taskscapes. 
An example of a taskscape could be the creation of shell mounds. In the building process of a shell mound a known material (i.e. shellfish) is required that has limited availability and a connection to a specific landscape feature, that of water. Over time, as shellfish are harvested and processed, and the mussel removed, the shells are invariably deposited in a single location and the mound grows. There are different methods of deposit that create mounds of different sizes and shapes (generally based on regional or temporal variations), but the taskscape remains mostly the same: collect shellfish from the water feature (river, ocean, lake), take them to the processing area (to be smoked open, cracked), and pile the remaining shells in a designated area.

In the same way that taskscapes can be identified by the activities that take place within them, so might 'visualscapes' provide an appropriate model for the visual perception of cultural landscapes. Llobera (2003:30) defines a visualscape as "the spatial representation of any visual property generated by, or associated with, a spatial configuration." Just as there are different types of landscapes based on culture, physical features, or task, so visualscapes may vary by landscape properties. Llobera (2003) focused on three visual properties of landscape in his discussion of visualscapes: cumulative (or total) viewsheds; visual prominence; and visual exposure. Cumulative viewsheds determine areas commonly visible from different locations in a landscape (Wheatley 1995) and are the result of viewshed analyses from multiple points of observation. Visual prominence is a measure of how conspicuous a given feature is in its surroundings. Visually prominent features could be deliberate acts as territorial landmarks or navigational markers within a landscape. Visual exposure is a description of "how much of a feature or a terrain is visible at each location..." (Llobera 2003: 40). Llobera's discussion of visual angles as a component of measuring visual exposure is centered on the angle of view of an object or feature from a specific position. For example, from a distance, the viewshed of a 10m 


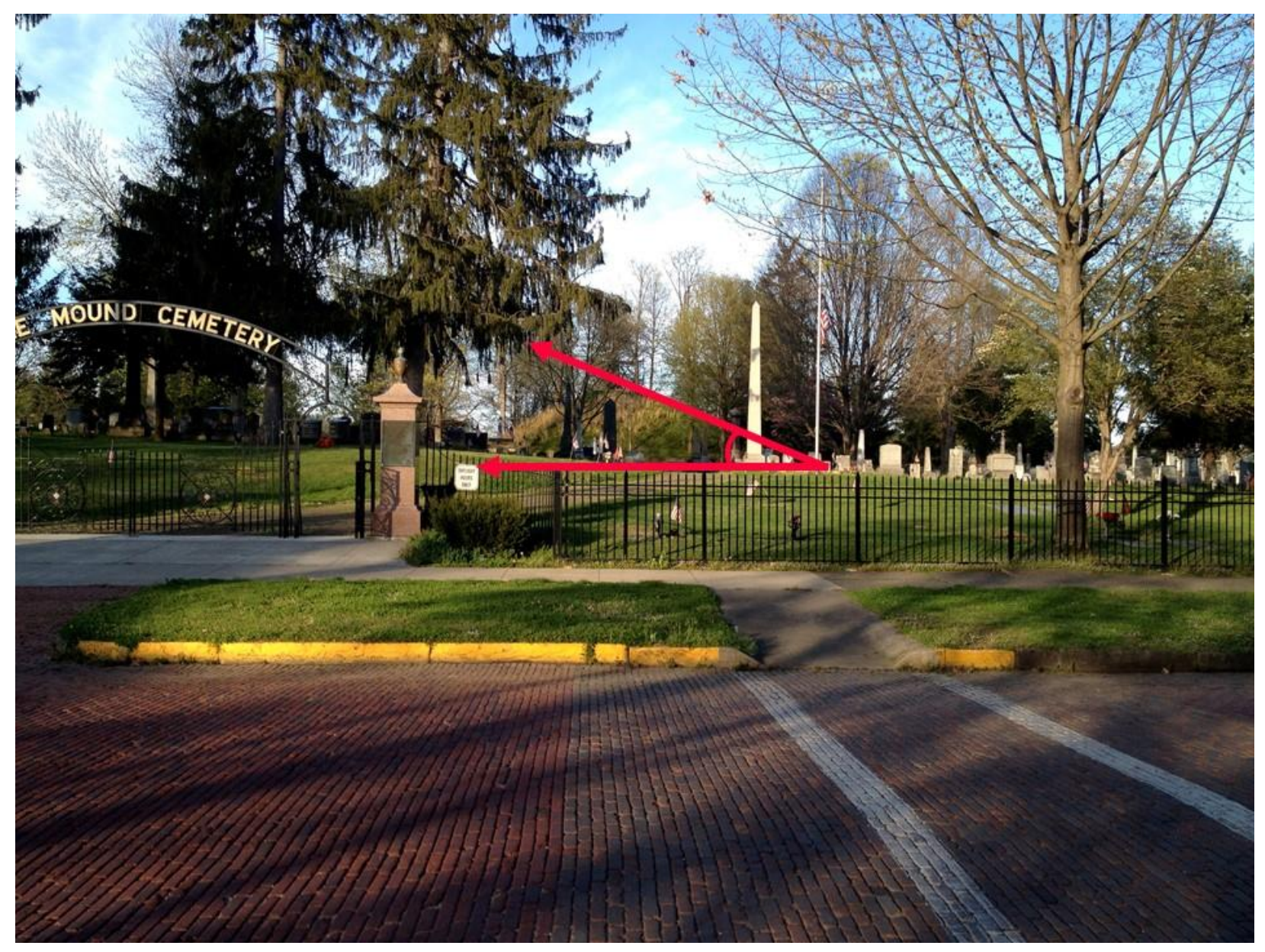

Figure 4.1. Small view angle of a large object from a distance

tall earthen mound may be minimal and the angle from a viewer's point of reference to the apex

of the mound would be similarly limited (Figure 4.1). However, the mound viewshed and the viewable area when standing near the base would be significant as would the angle from the viewer's point of reference to apex of the mound (Figure 4.2). The ability to capture such a personal perspective as a view and to utilize it to understand cultural attachment to, or delineation of, a landscape is exciting.

Resourcescapes differ from the cognitive base of taskscapes and visualscapes. Instead, resourcescapes are centered on a physical geocentric perspective of the observed or recorded 


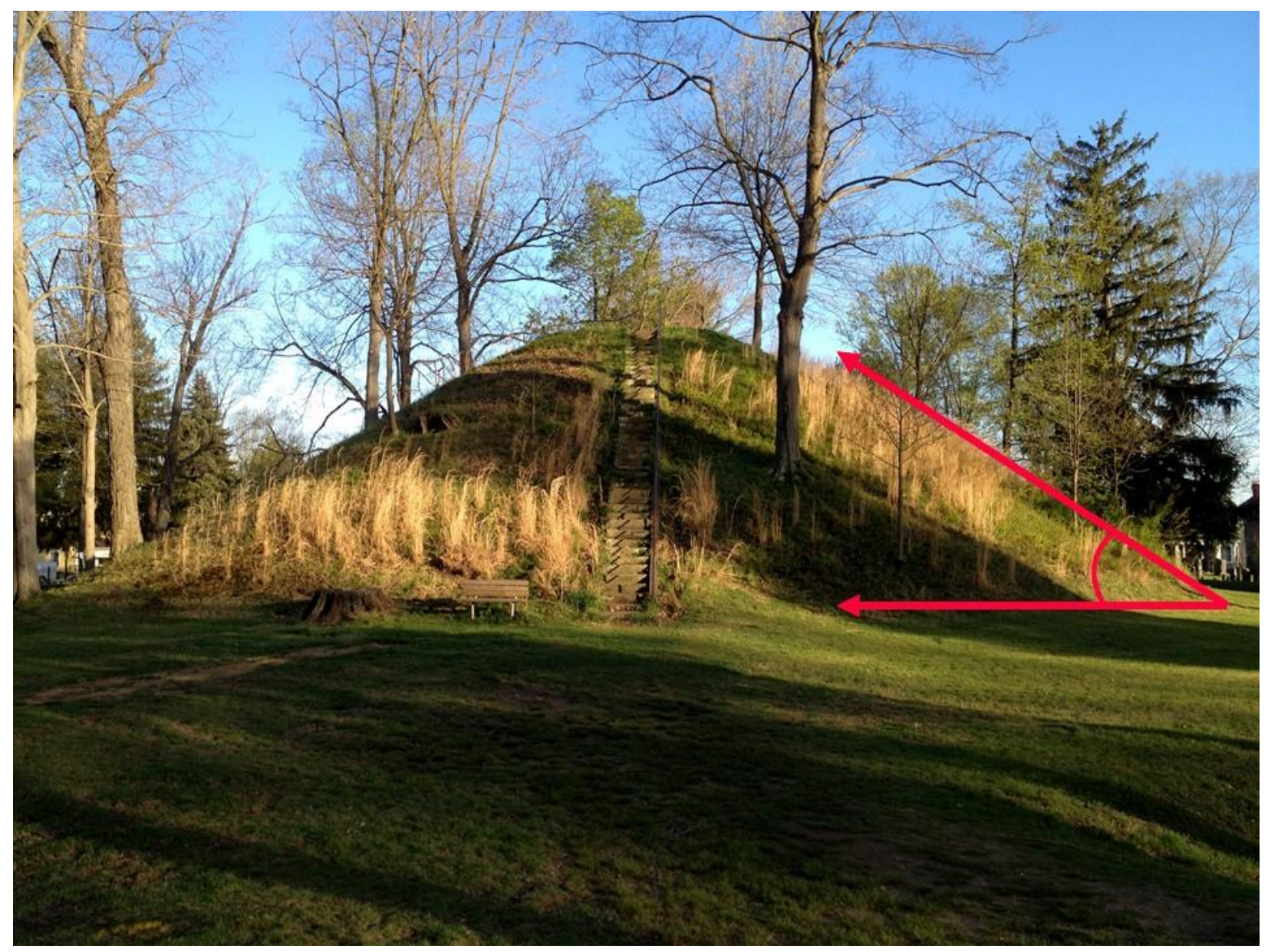

Figure 4.2. Large view angle of an object from near the feature

characteristic of known materials or resources obtained from archaeological excavation and finds. Whether raw materials or finished tools, these resources can be used to identify humanenvironment relationships within a cultural landscape. The earlier pottery example focused on the acquisition of material resources needed to construct a ceramic vessel. In a resourcescape approach, materials are considered separately from their intended use in the task of construction and are instead considered in the context of how the resource locations are interrelated. Another example could be resources that were traded into a region. The Hopewell culture, for instance, had exceptional trade networks and resourcescapes where tools and ornaments made from conch 
shells that originated from the Gulf of Mexico and mica from North Carolina, along with other exotic materials, have been found in sites some distance from their source location (Struever 1977, Goad 1979). Each of these items has a source that may or may not be related to a specific location, however, a map of the relationships between the materials' origin(s) and the excavation location can be offered (Figure 4.3). Each of these material examples show that resources often came from outside the taskscape in which the tools or ornaments were likely fashioned

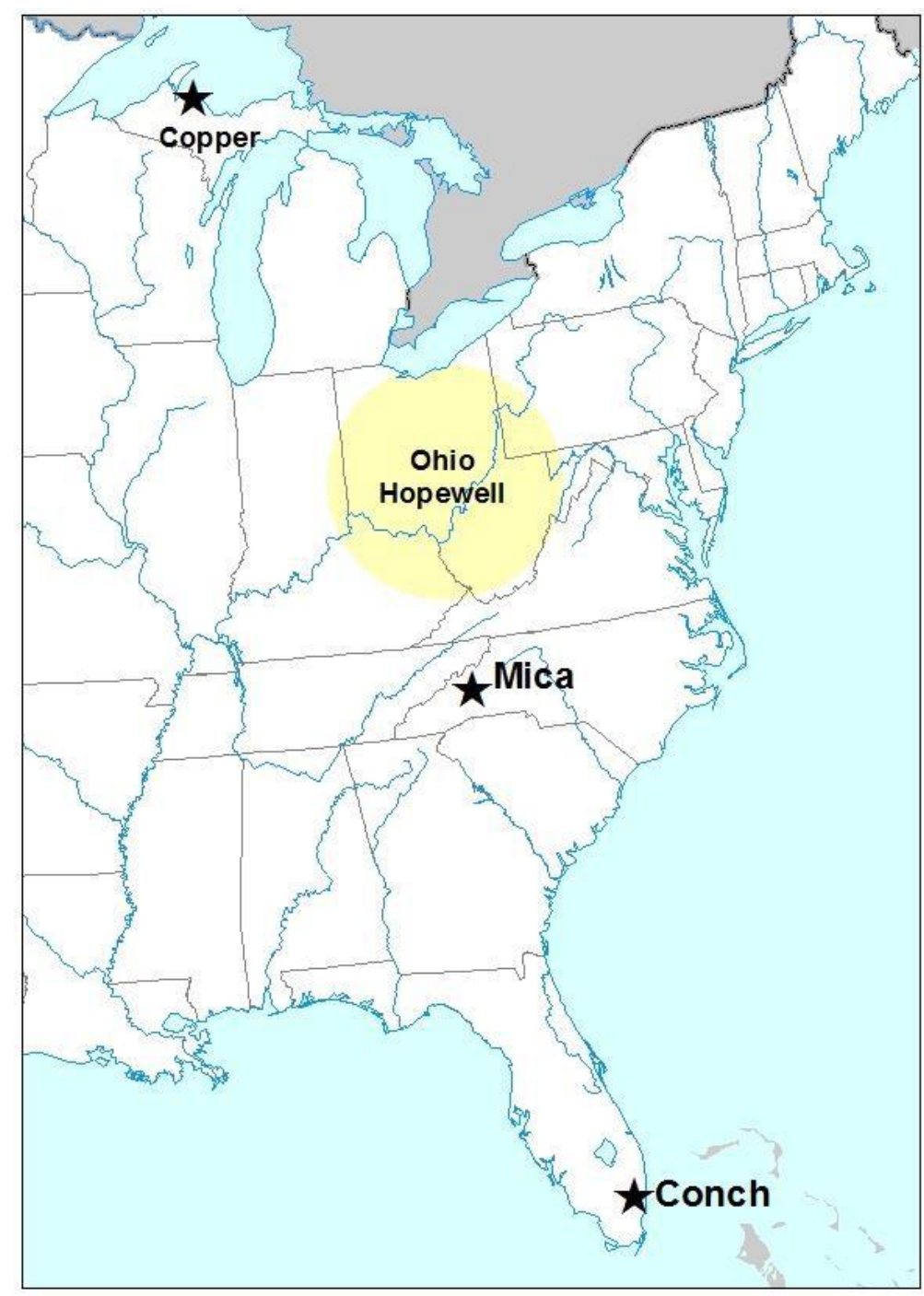

Figure 4.3. Material sources within the Hopewellian Interaction Sphere and that their origins could have been remote from the cultural landscape in which the final artifacts were used. A resourcescape then, is a landscape defined by the relationship between recovered artifacts or features, the areas from which the raw materials originated, and the places where the products were eventually used (Epstein and Axtell 1996).

Importantly, taskscapes and visualscapes have significant linkages to the prehistoric 'individual' as both tasks and views originate from the human actor in the landscape. A 
resourcescape is primarily a geographic and generalized concept that does not focus explicitly on the human mechanism, but instead is premised on the spatial locations and relationships of the materials. By modeling the specific connections to a landscape such as the tasks conducted or the visible landscape, valuable insight can be derived to support an interpretation of a cultural landscape. Considering specific 'scapes within the context of a holistic model of landscape, new insights might be unearthed regarding past cultures.

\section{Implementing 'scapes}

Attempts to operationalize phenomenology in landscape archaeology have focused primarily on in-situ personal experience through concepts such as Heidegger's daesin, or 'being in the world'. These individualistic, personal approaches to experiencing landscape fit well within a postmodern approach, but arguably lack the rigor sought by the social sciences. Tilley (2004: 219) states that a "strong gut reaction by some to a phenomenological approach is that one must be able to verify experience in some way." However, it can be argued that verification alone cannot be a singular goal of any interpretive endeavor. The evaluative nature of the human experience often yields a unique response tied not only to the human condition, but to the changes that are inherent through the progression of time. Assessing a landscape through a structured set of combinatorial and perceptual indices as proposed in this dissertation has the potential to contribute to a blending of the individualistic experience in phenomenological archaeology with the observation and rigor of GIS. In so doing, it might be possible to move beyond the processual/positivist stovepipes that have remained in place since the 1970s and provide a means for researchers to share, and as importantly, compare information based on a common foundation while accepting the individual's perspective. 
It is clear that when viewing a landscape to gain insight into its past, the ability to bring information that extends beyond the extant landscape would usually be beneficial. For instance, the ability to extract meaning from existing historical descriptions or archaeological research can provide unique insight into a landscape regarding features or phenomena that may have been refashioned by cultures that subsequently lived in the landscape. And while it may be optimistic to conceive and describe a landscape as a whole, the use of geographic information can provide unique spatial representations of particular aspects and time slices of the landscape.

Hodder (2003) describes contextual meaning through two lenses. The first is that of the environmental and behavioral context of action. Landscape provides both the 'larger functioning whole' with which it is possible to gain understanding of an object within a landscape and simultaneously landscape can be considered as the object that needs to be understood in a larger contextual analysis. The second meaning is "with-text", or an interpretation associated with the surroundings. If an object is a word and a landscape a chapter to be interpreted, GIS can act as the modern illuminated volume within which the (con)text is copied and viewed. The use of the ontological approach of phenomenology and the data driven technology of GIS come together, within a closer relationship, through the use of context and through Hodder's framework for contextual archaeology.

Once an observer has created a context for a landscape, their experience of landscape is built on perceptions conveyed through descriptions of that landscape. However, without some foreknowledge of a landscape and its contextual elements the research of past landscapes would be highly constrained. As Tilley (1994: 67) suggests: 
"The landscape is continually being encultured, bringing things into meaning as part of a symbolic process by which human consciousness makes the physical reality of the natural environment into an intelligible and socialized form.... Histories, discourses and ideologies are created and re-created through reference to the special affinity people have with an area of land, its topography, waters, rocks, locales, paths and boundaries."

Personally viewing an area raises several critical points concerning landscape perception and especially the experience of landscape. Experience is not just a viewshed, or what can be seen or is occluded, but the effect of an individual's perception of distance on what can be seen and meaningfully discerned, the color and texture of features, whether looking ahead or up or down impacts the experience of the landscape. All of the factors that humans experience when viewing the landscape alter the perception, and therefore the experience, of the landscape incorporating the role of the human agent in blending context, history, and culture with what can be seen. Higuchi's (1988) work provides an intriguing framework for linking our visual perception to landscape assessment and thereby aiding a phenomenological and experiential perspective of archaic landscapes. 


\section{Chapter 5 - The Higuchi viewshed: from measured view to measured experience}

The indices outlined by landscape architect Tadahiko Higuchi in The Visual and Spatial Structure of Landscapes (1988) provide an intriguing method for inserting the human into the visualscape and for assessing the perceived quality and experience of landscape. Higuchi provides an innovative approach to viewshed assessment that takes into account not only the nature of the physical landscape, but also the physiological and perceptual responses of the human body and mind in viewing and responding to that landscape. As such, Higuchi viewsheds may provide a critical bridge between the quantitative measurement of GIS and the phenomenological experience of landscape.

Integrating both egocentric and geocentric perspectives of the world is key to the approach proposed here. An egocentric perspective is clearly related to Tilley's and Higuchi's work, based as they are on the experience of the "now," and on the observer's immediate location in the landscape as well as their physiological and cognitive responses to a landscape. The geocentric perspective is tied more to the broader spatial, cultural, physical, and archaeological elements of a landscape. Geospatial technologies provide a platform that allows the geocentric world to be readily captured and modeled. However, it is argued here that bridging the egocentric, privileged by the experiential, with the geocentric, utilizing the scientific knowledge of a landscape, acts to reinforce phenomenological landscape archaeology. In essence, it is the merging of the modeled geocentric perspective with the egocentric, experiential perspective that contributes to a richer experience of landscape and enhances understanding of the potential immaterial, as well as material, symbolism within a cultural landscape. This perspective differs 
from most conceptualizations of Qualitative GIS where qualitative information is used to support the technical analysis. Instead, combining geocentric and egocentric views of the landscape allows for the quantified aspects of geospatial technologies to augment and complement a user's egocentric experience of a landscape.

\section{The Higuchi Viewshed}

The term Higuchi viewshed is somewhat deceiving as, in contrast to traditional viewshed assessment that is predominantly based on line-of-sight, Higuchi viewsheds represent a significantly more sophisticated and complex assessment of landscape quality. In scope, they are more viewscape than viewshed and in modeling terms they extend well beyond the isovist approach. Higuchi sought to incorporate elements of human perception and human behavior within indices and, by so doing, embed the human experience into landscape quality assessment. Based on human physiological and psychophysical characteristics, Higuchi's indices seek to encapsulate both the human perception and the visual aesthetics of landscape to contribute to human centered experience of landscape. While a reading of Higuchi's original text is highly recommended for the details provided, a short overview of his indices is provided here.

Higuchi (1988) outlined eight distinct indices comprising: Area of Visibility, Distance, Angle of Incidence, Depth of Invisibility, Angle of Depression, Angle of Elevation, Depth, and Light. Each index provides valuable information on its own, but when combined they provide deep insight into a landscape and its characterization. The role of each index is important in understanding the overall intent and methodology that Higuchi laid out.

Perhaps the most dominant index in terms of determining what lies within an individual's field of view is the traditional measure of visibility (or invisibility). The visibility index, as with a 
traditional viewshed analysis, delineates the area that can be seen from a particular location or multiple locations. The key instrument throughout Higuchi's indices is the human eye, but in Higuchi's analysis the area delineated by visibility yields a more subjective measure. Visibility and intervisibility are heavily influenced by the location of the observer and the characteristics of the human eye. If the visibility is based on someone standing on the rim of the Grand Canyon, they will likely have a significant vista. If, however, the viewpoint is based on someone standing in a forest, then the area will be significantly constrained and the viewshed likely measured in terms of a few meters. In both of these examples there will be areas of invisibility where objects in the landscape may be present but are not visible. A rock mound might be hidden behind a

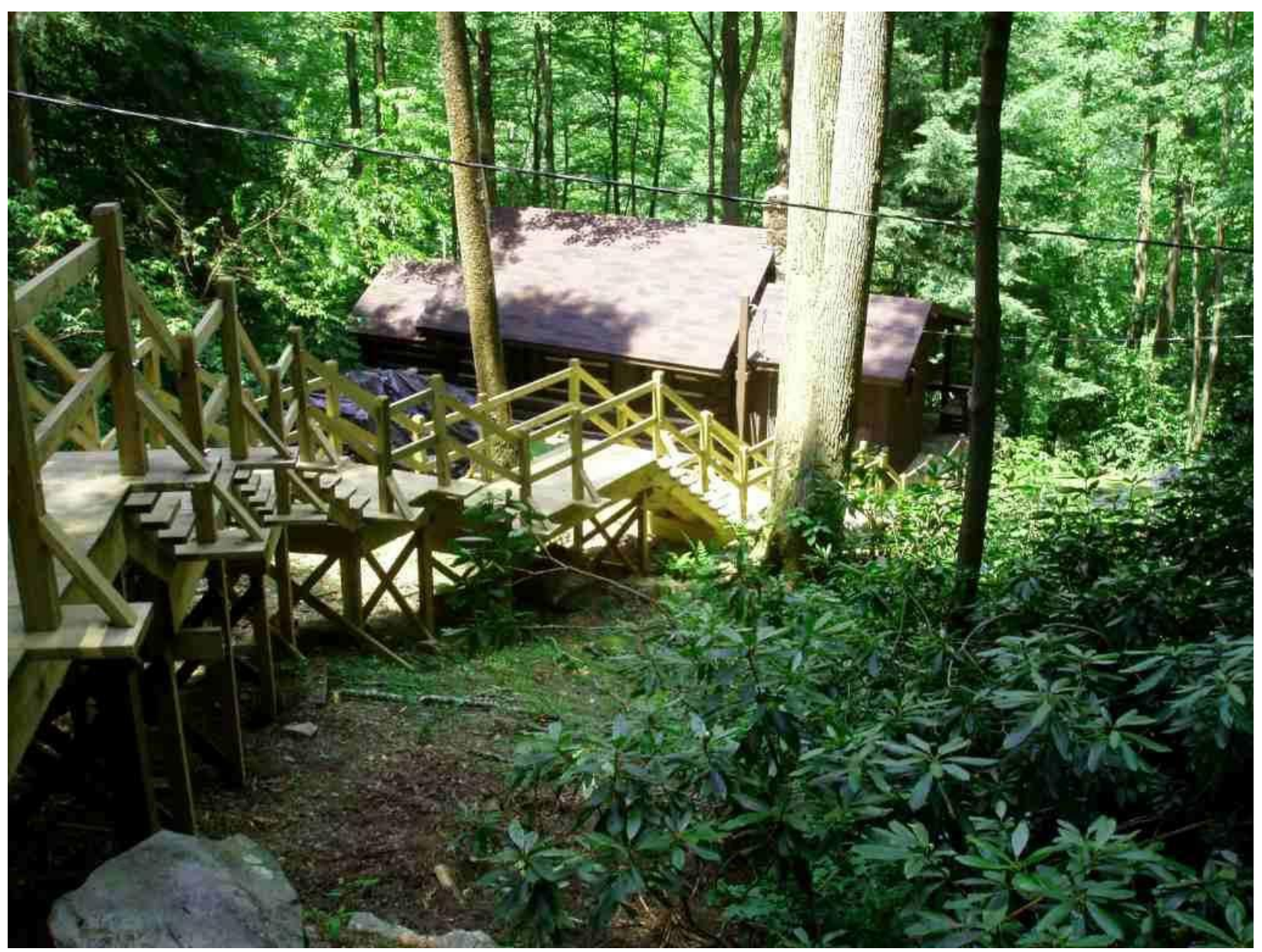

Figure 5.1. Partially obscured object (http://www.flickr.com/photos/jowo/15609025/) 
small hill in the landscape or a cabin may be obscured by a stand of trees (Figure 5.1). However, the fact that features are invisible and missing from a given view may be as relevant to an investigation of a landscape as the area that is visible (Higuchi 1988).

While visibility is a dominant component of traditional GIS landscape assessment, if not the sole determinant, the role of distance is often overlooked. When viewing a landscape, the acuity of the eye is critical, for distant objects are more difficult to discern and less detail is seen than objects that are nearby. In Higuchi viewsheds, distance is used to capture changes in the appearance of features in the landscape based on the Euclidian distance between the observer and the object observed (Higuchi 1988). The index is based on a categorization of short-distance, middle-distance, and long-distance reflecting an object's relationship and distance to the observer. These categories are defined specifically by the amount of detail that can be seen in the features within the landscape (Figure 5.2). Short-distance views represent areas closest to the observer in which distinct details of an object are visible such as the trunk, limbs, and leaves of a tree. Short-distance views thus provide the most detailed visual observation and contributes to the experience of a landscape since the observer combines observable detail with the additional stimuli of auditory and olfactory responses. A middle-distance view begins where the observer is no longer able to distinguish sections of the individual unit or its component parts, but instead sees the general outline of the whole object. Continuing to use trees as an example, the middledistance begins where it is no longer possible to see the details of individual trees, but individual trees or a stand of trees can still be discerned. Higuchi suggests that the transition from shortdistance to middle-distance occurs at approximately sixty (60) times the average height of an object. In a sprawling forest, for instance, an average tree height might be five meters suggesting that the break from short to middle-distance would occur at a distance of approximately 300 


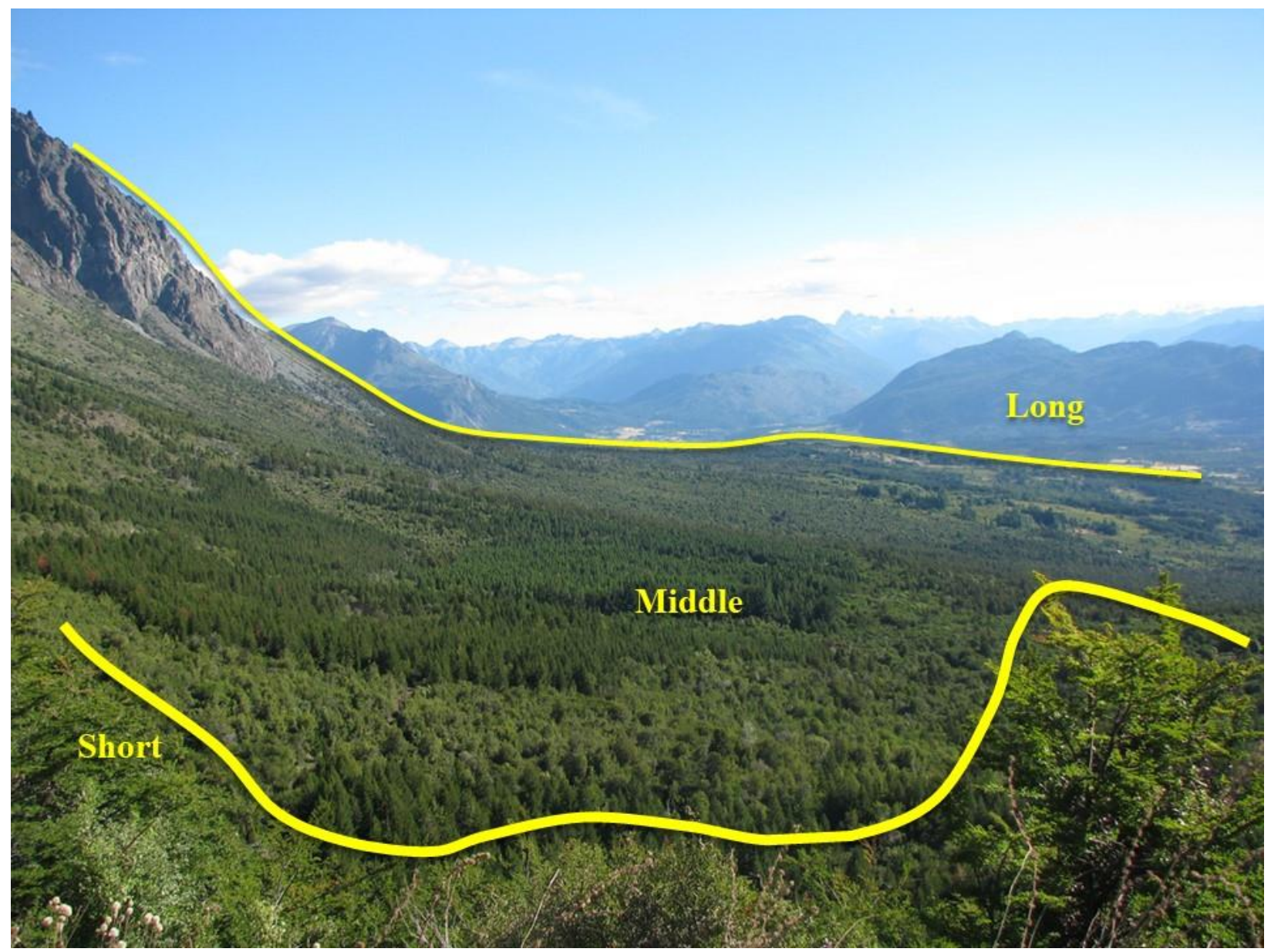

Figure 5.2. Representation of Long, Middle, and Short Distance (http://www.flickr.com/photos/23920588@N08/3264140465/)

meters from the observer. Higuchi suggests that the middle-distance represents an area that has the strongest role in defining our visual sense of depth in a landscape since it is primarily influenced by texture, light, and shadow, and is generally too far away for other human senses to come into play.

Long-distance views are defined by the human inability to determine even the general outline of specific objects such that features are seen only as part of the whole topographical feature in which they exist, such as a forest. Here, other aspects impact the viewable scene such as atmospheric effects, including haze, fog, texture and color. These effects tend to dominate the 
short and middle-distance views yet are little more than light or dark blurs in long distance views. Due to the impact of these various external considerations including the height of objects and atmospheric conditions, Higuchi suggests that the break between middle and long-distance views could be measured as approximately 1,100 times the height of the object. Returning to our 5 meter tall tree, the break between middle and long-distance will thus occur at approximately 5.5 kilometers from the observer. The role of short, middle, and long- distance perspectives in any consideration of the visual perception of landscape helps to determine not only what falls within the visible area, but the extent to which objects or features in the landscape can be seen, recognized, or experienced. This distance factor in viewshed assessment contributes markedly to our experience of landscape and yet is nullified in most GIS viewshed applications.

Many of Higuchi's other indices are based on the impact of a person's viewing angle when looking at a landscape. Higuchi's angle of incidence index measures the impact of planes and the various angles of the surfaces that make up the landscape being viewed. Based on the work of Gibson (1950), Higuchi suggests that viewed surfaces are made up of flat longitudinal planes and steep frontal planes. Other considerations such of angle of depression and angle of elevation come into play when determining the angle of incidence. If, for instance, an observer's focal point is assumed to be directly ahead, then a surface with a slope of less than $15^{\mathrm{O}}$ will extend into the distance adding depth to the landscape, while slopes greater than $30^{\circ}$ will appear vertical and may act as a boundary element within a landscape view.

While example values for the angles of incidence are based on the assumption that the observer is looking directly ahead, the angle of incidence can be modeled for other observation angles by including the angles of depression and elevation and acknowledging the physiological characteristics of the human eye and eyesight. Imagine a line radiating out from an observer at 


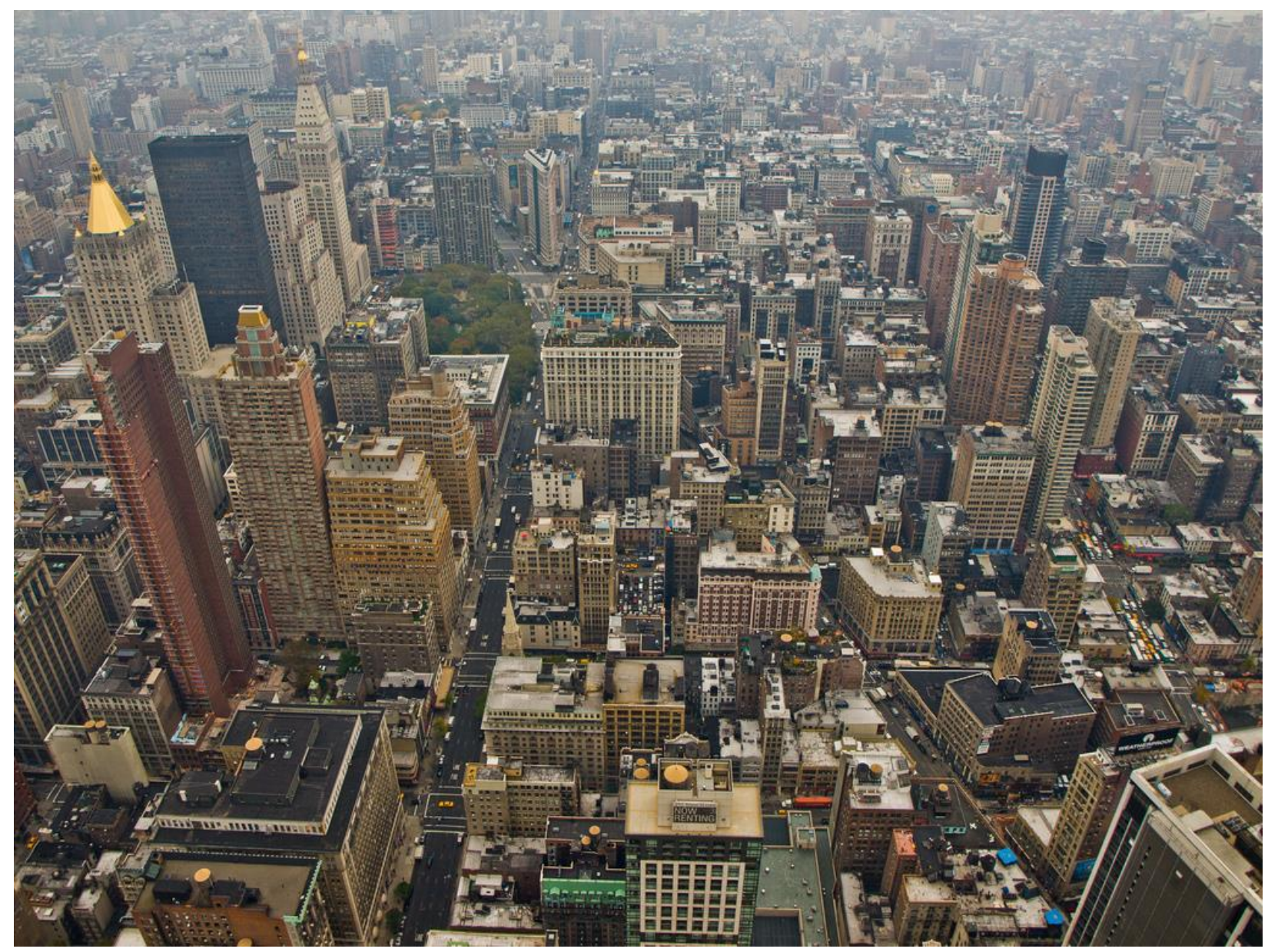

Figure 5.3. Angle of Depression (http://www.flickr.com/photos/wwarby/2229939903/)

eyelevel and label that $0^{\circ}$. Anything below that line is an angle of depression (Figure 5.3) and anything above is an angle of elevation (Figure 5.4). Higuchi (1988) points to research that indicates that the comfortable range of vision for the human eye lies between a $30-60^{\circ}$ angle below eyelevel and $30^{\circ}$ above (Figure 5.5).

The angle of depression is described by Higuchi as 'free and open' and has a significant role influencing whether we view an object as near or far. The view from a ridgeline invariably provides an open vista. Areas that are below the observer appear to be closer due to the steeper angle than those areas that are at a lesser angle and at a greater distance. The angle of elevation is 


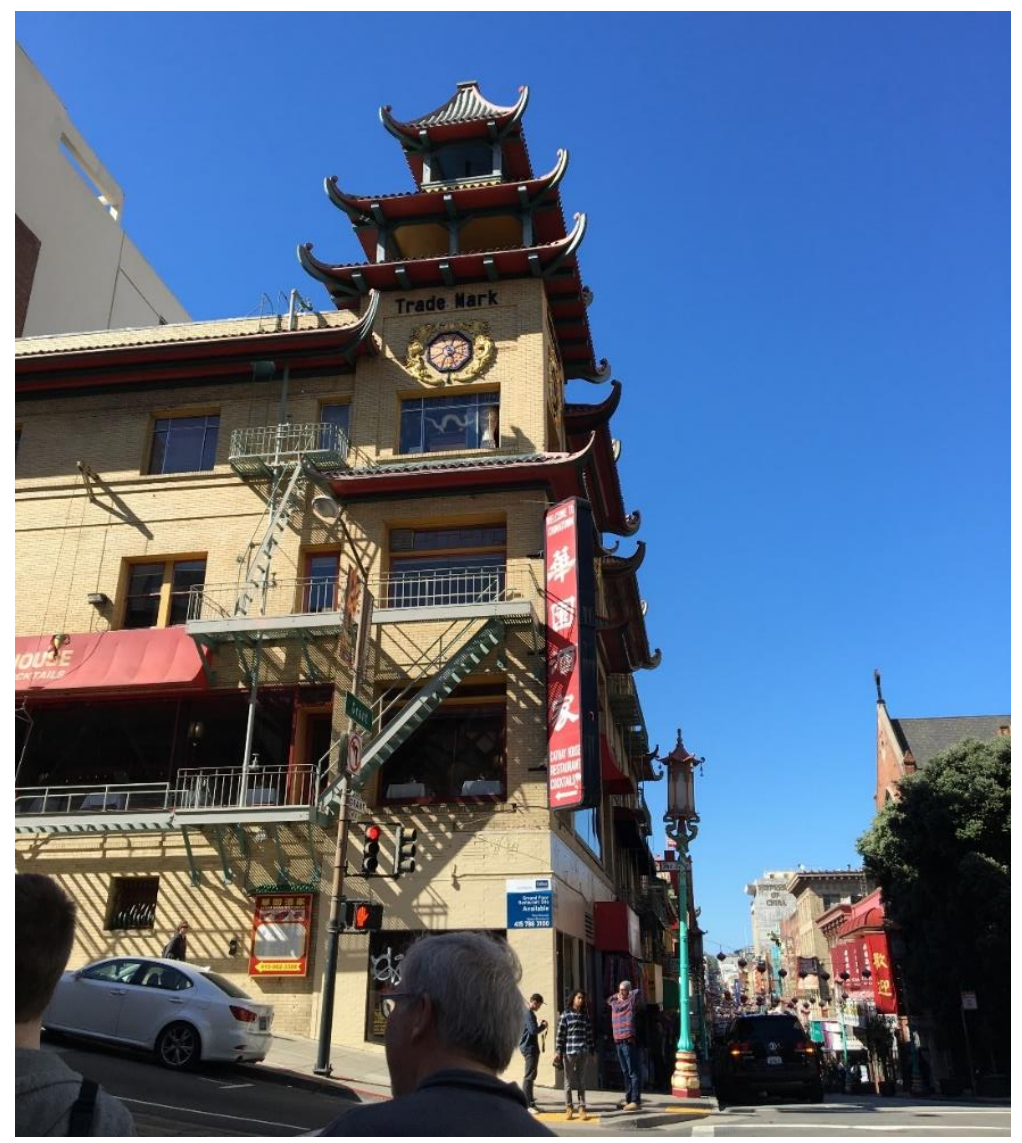

Figure 5.4. Angle of Elevation limiting since, if the observer is forced to look upward, the view is likely to be bounded in some way by the feature(s) being observed. In an urban area a vista could be limited due to structures that may force the eye upward, thereby physically bounding the space. When an observer looks above or below the horizontal $0^{\circ}$ eyelevel the angle of incidence will change in relation to the angle of view. As an example, if an observer is

looking down on a relatively gentle slope, the angle of incidence will be a combination of the angle of depression and the slope of the surface. Each of these indices provides a significant elaboration and sophistication over simply a line of sight approach and embeds the human into generating landscape viewsheds, and, as argued here, the experience of landscape.

Higuchi's depth of invisibility is a measure of the area of landscape that is hidden by natural or cultural features. This index provides a method for considering what is not seen in a landscape. Higuchi, as a landscape architect, suggests that the depth of invisibility is a way of designing visually unwanted objects in areas of a landscape that are hidden from view or alternatively that can be taken advantage of for example to deliberately hide unsightly objects such as transmission lines or for creating greater privacy. For the purpose of modeling the visual 


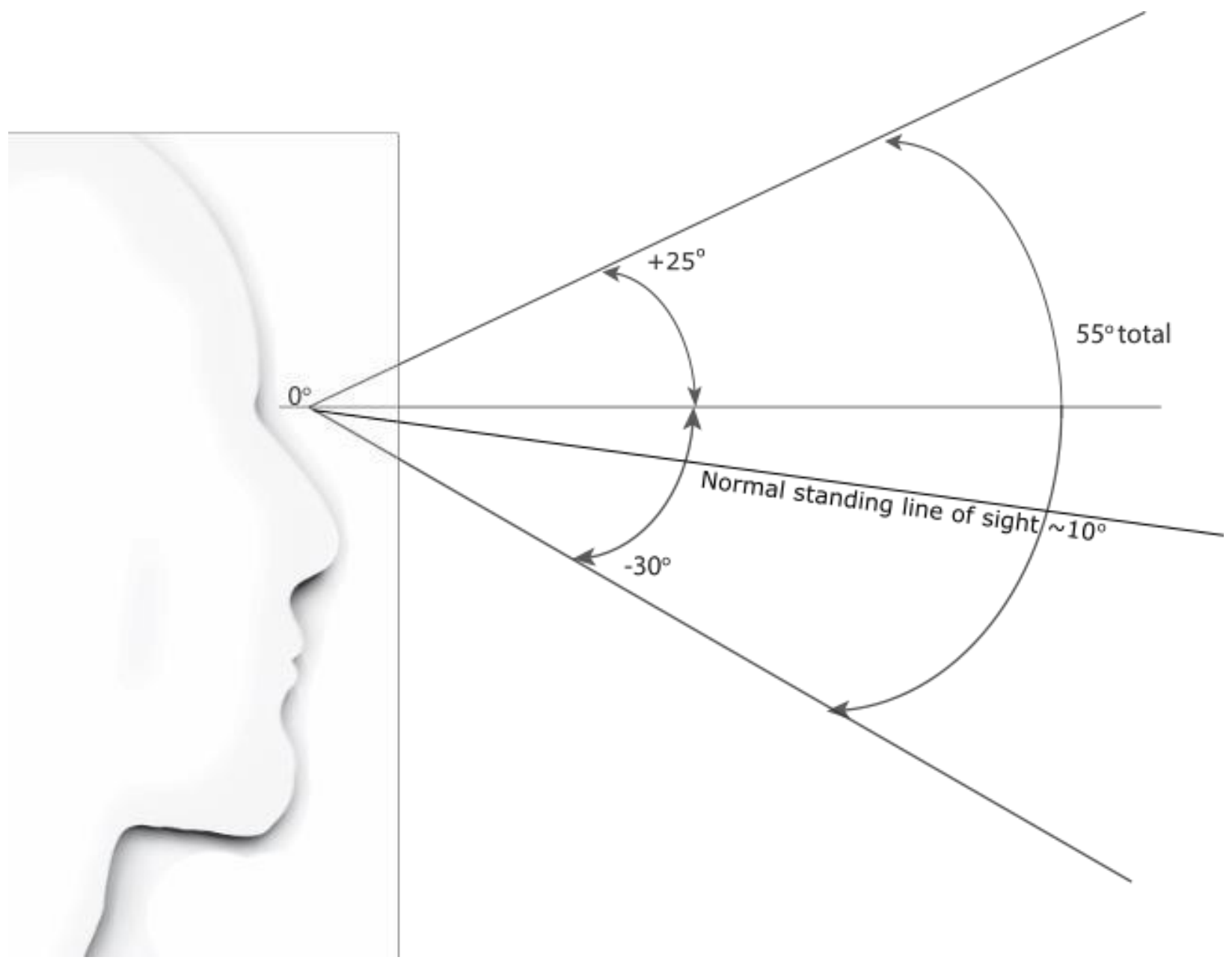

Figure 5.5. Visual range based on Higuchi

perception of landscape, however, the depth of invisibility could be used to define areas that may have been deliberately used by societies for particular cultural purposes (Higuchi 1988).

The measurement of depth is based on a set of factors including texture and the longitudinal and frontal planes discussed above. Depth reflects the sense of landscape 'volume' (as Higuchi describes it) that an observer perceives regarding the visible landscape that distance, as a Euclidian measurement, alone cannot convey. Texture also provides a sense of volume of the visible landscape by recognizing that differences in pattern, color, light, and shadow play into how we perceive landscape depth. For instance, leaves and branches with their hard edges 
represent a very different texture from that of a distant forest that is a conglomeration of objects and which appear 'softer' in appearance at a distance.

Atmosphere provides an additional visual cue to landscape depth and to the spatial depth index. Under most atmospheric conditions, objects that are further away tend to be obscured to a greater extent than those that are nearby. The descriptive naming of the Blue Ridge Mountains is a good example of this as the ridges that are closest to the observer are clearly tree covered, while those ridges that are farther away are obscured by haze and appear as blue outlines in the distance even on clear days (Figure 5.6).

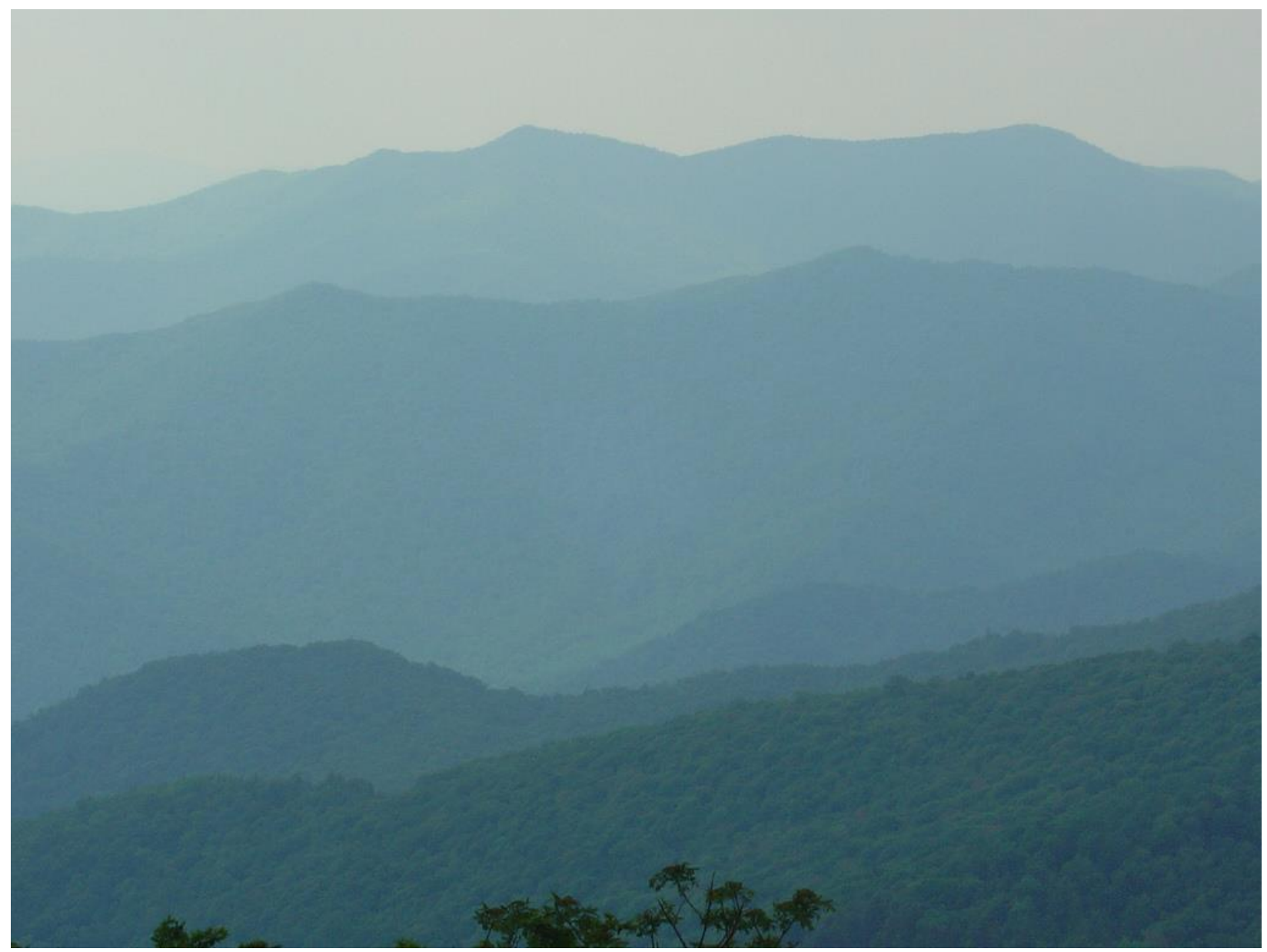

Figure 5.6. Atmospheric effects (http://www.flickr.com/photos/23502011@N08/2733100697) 
Finally, light, the last of Higuchi's individual indices, performs multiple roles in influencing our visual perception of landscape. Variations between light and shadow impact not only how well we can see parts of a landscape, but also our sense of depth. Atmospheric effects such as clouds often change the lighting conditions of a landscape creating radical alterations in the way an area is perceived even through the course of a single day. Similarly, our perception of landscape is influenced by diurnal changes and the angle of sunlight in the early morning, midday, sunset, or even by moonlight or under artificial light such as streetlights. Areas in shadow provide visual cues that are almost as strong as the features that cast the shadows. The shadowy alleys or streets between multistory buildings in urban areas carry with them certain emotional and atavistic responses that are partially tied to the lack of light and partially tied to the limited view caused by the angle of elevation (Turner et al. 2001).

While Higuchi's indices provide additional insight beyond a traditional viewshed into the physiological response to landscape, they also provide the basis of three composite analyses that combine the individual indices to consider visibility, depth, and occlusiveness. The Visibility of Landscape composite brings together the light and distance indices with the angles of incidence, elevation, and depression. The Spatial Depth of Landscape composite utilizes the textural, atmospheric, distance, and angle of incidence indices. Finally, the Spatial Occlusiveness of Landscape composite is created from a combination of distance, angle of incidence, and angle of elevation. Each of the composites builds on what can be understood through each of the individual indices and extends their potential by combining them.

Higuchi developed the eight indices and associated composites discussed above for use by landscape architects to enable them to assess how people view a landscape and how the various landscape elements can be used to measure or assess the visual structure of landscape: 
"The indexes proposed are intended to aid the designer in determining the function of these features, particularly those topographical elements that form the background or foundation of the landscape, and to examine their visual and spatial structure with relation to a development plan.” (Higuchi 1988: 5)

The applicability of Higuchi's indices for modeling the visual structure of landscape has been limited and only partially used by others outside of landscape architecture (Wheatley and Gillings 2000, LaKose 2004, Rouse et al. 2004b). To date the majority of attempts to implement Higuchi viewsheds have focused on two or three indices due to the issues that have arisen from data generation and from calculating measurements for all of the indices in a GIS software package. Wheatley and Gillings (2000) developed a simplified form of a Higuchi viewshed using GIS to create a distance-enhanced viewshed. This, however, ignored the subtleties that make the Higuchi viewshed such a powerful tool for landscape quality assessment. LaKose (2004), Harris et al. (2000) and Tschan et al., (2000) have all shown how a simple elevation model can be enhanced to support an understanding of the visible landscape: LaKose through the implementation of Higuchi viewsheds within a GIS, and Harris et al. by creating geovisualization reconstructions to allow users to virtually insert themselves into a past landscape. Tschan (2000), in looking for new approaches to landscape interpretation, found that perception and traditional viewsheds are not necessarily inclusive and suggested that new approaches to bolster traditional viewsheds may offer more meaningful information.

While Higuchi has provided a landscape assessment model, additional or differing metrics of landscapes could extend the model even further beyond its original specific landscape 
architecture purpose. The goal in this study is not to create a taxonomy of landscape types, but rather to develop a framework for capturing a sense of experience and place as part of a cultural interpretation of landscape and as promulgated by phenomenological archaeologists and landscape geographers such as Tuan (1974), Appleton (1975) and Tilley (1994) and to situate this within a geocentric GIS environment. Creating this complementary experiential and human centered viewshed component within a structured GIS approach to landscape that also enables a shared and replicable assessment of landscape that is so often missing from solely phenomenological interpretations is a significant step forward. 


\section{Chapter 6 - Situating the case study: the prehistoric landscape archaeology of Marietta, $\mathbf{O H}$}

Located at the convergence of the Muskingum and Ohio Rivers, Marietta, Ohio has been inhabited continuously for over three millennia. Though the cultures that occupied the area have changed significantly during that time, from early hunter-gatherers to modern city dwellers, there remain relict features in the landscape that are vestiges of the landscape's deep past. Notable remnants of the prehistoric landscape consist of extant earthen mounds. Weeson (1998) and others have described these mound groups as sacred landscapes, a material culture expression of social, political, and cosmological ideologies of the people who inhabited the land and which represent "the unique cultural milieu within which [the mounds are] created and used" (Weeson 1998: 95). The mounds offer a unique opportunity to study a local prehistoric culture that had such an impact in creating and shaping a cultural landscape of national significance.

The physical confines of modern Marietta provide an encapsulation of the landscape and mounds that form the core of the project area (Figure 6.1). The terrain sits within the Allegheny Plateau with local elevations ranging from 580 to 1,015 feet above sea level. The Muskingum River enters the project area from the north and joins the Ohio River along the southern boundary of the project area (Figure 6.2). The Ohio River enters the project area upstream to the southeast, bowing slightly northward, and exits the project area downstream to the southwest. There are several streams that flow into both the Muskingum and Ohio Rivers in, and around, the project area. 


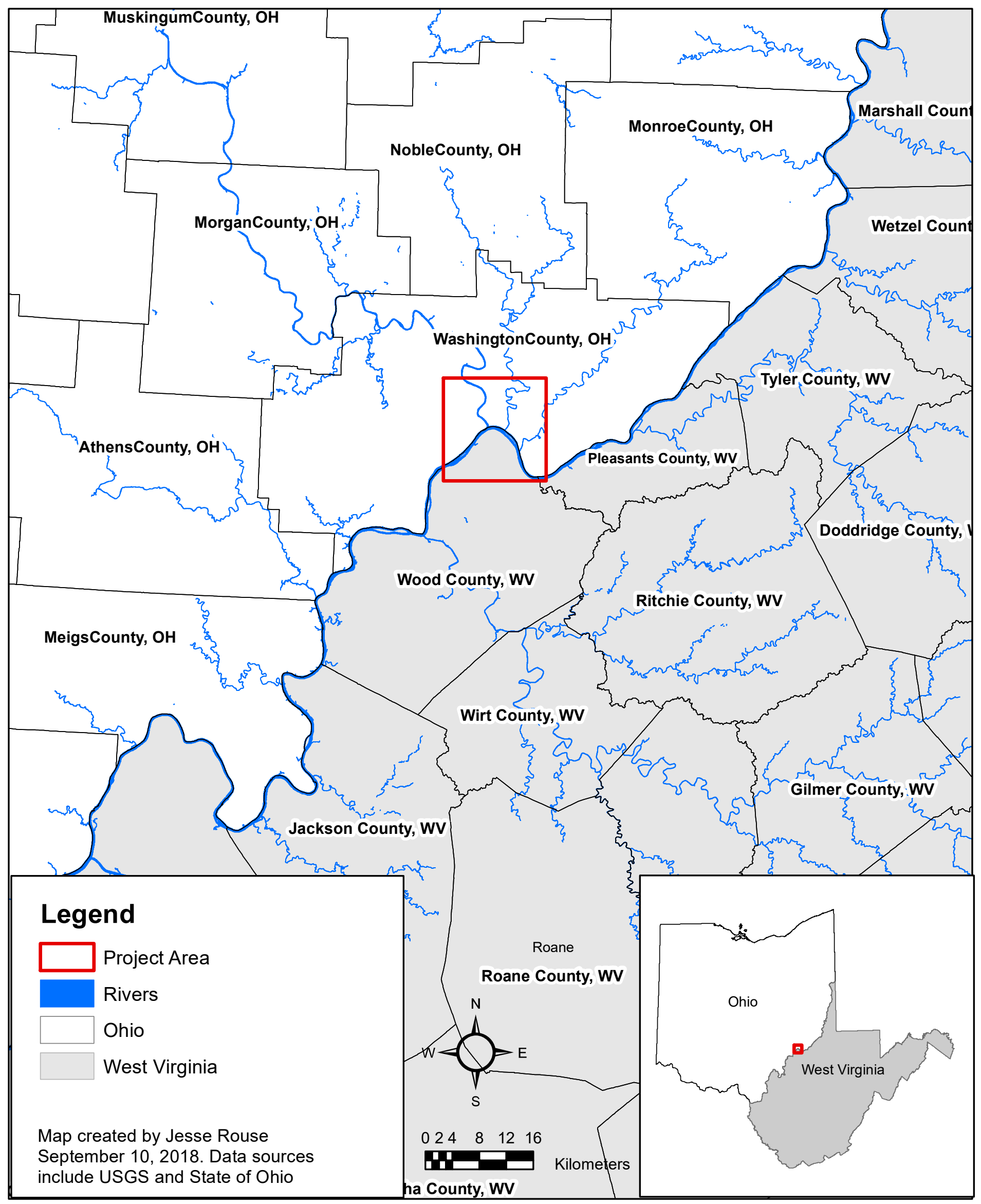

Figure 6.1. Location of the project area. 


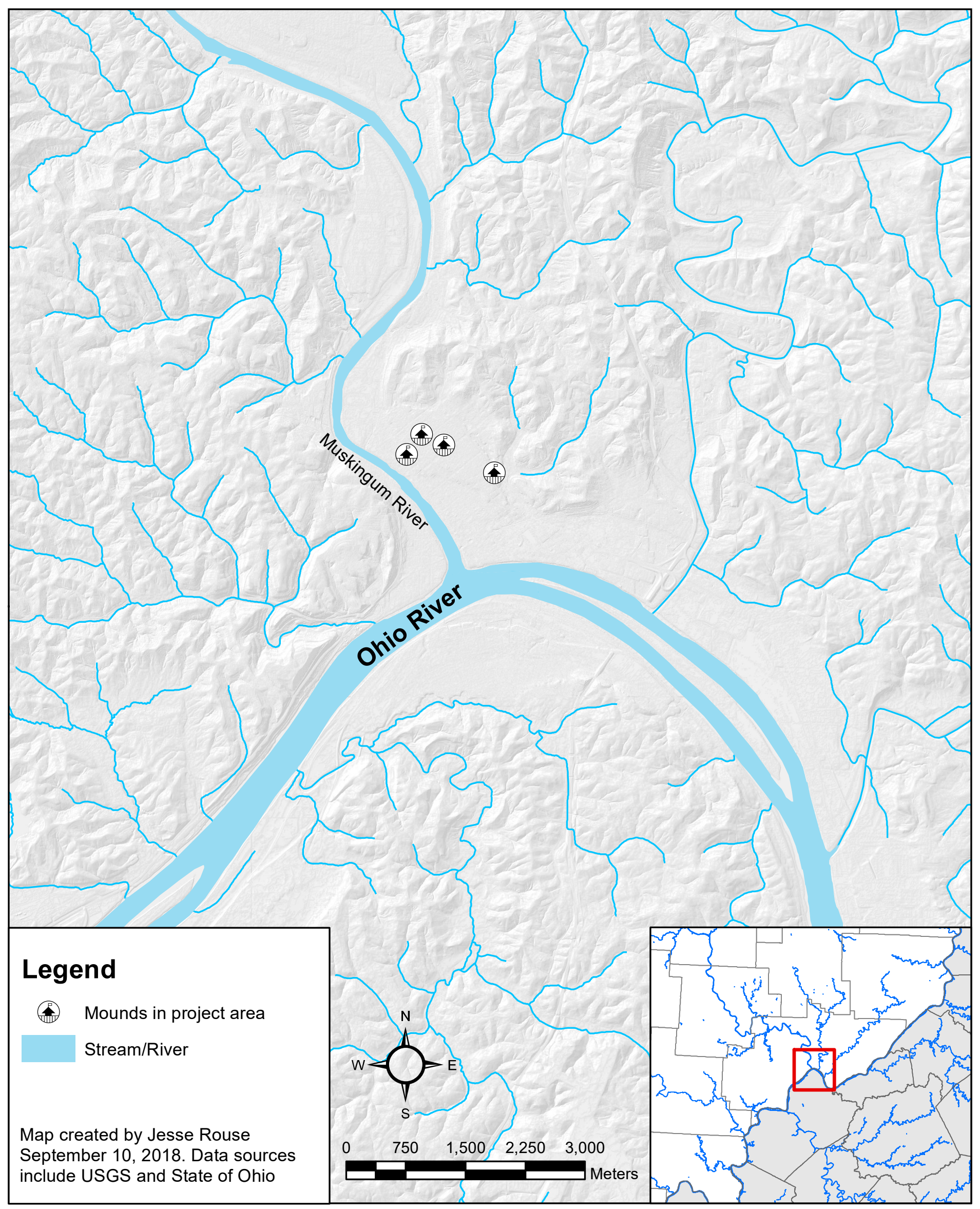

Figure 6.2. Overview of project area 


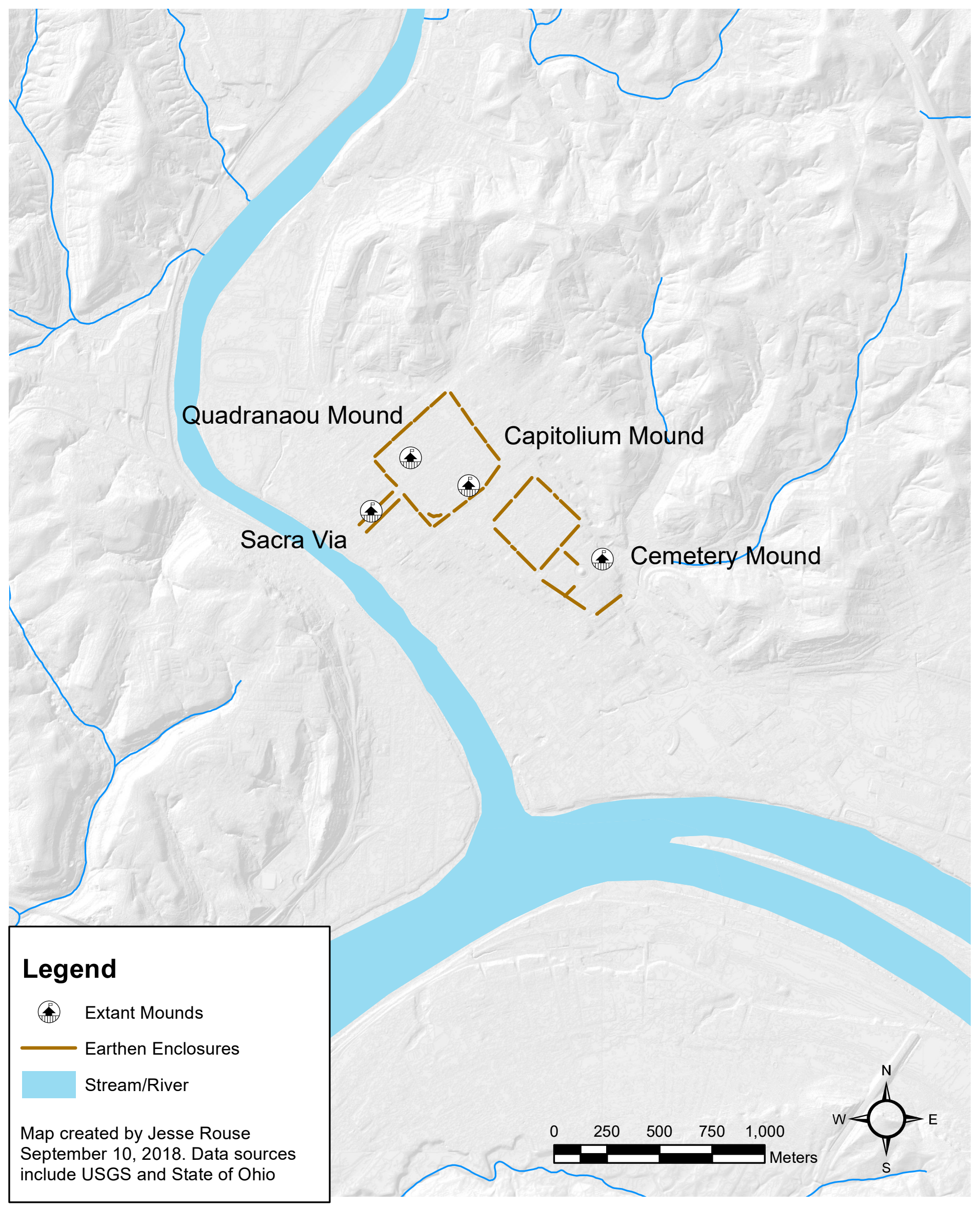

Figure 6.3. Extant mounds and recorded earthworks 
To the northwest of the Muskingum and Ohio River confluence, across the Muskingum from modern downtown Marietta, the area is made up of a small floodplain with terraces stepping up into the surrounding hills. The prehistoric focus of interest for this study lies on the broad, terraced floodplain to the northeast of the Muskingum Rivers' confluence with the Ohio River. In this area now sits Marietta's historic downtown, residential areas, and Marietta College. Three prehistoric earthworks remain extant, the conical Early Woodland mound known locally as Cemetery Mound, or Conus, and two Middle Woodland platform mounds known as Quadranaou and Capitolium (Figure 6.3). Records, drawings, and survey diagrams suggest that these extant features were a portion of a larger set of earthworks that included a series of walls that surrounded the mounds and a walled processional known as Sacra Via that extended from the river toward the Capitolium mound (Squire and Davis 1998).

The project area was likely forested for much of its past, though once agriculture was practiced more broadly in the Woodland period the area was likely cleared (Sauer 1944). Historic descriptions (Silverberg 1986) indicate that the area was forested when Europeans first ventured into the region. Trees removed from the earthworks in the $1700 \mathrm{~s}$ were estimated to be as much as 400 years old

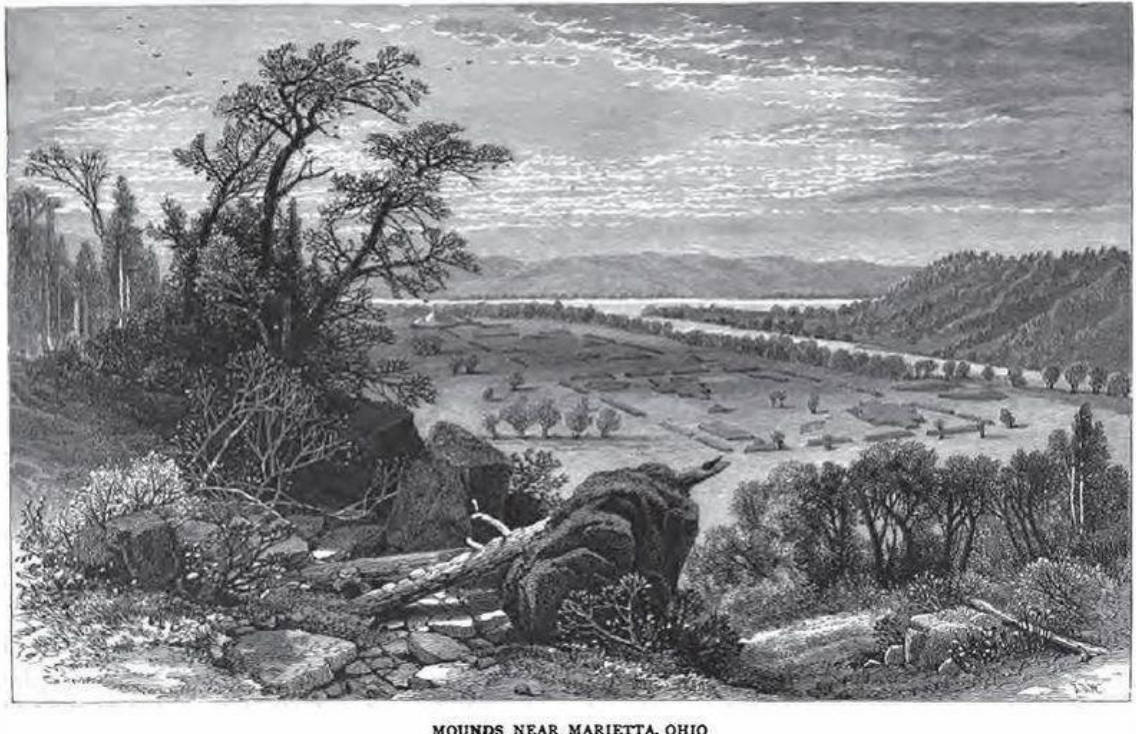

Figure 6.4. Marietta Mounds (Bryant and Gay 1888) 
based on a counting of the tree rings. Yet, drawings made by early European explorers show that the Marietta landscape was cleared early in the historic era and mostly treeless so that the earthworks stood out in stark relief in the landscape (Figure $6.4 \& 6.5$ ).

\section{Cultural history}

The area at the confluence of the

Muskingum and Ohio Rivers possessed

multiple natural features that attracted

early people to it. The area was likely an

intersection along one of the first major

transportation corridors in eastern North

America, the Ohio River. There are rich

soil deposits along the river valley left

by occasional flooding which have for

millennia been utilized for agricultural

purposes. As shown through the

construction of the mounds in the area,

the place likely held spiritual

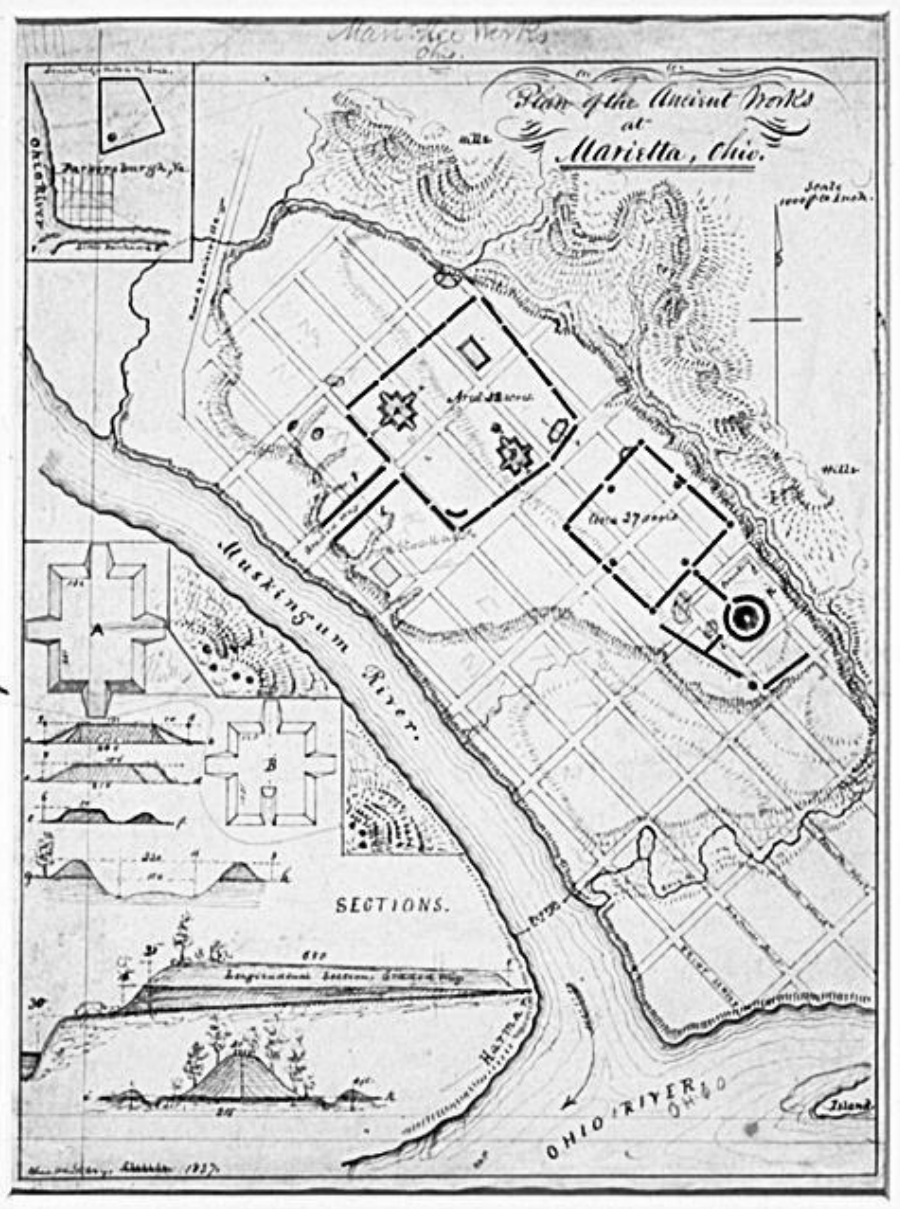

Figure 6.5. "Plan of the Ancient Works at Marietta, Ohio," by Charles Whittlesey, 1837 (Squire and Davis 1988)

significance for the inhabitants and was

used for sacred purposes that are linked to these mound features (Goad 1979). These mounds, along with other physical and cultural factors, attracted native peoples into this region and, in turn, connected them to larger cultural groups. 
The Marietta area is located in what is archaeologically referred to as the Eastern Woodlands which is the region of North America east of the Mississippi River that was heavily forested for most of the Holocene. It is firmly accepted that native peoples have been in the region for at least the last 10,000 years with evidence from various sites, including regional locations in Pennsylvania and Virginia, pointing to movement into the region even earlier (Bradley and Stanford 2004). The livelihood of these earliest peoples focused on nomadic huntergatherer activities following herds of wildlife throughout the region. During this early PaleoIndian period, which extends from the introduction of people into the region to around 10,000 before present (BP), the population relied on the megafauna in the area, such as mammoths and other large animals, for their survival, supplemented by the gathering of flora. The climate 10,000 years ago was that of an ending ice age with the terminus of the Wisconsinian and a landscape that was heavily impacted by glacial melt. There is evidence of Paleo-Indian activity near the project area represented by isolated finds of Clovis spear points that provide a relative, but not an absolute, dating of human activity (Murphy 1989).

Divided into early, middle, and late, the Archaic period extends from the end of the PaleoIndian period at approximately $10,000 \mathrm{BP}$ to $3,000 \mathrm{BP}$. There was a significant alteration in the climate over this 7,000 year period from the warming that followed the end of the ice age, through the change of the altithermal and the eventual moderation to that of the modern climate approximately 5,000 years ago. During the Early Archaic period, hunter-gatherer subsistence shifted from the then extinct megafauna to large game such as deer and elk, with increased reliance on foraging and gathering in the Middle Archaic. Toward the Late Archaic the trend was toward horticulture in response to a cooler, moister climate. With the shift toward subsistence horticulture, native groups settled the area and, less driven by the pursuit of game, they focused 
on encouraging local flora populations (Gremillion 2004). The trend toward sedentary living and increased reliance on gathering and horticulture, as opposed to the earlier focus on hunting, allowed communities to become established and for cultural traits to evolve within those communities.

In the Late Archaic period evidence emerges of two cultural trends that eventually impacted local populations in the Marietta area. The first trend was that of the rise of ceremony associated with burials and the cultural attachment to community and place that these ceremonial processes revealed. The inclusion of funerary goods in burials in this period suggests a cosmological perspective and a desire to honor and recognize those who had died (Sassaman 2010). The second trend can be discerned outside the study area along the Green River in Tennessee and Kentucky where conical shell heaps were created from food refuse, and which begin to take on shapes and heights that go beyond simple functionality (Claassen 1998). In addition to the creation of shell mounds, the location of middens along the river make it clear that a relationship existed between native groups in the region and the lower lying areas in this dissected landscape. Earlier Paleo-Indian groups and Early Archaic materials are often evidenced in areas that have prominent views of the river valleys; however, during the Middle and Late Archaic periods, people appear to have migrated into the river valleys in order to benefit from better soils, the river water, and the wildlife that were attracted to the rivers (Sassaman 2010). During the Early Woodland period, beginning around 3,000 years ago, subsistence in the region was transitional with evidence of continuing horticultural and hunting activities in conjunction with the establishment of agricultural practices and products such as gourd squash and sunflowers (Peregrine and Ember 2002). The practice of agriculture contributed to 
populations becoming sedentary and tied to particular areas. The shift to a sedentary lifestyle also leads to technological shifts such as the introduction of pottery.

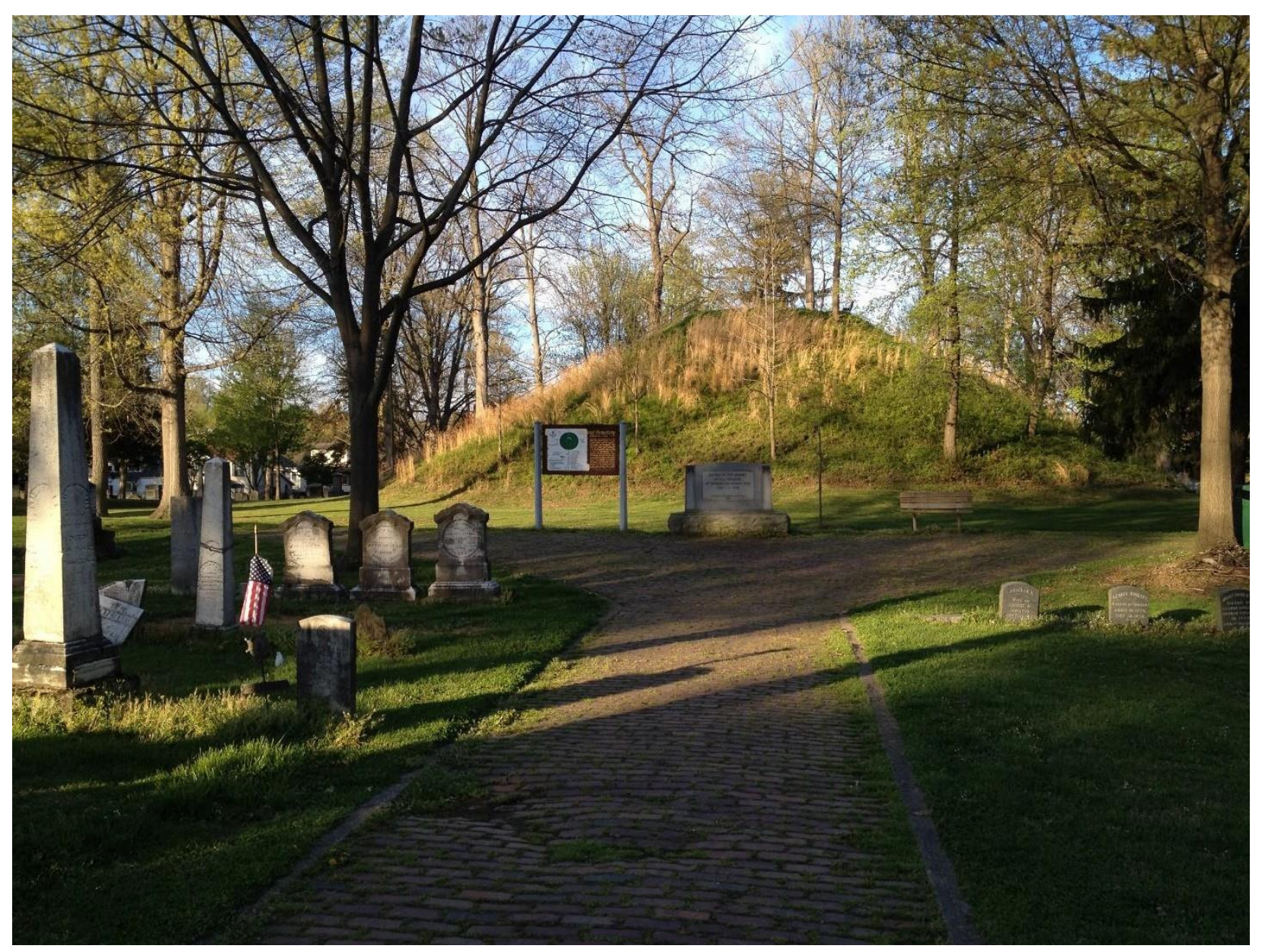

Figure 6.6. Cemetery Mound in Marietta

Today in Marietta, Cemetery Mound (Figure 6.6) remains a dominant symbol of the Early Woodland period and of the 'Adena' culture that existed in the region. The Adena culture is typified by conical mounds of various sizes built atop a burial that usually includes funerary goods. These mounds are accepted as a physical representation of a cosmological outlook that is described as sacred (Korp 1990). Cemetery Mound, based on existing archaeological and visual evidence, represents the kernel of the Marietta sacred cultural landscape (Summers 1903). 
The cosmology surrounding these mounds and the landscape in which they were set took on greater prominence in the Middle Woodland, beginning approximately 2,000 BP. The Middle Woodland period saw a change in the construction of the mounds from conical burial mounds to platform mounds that are often ascribed to ceremonial use with only the occasional burial. Another change in mound structure was the creation of mound groups that often resulted in Hopewell mounds being located near or adjacent to existing Adena mounds. As the Adena culture is representative of the Early Woodland mound builders in the region, so the 'Hopewell' culture is linked to the mounds of the Middle Woodland period. These Hopewell Mound groups also commonly have external features such as connected walls and moats that often encircle and enclose the mounds (e.g. Figure 6.3).

Marietta has examples of each of these types of mound construction; proximity to an Adena mound, platform mounds, and enclosures. The only notable exception to these mound types in the area is the absence of effigy mounds in, or near, the project area. Effigy mounds are abstract representations of natural objects, often animals, which are generally best seen and identified from the air. The majority of effigy mounds exist to the west of the project area through central and western Ohio, Indiana, Illinois, and Wisconsin. This pattern reflects a shift in core activity between the mound building cultures of the Early and Middle Woodland periods. While the Adena mounds are most abundant in the upper and middle Ohio River Valley and its tributaries, the Hopewell mounds trend through the lower Ohio River Valley and upstream along the Mississippi River Valley from its confluence with the Ohio River.

The shift from Early to Middle Woodland is also associated with a deeper reliance on agricultural food sources. There is strong evidence through the development of mounds and excavated sites that community groups in the area were sedentary and attached to the places they 
occupied. However, while there seems to be a closer link to the local area, an expansive trade network becomes apparent during the Middle Woodland as indicated by funerary goods in various Hopewell sites in Ohio that include conch shells from the gulf coast region, mica sourced from the mountains of North Carolina, and copper from the Great Lakes region to the north (Ehrhardt 2009). This 'Hopewellian Interaction Sphere' extended through much of North America east of the Mississippi River with objects associated with the Hopewell culture found in other regions. Technological shifts in the Middle Woodland period are represented by functional objects, such as projectile points and pottery, and an increased number of craft goods, often made of non- local materials such as the conch and copper listed above, as well as regional clays and stone. These materials, both exotic and local, were abundant and there is evidence of daily use rather than a sole dedication to sacred or burial purposes. For malleable materials like copper, there is evidence of an ever-increasing level of artisanship that can be seen in various finds at Hopewell sites over time (Ehrhardt 2009).

While the majority of the mounds constructed in the project area occurred during the Early and Middle Woodland periods, this does not imply that the cosmological connection of native people to the mounds was lessened during the Late Woodland $(1,600-1,000$ years ago) or Late Prehistoric (1,000 years ago to contact). On the contrary, the Mississippian mound builders of the middle and lower Mississippi River valley and the southeastern portion of North America continued the construction and use of mounds through later periods, and it is likely that the importance of the mound features through the Ohio River Valley and tributaries continued to have an impact on local peoples. Even today these mounds are imbued with meaning, often not that of their original creators, but nonetheless they have a distinctive role in defining the place of both historic and modern Marietta. 
Today the locations of the extant mounds are highlighted through historic markers

(Figure 6.7). Sacra Via and Quadranou are now located in an arboretum and a park in the middle of the modern town. Capitolium lies as part of the base and foundation of an early 20th century Carnegie library. Cemetery Mound is surrounded by the oldest European cemetery in Marietta where Revolutionary War soldiers and frontiersman are laid to rest. Each of these locations conveys new meaning to the prehistoric constructs as the archaeological remains have been adapted and adopted into the modern urban backdrop. This adoption of the features, and the new connections that have been created, is perhaps the only reason that these mounds have remained extant in the landscape. Drawings and surveys of the area show that additional earthworks, mounds, embankments, and ditches existed but these have been consumed and obliterated by the growth of Marietta. Even Sacra Via, which is now a three block long park, no longer exists as it once did since

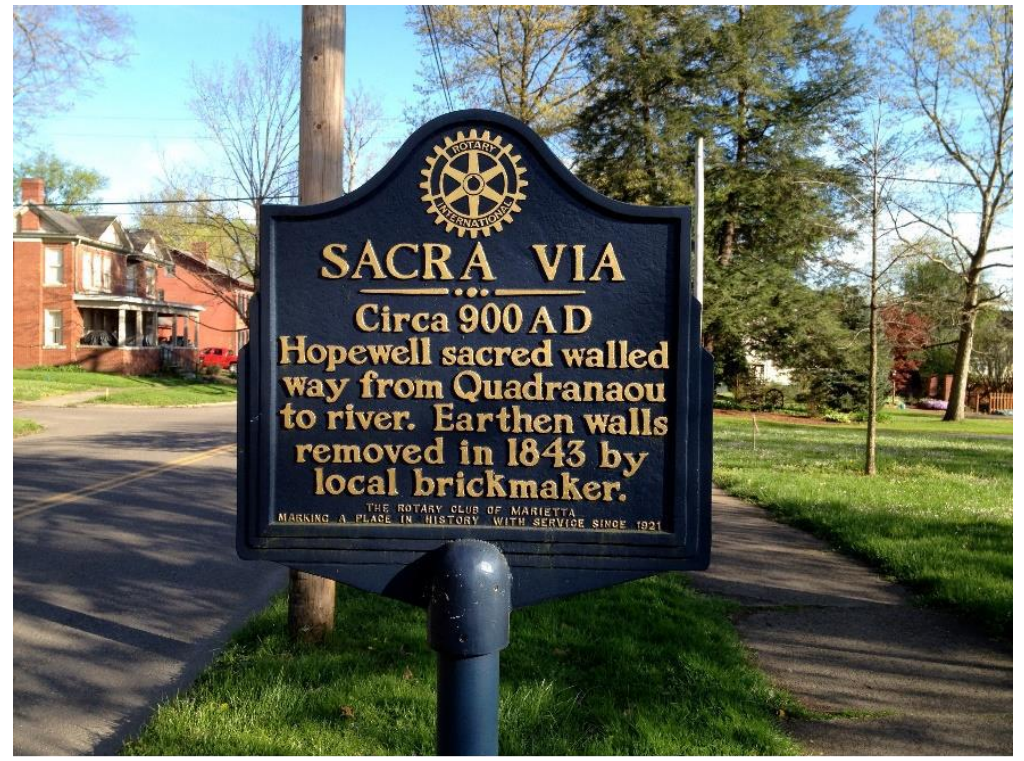

Figure 6.7. Historical Marker for Sacra Via the clay of the built walls of the ancient promenade became the source material for many of the later historic brick streets and homes in the area (Gerber 2008). Today, the prehistoric landscape is represented by a very few, but notable, examples of the original Native American constructions. These few representations, along with a myriad of historical markers scattered throughout the town, make up the modern experience of the relict landscape of Marietta. While 
providing a venue for tourists, students, and locals, it is clearly but a ghost of the landscape it was prior to European influence.

\section{The Rivers}

The contemporary Muskingum and Ohio Rivers are also different from their earlier forms due to a series of historic and modern locks and dams and the regular attention from the U.S. Army Corps of Engineers (USACE 2013). Today the water

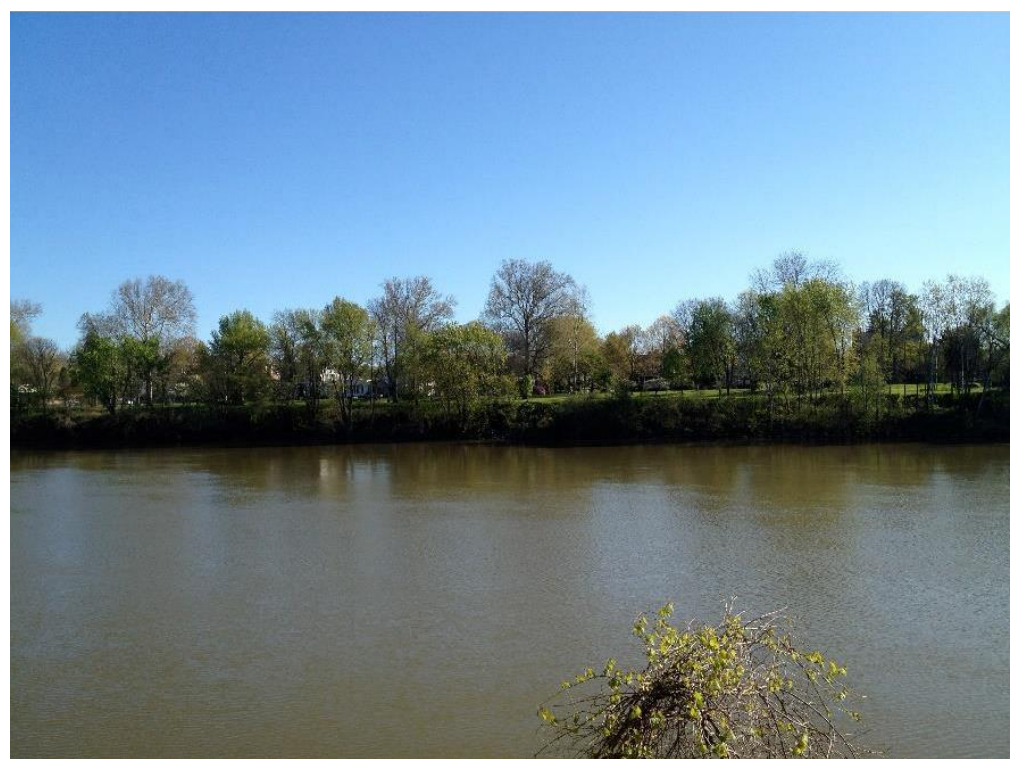

Figure 6.8. Looking across the Muskingum River toward Marietta levels remain relatively constant and the sloping riversides have in many places been altered over time to form an imposing wall of soil that reaches up high above the river anywhere from 10 to 20 feet at normal water levels

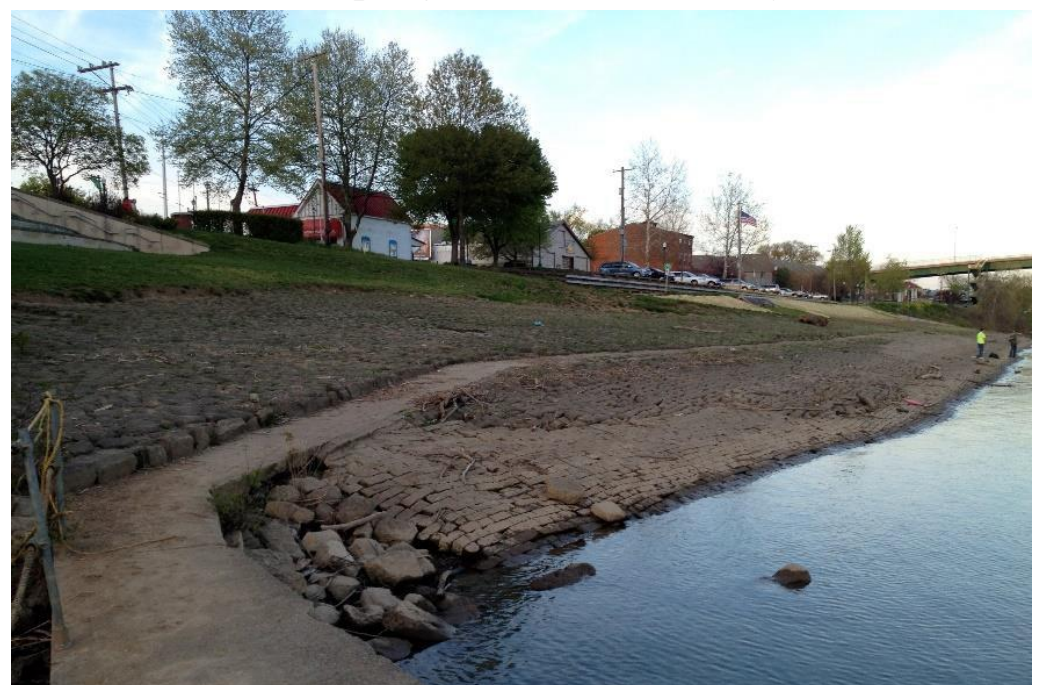

Figure 6.9. Ohio River front looking up toward downtown Marietta

(Figure 6.8). North of the project area the Muskingum River flows in a sinuous path cut into surrounding hills, limiting the visibility upriver on the Muskingum, as it approaches its confluence with the larger Ohio River in the southern part of the project area. The Ohio River generally has a gentler sloping bank reaching up to Marietta, and 
thanks to historic boat tie- offs (Figure 6.9). The riverside is lined with rock on the Marietta shore. On the opposite shore leading up to modern Williamstown, WV, the slope leads down to a sand beach on the river bank (Figure 6.10).

Today the Muskingum and Ohio Rivers are used for recreational and commercial boat traffic, especially the larger Ohio River. While bridges have connected the two sides of the Muskingum and Ohio Rivers for over a century, prior to that a ferry, barge, or boat was required

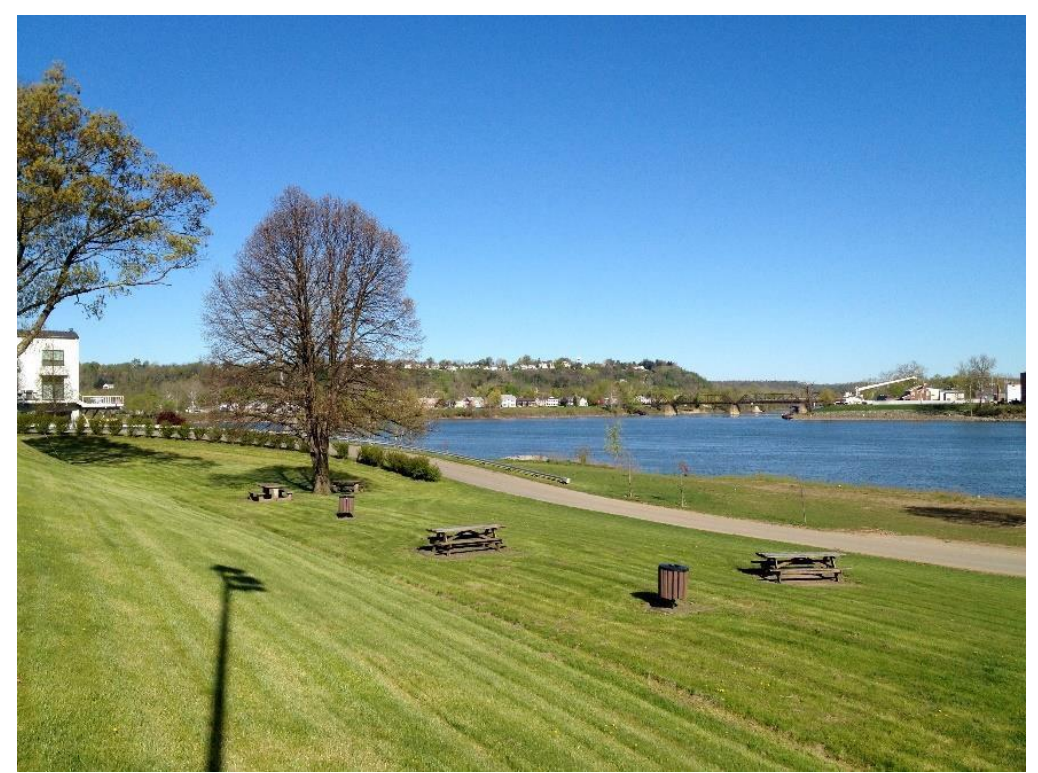

Figure 6.10. Williamstown, WV looking across the Ohio River toward the confluence with the Muskingum River to cross the river in most seasons.

However, in exceptionally dry periods, prior to the construction of the lock and dam system, the river could at times be easily forded due to low, inconsistent water levels. During the period when the mounds were in use, the rivers were likely an important part of the transportation system through the region. With no pack beasts or riding mounts, transportation in North America was largely limited to walking and boating. The project area was thus the equivalent of a modern interstate on-ramp as the smaller Muskingum River enters the Ohio River.

This native transportation network would suggest that a significant portion of visitors to the prehistoric project area may have arrived by water. With 2,000 years of natural change and the influence of historic activities along the river such as by the Army Corps it is difficult to tell exactly how the prehistoric population approached the area. Historic representations do suggest, 
however, that a landing existed on the bank of the Muskingum that led into the processional of Sacra Via (Squire and Davis 1998). 


\section{Chapter 7 - Experiencing the landscape of Marietta}

Tilley, one of the foremost scholars of the use of phenomenology in archaeology, describes a 'phenomenological walk' as a dualistic process whereby walking and writing become synonymous. During the walk, the landscape "unfolds in the form of a story or a narrative that needs to be written as one walks" (Tilley 2008:269). Stepping into a landscape, Tilley suggests, an observer would narrate a personal interaction with the landscape through the elements of intention and action. Intention and action can range from recording the material to noting the visual, from considering the sacred to the mundane. However, it is the sum of the parts, the context of the landscape that binds the story of the landscape as experienced.

Tilley, and other phenomenological archaeologists, suggest that the record created during a personal experience with landscape, along with the known information about an area, accentuates an observer's perception of the area, moving one from a natural, almost subconscious, awareness toward a deliberative, focused perspective of the seen landscape. As with the inherent issues associated with the participant:observer relationship in ethnography, the experience of landscape changes the truest interaction to be had by the observer through the actual alteration brought about by the experience itself. Tilley suggests that in the landscape, "the act of writing slows experience down and focuses attention." (Tilley 2008:269). This change is not in itself a detrimental point, but just as the participant:observer must be mindful of the biases that interaction with an individual or community can introduce, so must the cultural geographer be mindful of how their observations, at pace or at rest, detaches them from understanding the experience of landscape. Before seeking to create a phenomenologically empathetic approach to viewshed analysis using Higuchi indices connected to a GIS, an experiential walk is made of 
Marietta Ohio based on Tilley's approach. In particular and relevant to the Higuchi approach the nature of short, medium, and long vistas, the role of texture and color in the landscape, and the angle of viewpoint are examined in the field as to a prelude to the modeling and study.

\section{A personal walk through Marietta}

I begin my meandering experience of the extant archaeological features of Marietta on the bank of the Muskingum River over a kilometer upstream from the confluence with the Ohio River, and at the lowest point of the park representing Sacra Via. Sacra Via is documented as providing a processional between the Muskingum River and the upper terraces where the mounds are located. Though no longer extant, Sacra Via is historically illustrated as two embankments paralleling a path (Figure 7.1). Today, Sacra Via is no longer the impressive enclosed processional that it was but an open park and arboretum. The arboretum, which covers most of the park, provides the modern observer with an overview of local flora and a vista of the surrounding neighborhood and connecting roads and parks. However, it shows little evidence of the former ceremonial earthwork itself.

As I walk along the modern street I can see that the park runs on a slightly different orientation from that shown in the early mapped representations of Sacra Via. This is notable in that it suggests that in its original orientation there was a significant connection to the confluence

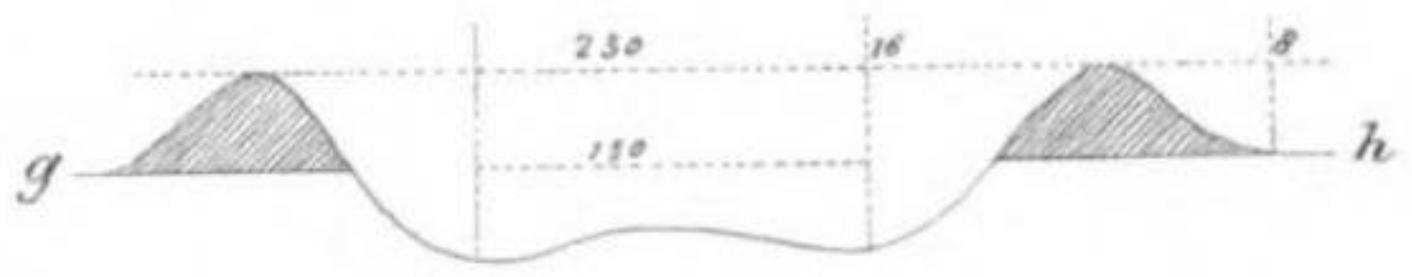

Figure 7.1. Cross-section of Sacra Via from Whittlesey's 1837 survey 
of the two rivers. The Ohio River, as a larger and more important waterbody, was likely the greater source of traffic to the mounds accessible along the lower Muskingum River.

Using the cross-section (Figure 7.1) provided by Whittlesey (Squire and Davis 1998) it is possible to consider what an experience from within the promenade may have been like. I can imagine that the walls would have been confining, limiting the view for someone coming from the river. Visitors to the site would have looked back to the river, but to either side visitors were presented with substantial walls of earth which would have directed their view uphill to the mounds and the hillside that was their backdrop along with the skyline. With so many Native American legends attributing importance to water, land, and sky, it is likely Sacra Via provided an allegorical representation of each of these themes.

As I proceed from the bank of the Muskingum River along the park that was Sacra Via, there remains even today a sense of expectation despite the area being surrounded by historic and modern buildings. With Whittlesey's historic survey map in mind (Figure 7.2) there is an expectation

of a grand entrance into the area of Figure 7.2. Whittlesey's 1837 historic survey map of Marietta

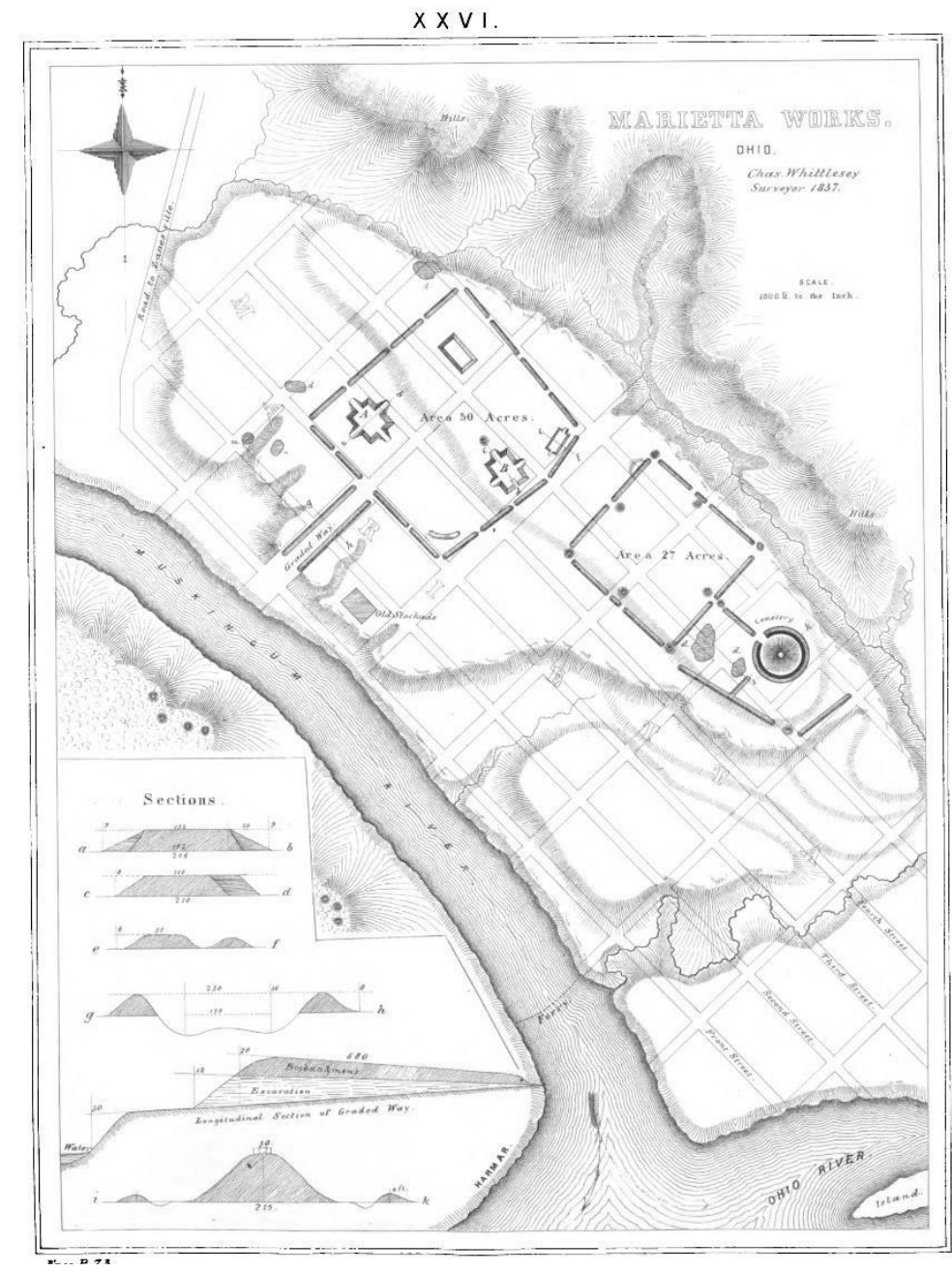


the mounds enclosed by a series of embankments. Some sections of the embankments surveyed by Whittlesey, such as a small section adjacent to Cemetery Mound, appear to remain (Figure 7.3), but the vast majority of the enclosing embankments are now gone, replaced by houses, lawns, roads, and other modern construction. Instead, as I look uphill, it is the lip of a river terrace that has a limiting effect on my view of the mounds.

It is not only Sacra Via that was impacted by the construction of the historic neighborhood that surrounds me, even the large platform mounds have been significantly impacted or destroyed by urban development. Only two mounds have survived relatively unscathed, Cemetery Mound and Quadranou. While Capitolium Mound is extant, I see that it is now the foundation of a later historic construction. Whittlesey's survey showed two additional large platform mounds and several smaller earthen structures that are no longer extant or are hidden beneath the dense historic housing that exists. Walking through the neighborhood I am hopeful that I might find

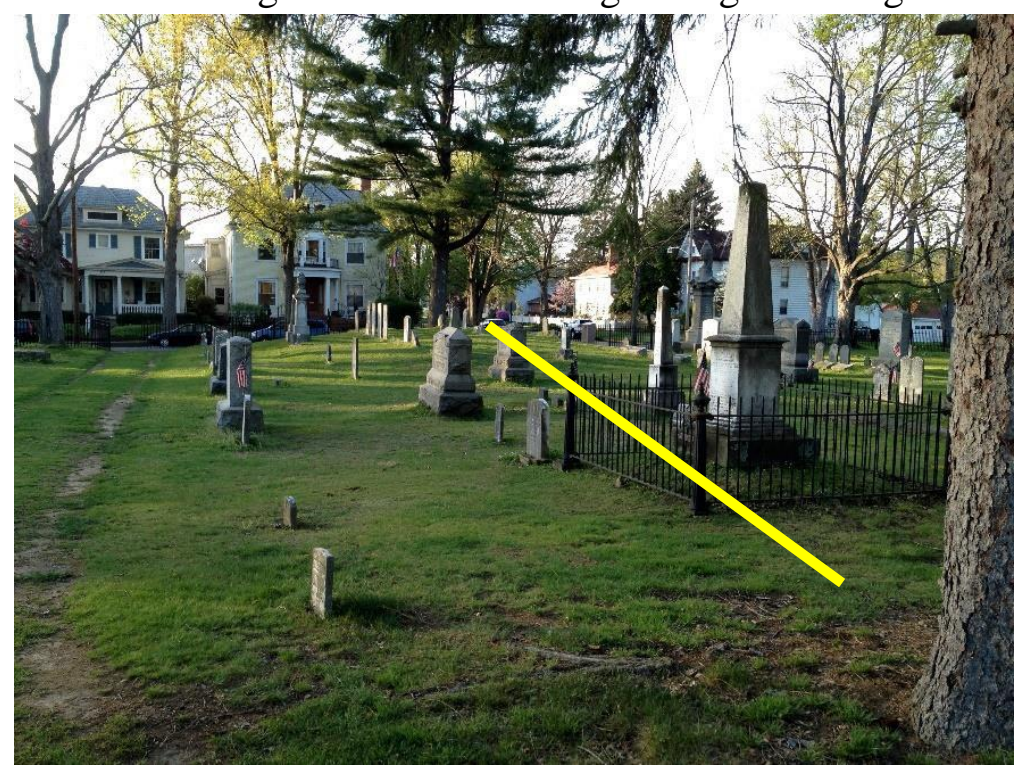
some small remnant, or at least a recognition, of the destroyed mounds. Alas, no markers or identifiable remains are in evidence amongst the lawns and streets.

Figure 7.3. Minor remains of one of the interior embankments 
At the end of the Sacra

Via processional, where the

earthen embankments would

have opened up into the

enclosure, stands what is known

as the Quadranou Mound. I

stand in the southwestern corner

of an open and grassy park

covering a full city block with

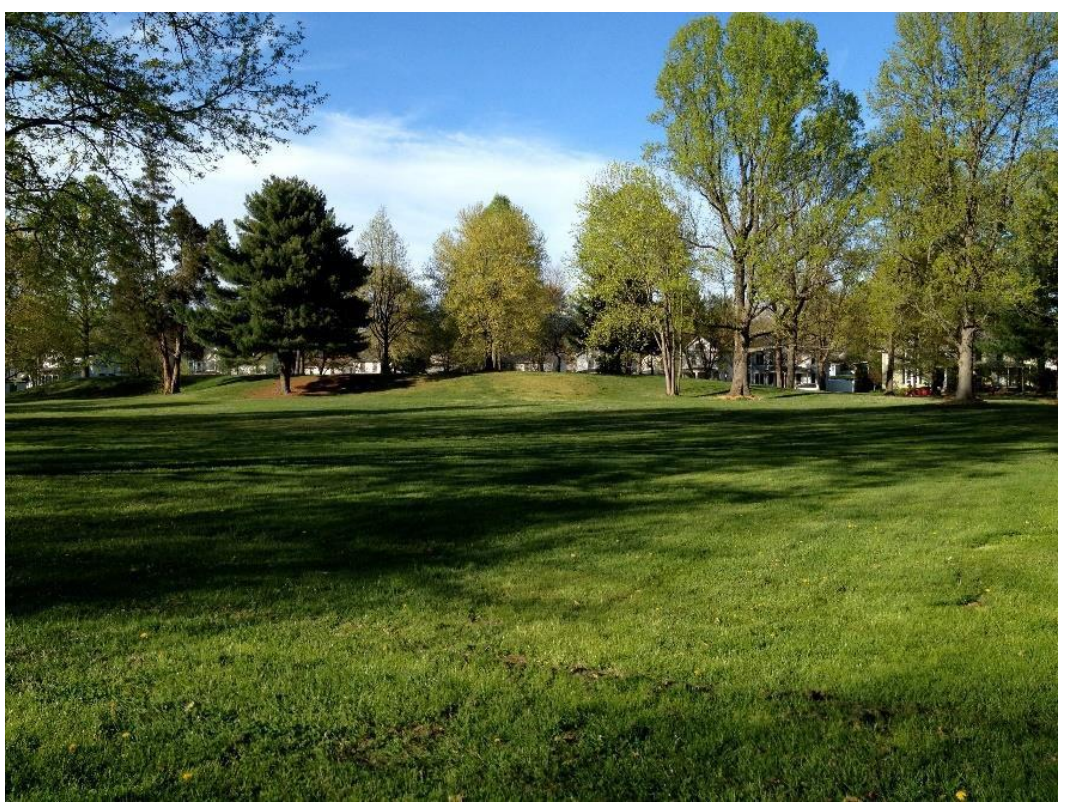

Figure 7.4. Quadranou as seen from the southwestern corner of park platform mound taking most of

the northeast corner of the park. It is one of the few areas that resembles the historic drawings of Marietta before European construction impacted the project area. Now a few trees sit on and around the mound. The mound stands at a substantial and prominent height overlooking the

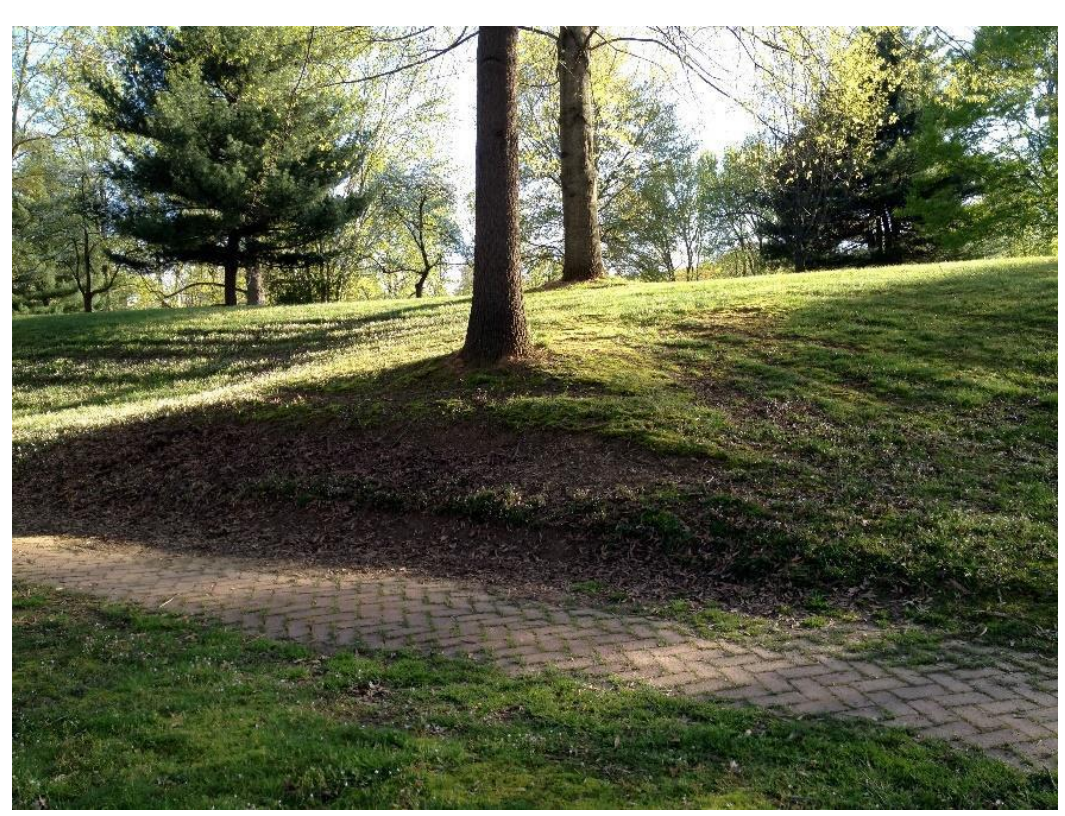

Figure 7.5. Truncation of one of the ramps leading up to Quadranou surrounding area. As I approach

I note it is level across the

platform (Figure 7.4), though

shorter on the southern corner

than the western corner due to

differences in the surrounding

topography. On each of the four

sides of the mound a ramp

protrudes from the platform.

Today, at least one of these 
ramps is truncated due to a built street and an adjacent brick sidewalk that runs across the ramp (Figure 7.5).

The mound is oriented along the Muskingum River on its widest axis. This southeast to northwest orientation is perpendicular to the Sacra Via processional, though it matches the orientation of Capitolium. I see that this orientation may not have been solely a reflection of the

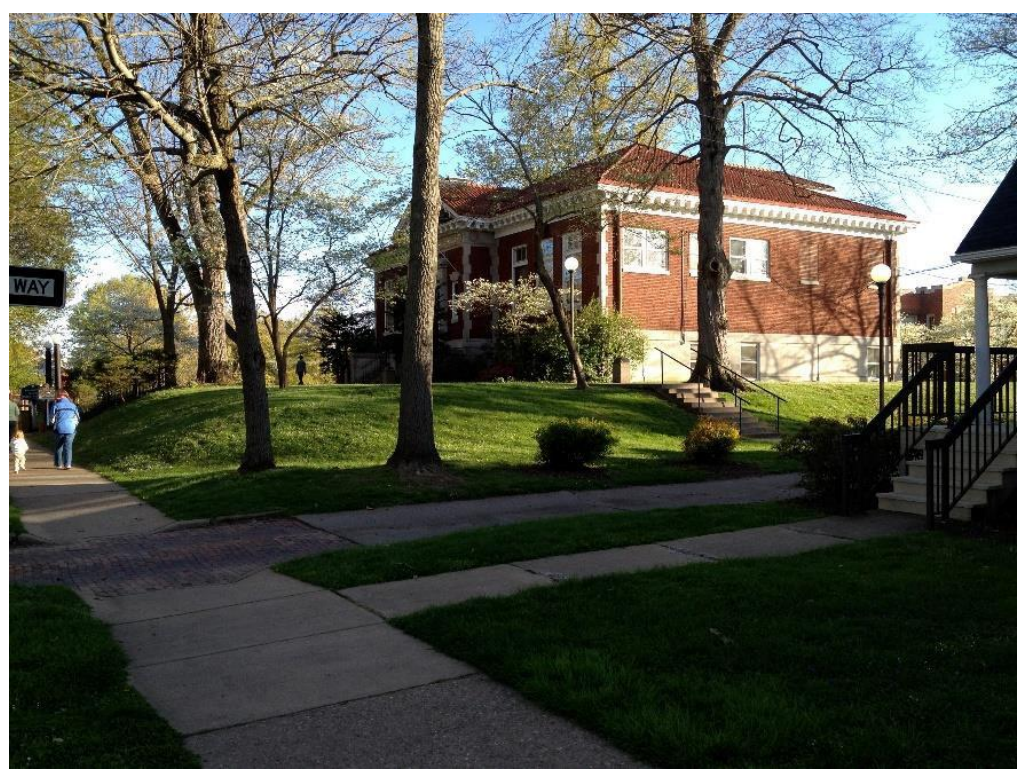

Figure 7.6. Capitolium and the historic library that sits on top natural terrain, but perhaps an alignment with the Sun at the Winter Solstice. Research has shown that there may be astronomical significance to the positioning and alignment of the mounds in Marietta, including Quadranou and Sacra Via (Romain 2005). I see that the streets of Marietta are laid out based on the orientation of the prehistoric platform mounds. Quadranou and Capitolium mounds are separated by house lined streets. Sacra Via and Quadranou are located in parks allowing for easy interaction and views between the prehistoric earthwork locations. However, as I approach Capitolium it is surrounded by, and in fact embedded within, a neighborhood that has grown up in this portion of Marietta and it is no longer possible to see Capitolium from Quadranou. Even though only a short distance separates them, the mounds now appear as separate entities in contrast to what would have been a connected relationship in the pre-European landscape within an area enclosed by a series of short walls. 
Capitolium also has ramps

leading up to the platform, though

in contrast to Quadranou, these are

only on the northeast, northwest,

and southwest sides with an

indentation cut into the southeast

side of the mound. While

Quadranou has been only slightly

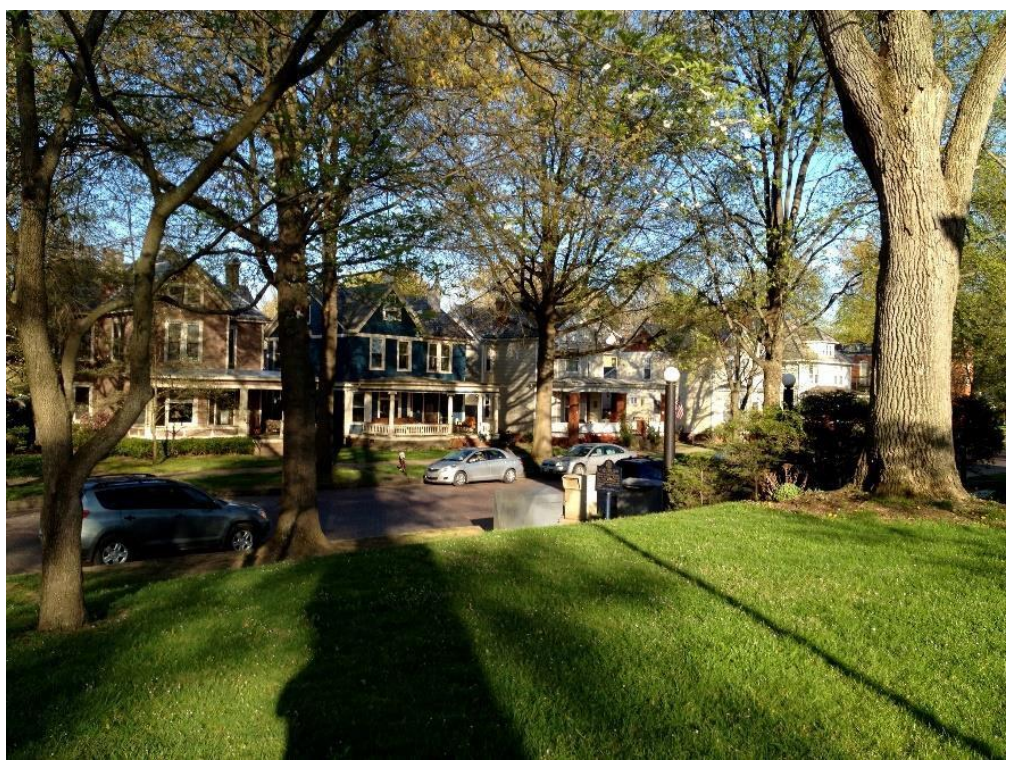

impacted by modern development,

Figure 7.7. Neighborhood of present-day Capitolium

Capitolium is now ingloriously part of the foundation of a Carnegie funded library built in the late 1800s (Figure 7.6). The library is embedded into the mound providing the building with a position of importance in the historic and modern landscape at the cost of the original mound.

Taking in the surroundings of the mound itself there is little space that has not been altered by historic development (Figure 7.7). However, having visited Quadranou, it is easier

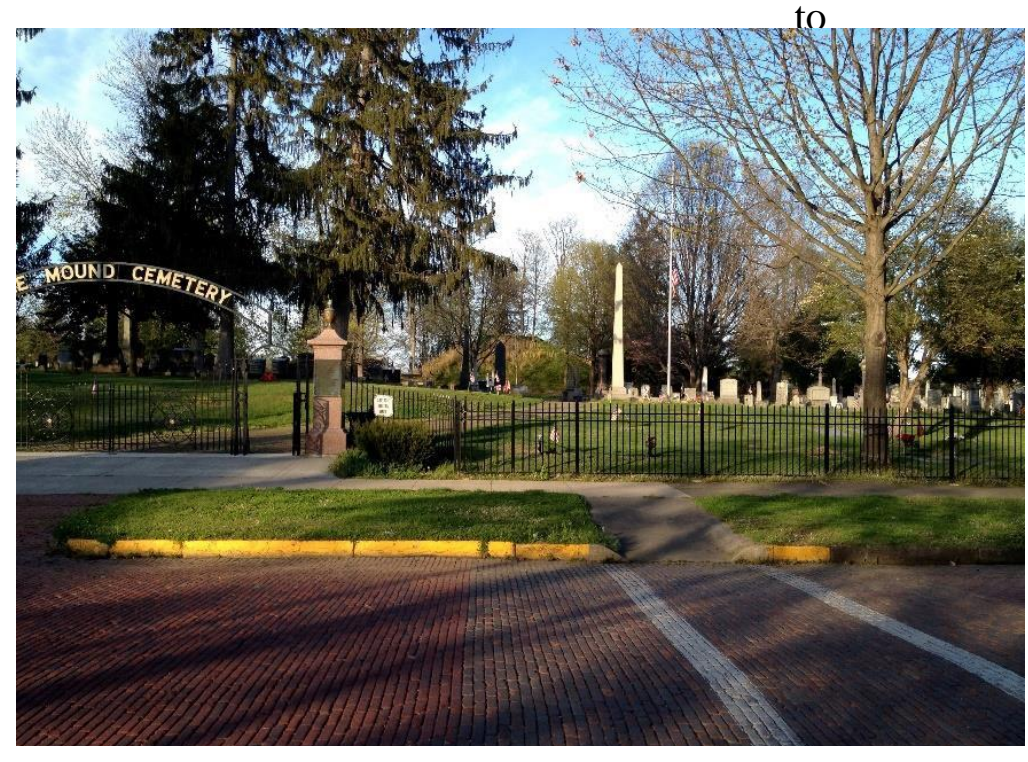

Figure 7.8. Mound Cemetery and Cemetery Mound see not only the shape of

Capitolium under its historic

construction, but to imagine the

landscape surrounding the

Hopewell mound.

I continue walking two

blocks east on Marietta's 5th Street

to arrive at Mound Cemetery, clearly named for the large conical 
mound that looms over the

headstones and monuments in

the post-contact cemetery

(Figure 7.8). This mound

extends across approximately

one third of the area of the

cemetery with a diameter of

230' including the surrounding

moat and earthen wall that

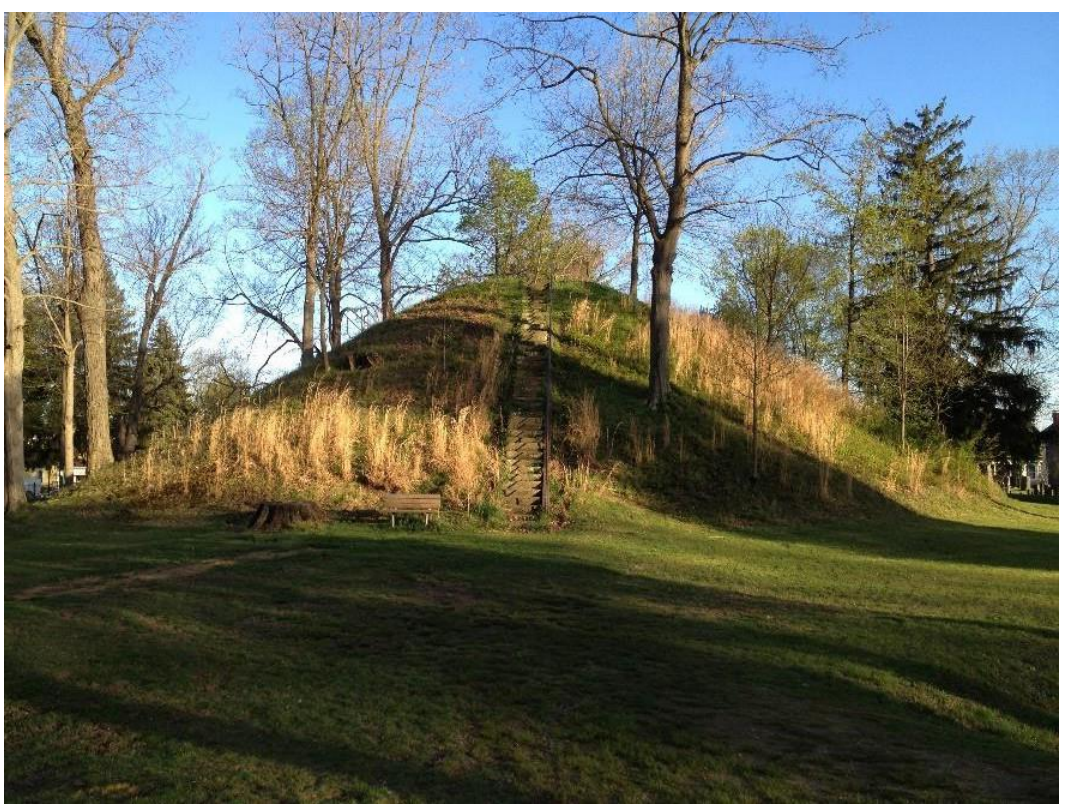

Figure 7.9. Steps placed on the side of Cemetery Mound in encircles the mound itself. the 1830s

While the historic grave stones surround the earthwork, a stairway was incongruously built in the 1830s onto the side of the mound (Cotton 1906) to tie the mound into the cemetery's historic park aesthetic and to allow visitors to view the imposing earthen structure and ascend the mound

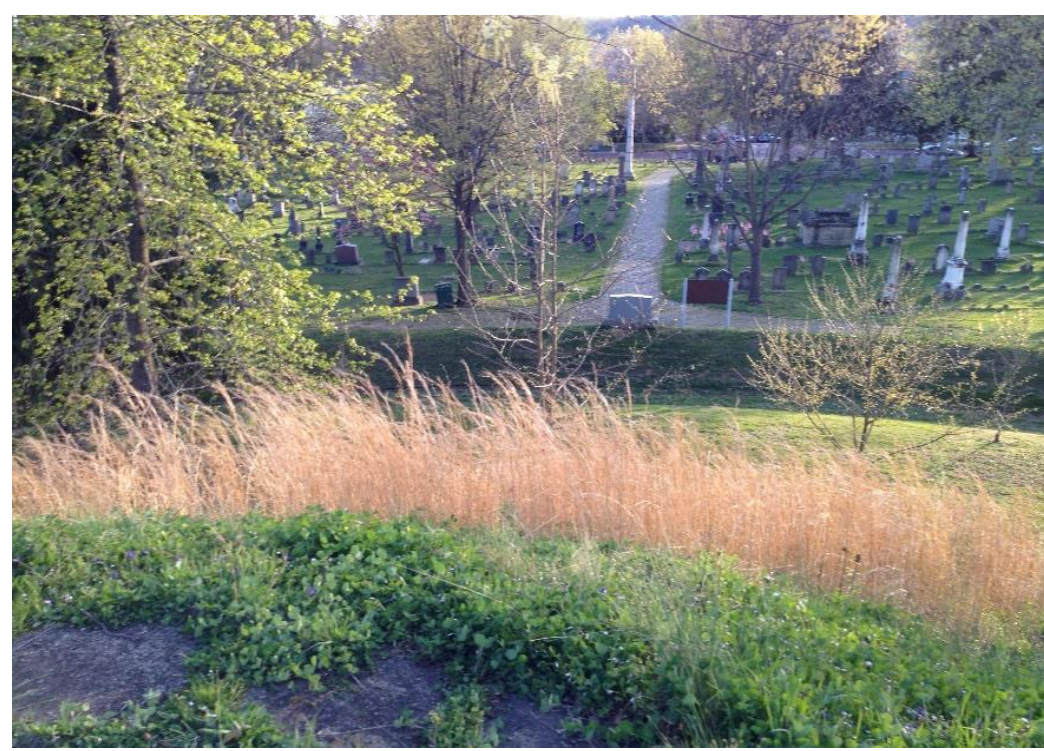

Figure 7.10. Modern view from atop Cemetery Mound looking west
(Figure 7.9). With the top of the mound truncated and leveled, pavers and benches now sit atop the mound to allow visitors to look from the top of the mound (Figure 7.10).

Questions of whether the founders of the surrounding cemetery were aware of the fact that conical Adena mounds were themselves 
earlier burial mounds cross my mind. I wonder if the linkage between the burial mound and the cemetery was a conscious, knowledgeable decision, or the result of convenience and available space. Either way, the connected funerary activities, inadvertant or intentional, give me pause to consider the less physical connections between the rehistoric and historic populations that inhabited the area.

As Cemetery Mound is the oldest of the mounds in the area, it is possible that a walk in the order described here, takes one back through time from the newer Hopewell mounds to the earlier Adena mound. While it is possible to view the surrounding landscape from atop Cemetery Mound, this was likely not a common occurrence for those who considered the area sacred. In addition, the steep sides of the mound would make climbing the mound, prior to the installation of the steps, a less than ideal activity. However, the landscape around the mound has some distinct characteristics which would have been notable even when viewed from the base of the earthwork. To the southwest a street descends from the cemetery toward the Muskingum River and provides me a partial vista as the landscape drops away from the terrace containing the cemetery and mound. However, modern foliage and structures obstruct the contemporary view of the river which was possibly open in prehistoric times. While the rivers may not be visible from the mound today, I can see the ridgeline on the opposite side of the Muskingum River. With small stone mounds recorded on the opposing bluffs there is likely a connection to the earthworks.

While the most obvious extant feature in Mound Cemetery is Cemetery Mound itself, there is also a hint in the terrain of one of the small mounds in the cemetery extending to the northwest of the mound itself. The stairs that were placed on the side of Cemetery Mound were themselves aligned to this small extension of the mound enclosure and a gap in a portion of the 
moat is level with the surrounding surface. Standing at the foot of Cemetery Mound and at the end of the small enclosure mound I see how this is the highest point before the topography dips to the north, before ascending into the surrounding mountains. The downward stepping of the terraces to the south toward the river highlights that Cemetery Mound is the highest of the mounds, though as I look back along my route I see that Capitolium sits only slightly lower on the same terrace, with Quadranou lower on the next terrace down. Sacra Via stretches down the slope from Quadranou to the river. The recent historic and modern architecture that intersect with the prehistoric earthworks play a significant role in my experience of the landscape. The area was previously an open expanse from the hillsides to the river valleys with the exception of the mounds that protruded from the landscape. However, construction has been squeezed into this flat area of Marietta and eventually up the hillsides surrounding the town.

The population of Marietta has made the earthworks their own despite, in many instances, dramatically changing the landscape to suit their needs through such features as river front parks and the building of a local college to the east of Cemetery Mound. While the historic drawings of Marietta often seem to exaggerate the earthworks to show them taking up most of the area along the rivers, in reality I see they only account for a relatively small area of the terraces that run along the eastern banks of the Muskingum River. The remnants of the prehistoric landscape appear at odds with the modern landscape. But the earthworks remain as a representation of the Adena and Hopewell societies that engaged the environment and created sacred and cultural features. The landscape, prior to European impact, necessarily provided a distinctly different cultural context from that of today's modern culture. It is perhaps the shift in context, the separation of the sacred and secular landscapes, that I find most notable. 


\section{Chapter 8 - GIS, Higuchi indices, and the modeling of landscape}

There can be no doubt that the Adena and Hopewell people left an enduring legacy in the cultural landscape of Marietta, not only in the extant mounds but through their relationship with both the built and the natural landscape in which they lived. To gain a deeper perspective and appreciation of the prehistoric landscape, a combination of approaches are implemented here that seek to combine both the egocentric and the experiential with the geocentric GIS mapped representations as outlined in previously. The use of a human centered Higuchi viewpoint embedded within GIS is implemented to aid interpretation of the Marietta landscape and the world of the mound builders.

\section{A Visualscape of prehistoric Marietta}

By experiencing and imagining the prehistoric landscape through a Higuchi viewshed places the human observer at the center of the cultural landscape analysis. The use of Higuchi indices seeks to replicate and capture, albeit in a still limited way, the egocentric and experiential world as observed and inhabited by the observer. The traditional GIS viewshed analysis (Figure 8.1) calculates only the visible parts of a viewshed as revealed based only on a terrain model and the observer's location near the base of Cemetery Mound on the west side, toward the Muskingum River. Identifying landscape intervisibility in this way is somewhat sanitized and dislocated from human scale and the human physiological aspects pursued by Higuchi involving how humans view landscapes in the context of distance, texture, color and angle of incidence. 


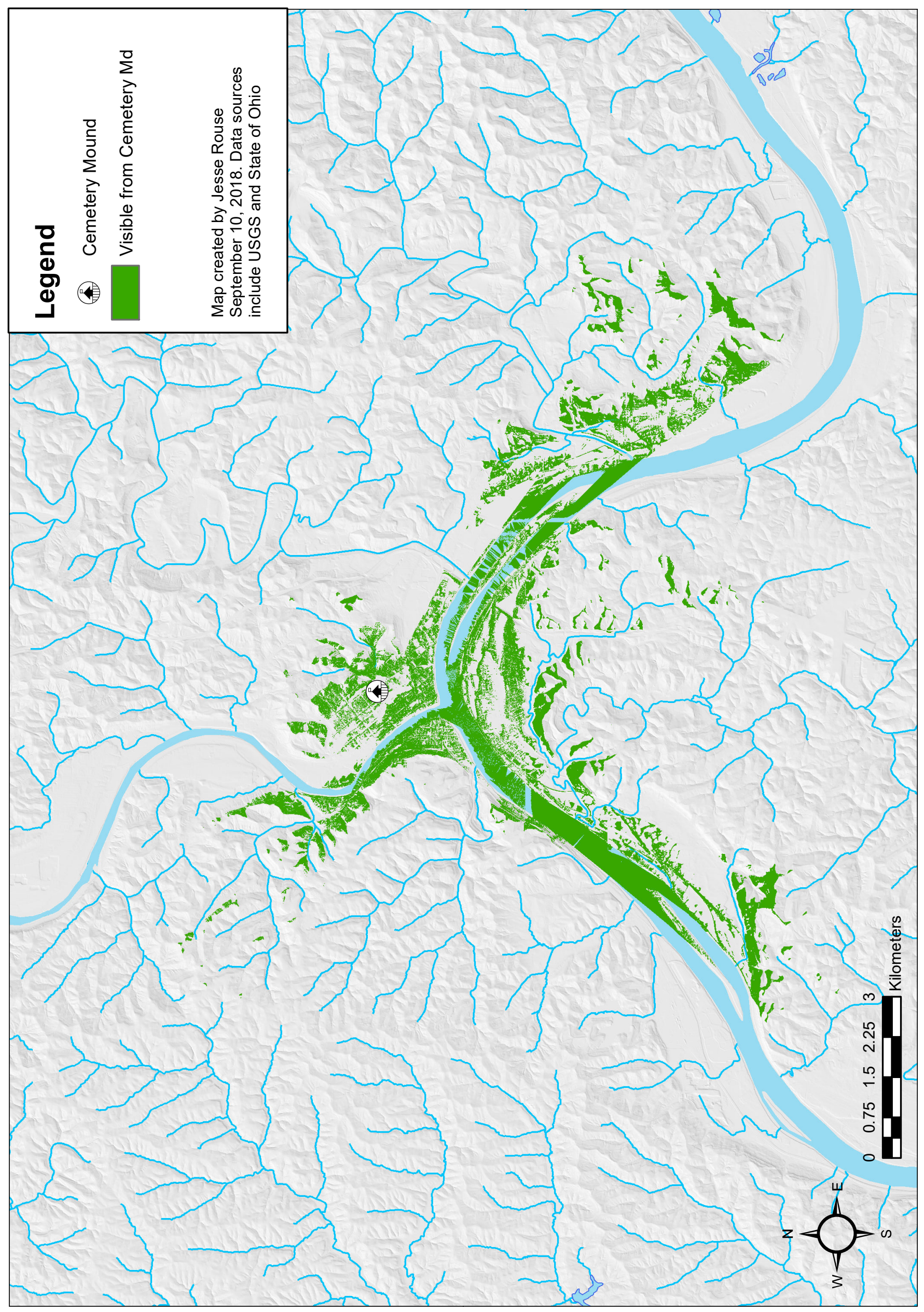

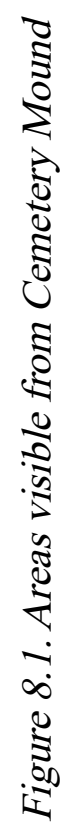




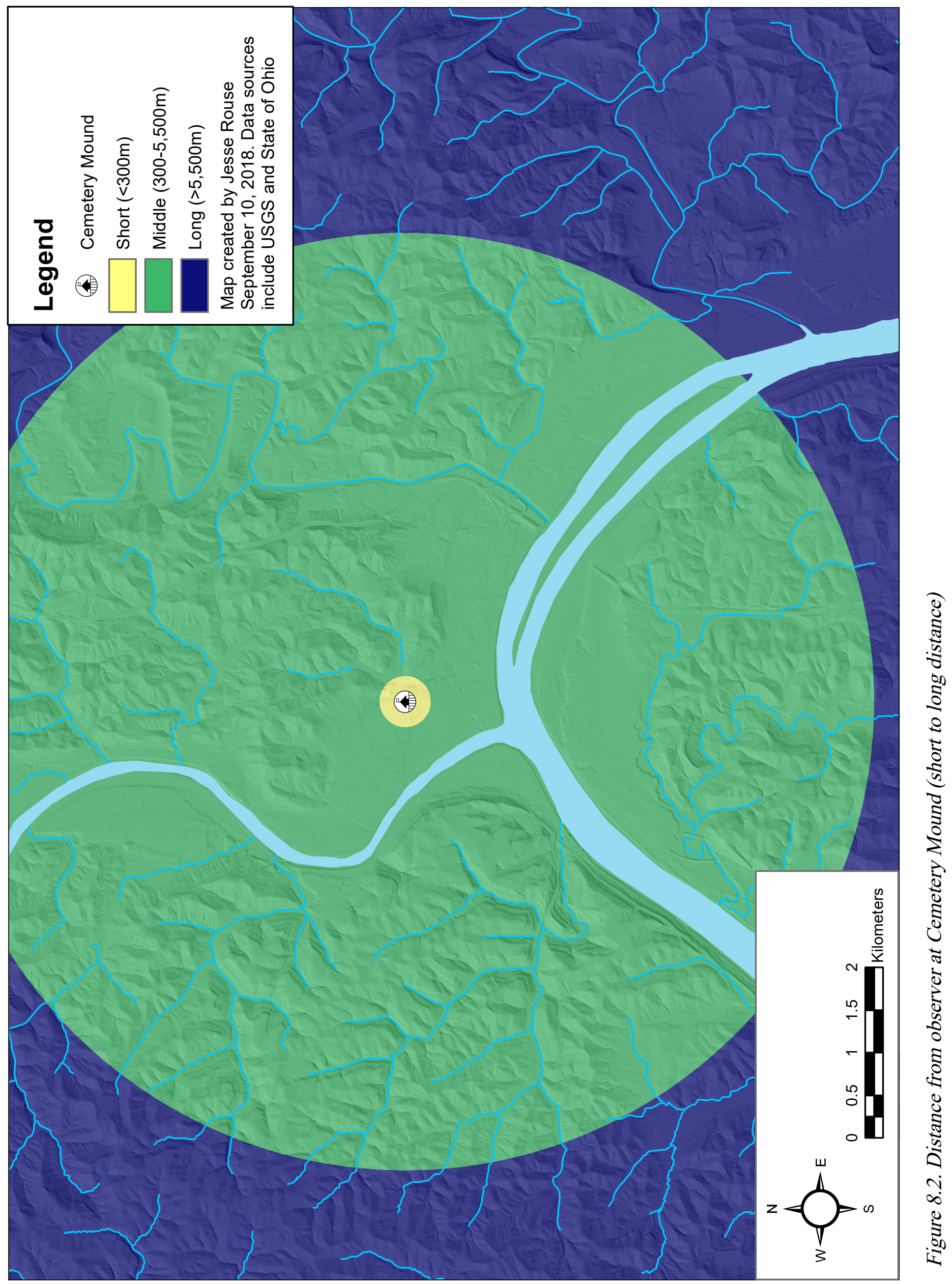


The addition of Higuchi's indices of distance, angle of depression, angle of elevation, and angle of incidence, texture, and color to the GIS derived visibility-based viewshed arguably builds a more sophisticated and nuanced representation of the observed landscape and the observer's experience of that landscape. The model developed here seeks to maintain the human observer at the heart of the analysis and to yield a landscape representation that portrays a scene stretching out from the observer. The distance index (Figure 8.2), for instance, recognizes that human sight and perception necessarily changes across distances away from the observer reflecting Higuchi's perspective of a nuanced experience of landscape and viewshed. Adjusting the relatively 'simple' visibility viewshed by accounting for the short, middle, and long range differentiation, demonstrates a considerable variation in what an observer would perceive within the viewshed. Assessing the effect of greater or lesser detail of features in a landscape potentially alters any interpretation of the modeled viewshed. Thus, when standing at Cemetery Mound looking to the west toward the Muskingum River, the details of nearby objects are relatively clear, but as the eye moves toward the river and beyond so details fade and landscape features take on more obtuse and vague forms. As the eye moves up the opposite slope, so individual trees become lost in a woodland and the skyline is broken only by large identifiable features such as houses (Figure 8.3).

\section{Adding Higuchi's}

angle of depression and angle of elevation captures (Figure 8.4) the sight lines usually seen by an observer and various features in the

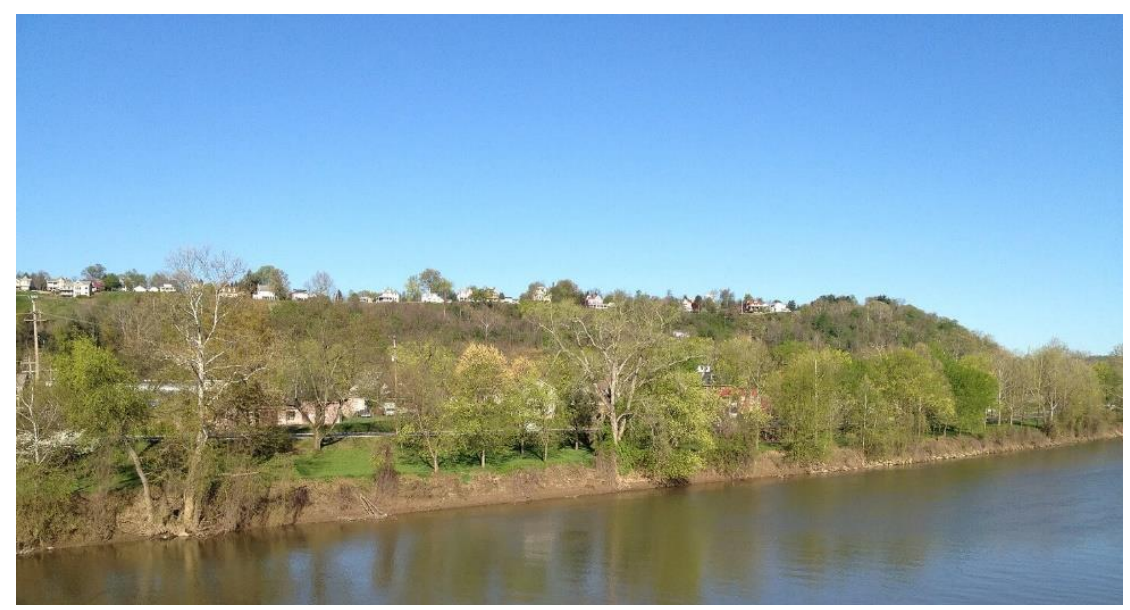

Figure 8.3. View from bridge viewing western side of Muskingum River 


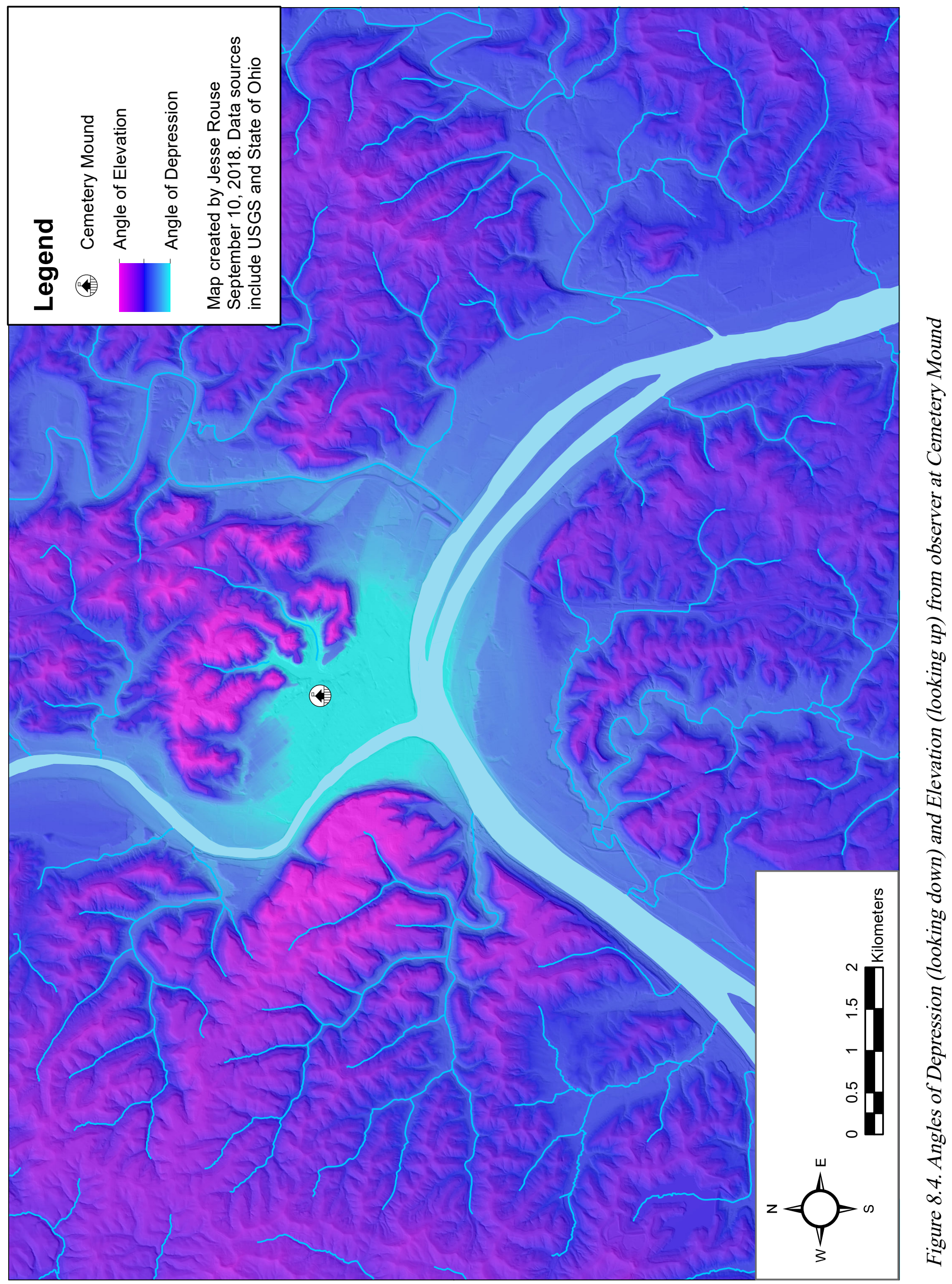


landscape. Since the tendency of humans is to visually focus within a of $55^{\circ}$ range, not all portions of a landscape are likely to be observed uniformly from any given observation location. The most common sight lines are from $25^{\circ}$ above the base line for angles of elevation to $30^{\circ}$ below for angles of depression. In addition, angles between $5^{\circ}$ and $15^{\circ}$ below the base line are the most common for human vision, with an average line of sight at an angle of depression around $10^{\circ}$ (Figure 5.5). Thus, it is easier and more comfortable for humans to view features in the landscape when looking down or up within this range of elevation and depression. Furthermore, when looking downward on features in a landscape, objects appear to be nearer thereby reinforcing the effect of distance on a viewer's acuity within a landscape (Higuchi 1988). As an observer looks upward beyond the comfort levels of elevation so features become less visually accessible and more distant resulting in a greater sense of disconnect than represented within traditional viewshed analysis. Taking the present-day view from the base of Cemetery Mound downslope to the west, an observer sees a cemetery, trees, and a grassy park sloping down to the Muskingum River. The Higuchi viewshed, building on these multiple indices, more closely captures and reflects the actual experience of a person viewing the Marietta landscape than sole reliance on the traditional line-of-sight viewshed. Accordingly, the angles of elevation and depression emphasize and de-emphasize viewable features in the landscape and are more closely related to what an observer would experience when viewing that scene in person. Consequently, the views from Cemetery Mound to the north, east, and west are all toward hills that are of greater elevation than Cemetery Mound. The enforced need for an observer to look upward to see the ridgeline surrounding the mound deemphasizes the perception of that part of the landscape and is in contrast to views to the south which are largely viewed at a downward angle 
and are seen to be more open and inviting and creates a stronger connection and appreciation similar to that experienced by the author in his physical tour of the site.

Similarly, viewing the area from the elevated position of the earthen mounds and incorporating the angles of depression and elevation generates a modeled representation that includes input closer to the experiences personally experienced in the field. Standing at the foot of a mound and looking away from the mound to the west created a personal and experiential sense of both proximity to the mound but also openness in the landscape which is again more closely mirrored in the modeled representation. Turning eastward toward the mound itself reveals a series of obstructions and steps that increase the view angle and lessens the visual impact of the mound. The built structure of the mounds, whether the relatively tall Cemetery Mound or the platform of Quadranou, occlude a large portion of the sight line and as such represents a significant obstruction of the viewshed. In the model results, these obstructions are represented by only a handful of pixels in a modeled image made up of thousands of pixels indicating considerable comparability between a personal experience of landscape and that gained through the Higuchi-GIS model. The occlusion of the sight line is also tied to the angle of incidence since facing the mounds reveals vertical frontal planes within the view. The angle of incidence deconstructs an observer's view into two primary representations, frontal planes and longitudinal planes. While the built structures create a frontal plane, the long, gentle views downslope from a mound represent a longitudinal plane (Figure 8.5). The angle of incidence thus heavily influences the sense of openness within the landscape as longitudinal planes represent stretches of open area compared to the closed nature of the frontal view where a portion of the view is blocked. 


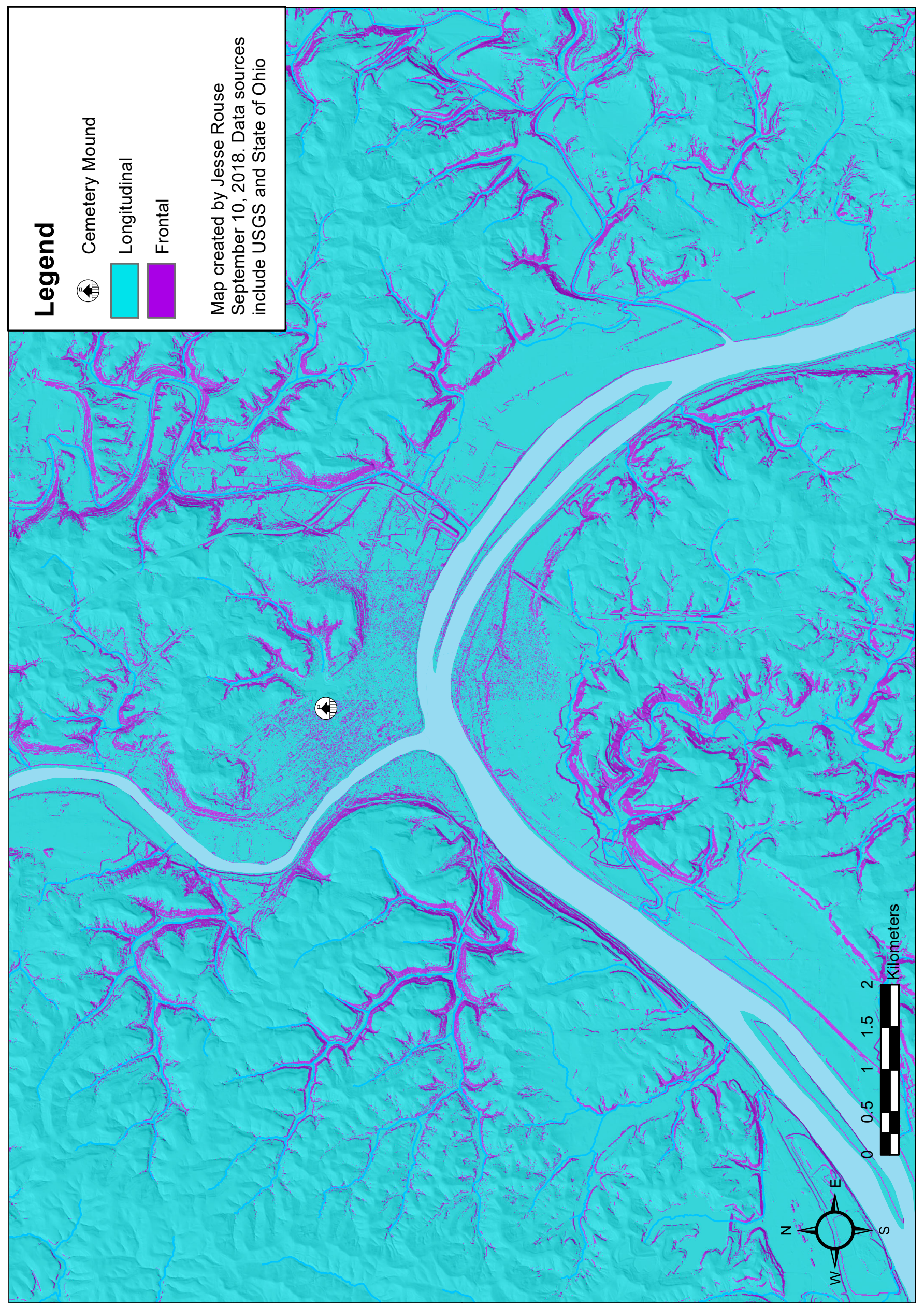

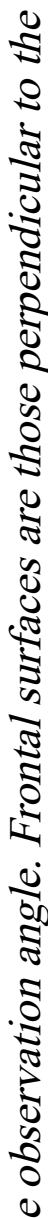

న

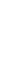

$\frac{5}{5}$

.

$\frac{2}{2}$

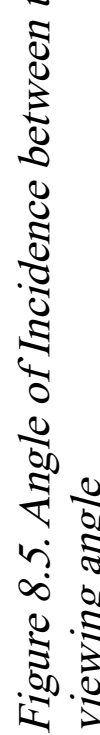


While each of the indices provides additional insight beyond the traditional measured line-of-sight viewshed, the implementation of Higuchi's composite analyses yields a summary of the disparate indices that push beyond the geocentric nature of the modeled results to embrace more egocentric responses to the landscape. The Spatial Occlusiveness composite, for instance, highlights those areas that are less comfortable for viewing and are therefore less likely to be viewed. These occluded areas in the landscape are created by combining the distance, angle of elevation, and angle of incidence indices (Figure 8.6). The portions of the project area that are within angles of depression are not represented in the results since they are not included in the composite scene and this absence leaves most of Marietta and the space around the rivers unrepresented. These results are primarily impacted by the angle of incidence and that of viewing distance. The hillsides in the project area provide some of the few frontal surfaces at the level of the observer's sight-line where the transition from angle of depression to angle of elevation. The middle and long distance indices have the greatest impact on the results and delineate those portions of the area where individual objects can be seen versus where objects are viewed as part of an aggregate feature. In areas with greater angles of elevation, such as areas with greater topographical relief or in urban areas, or even lower in the project area near the river, the angle of elevation could have a significant impact on the viewshed representation. However, given the elevation of Cemetery Mound in relation to the surrounding hills, the angle of elevation is never greater than $5^{\circ}$. The areas covered by the Spatial Occlusiveness model focus on the areas that are limiting from an egocentric perspective and thereby lessen their connection to the observer. 


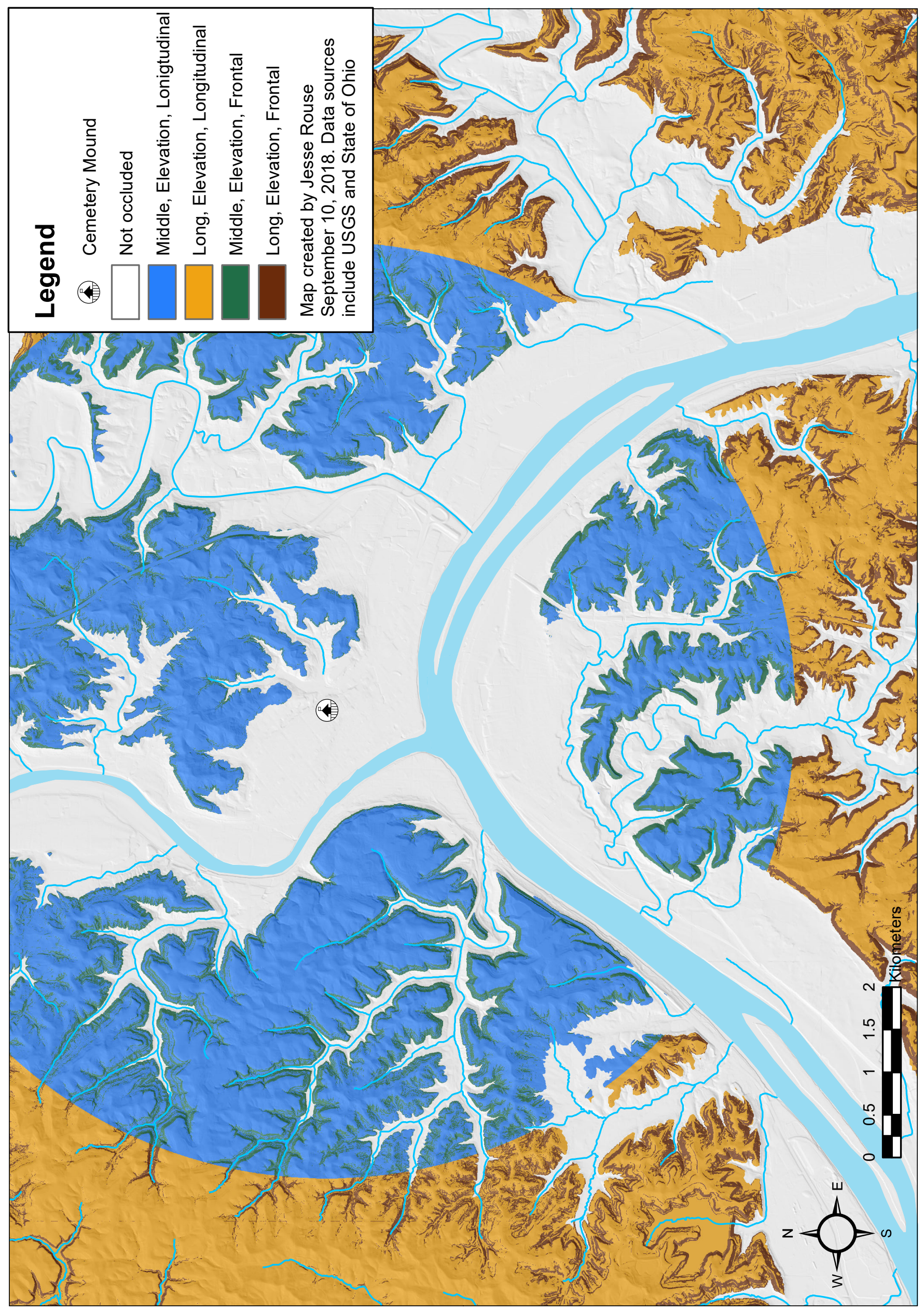

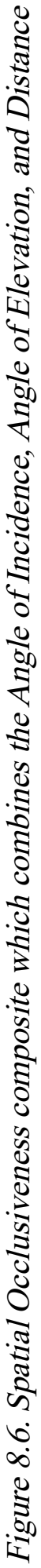


The Visibility composite adds the angle of depression and the limitation of view to the indices included in the Spatial Occlusiveness composite (Figure 8.7). For the first time, the limitations imposed by the terrain are once again made clear. The terraces and mountains surrounding an observation point at Cemetery Mound limit the visible area to a relatively small area upstream on both the Muskingum and Ohio Rivers and downstream along the Ohio River. Similar in scope to the Spatial Occlusiveness composite, within the visible area, three factors have a dominant impact on the model results: Distance, Angle of Depression or Elevation, and Angle of Incidence. Little of the surrounding area is visible from the mound particularly as distance from the mound increases. In the same way, most of the visible area is made up of longitudinal views with a small portion that has frontal views. Most of the visible area is set within an angle of depression leaving most of the area in a personally comfortable viewing angle from the observation point.

It is suggested here that blending the egocentric perspective provided by these five visual indices within the GIS geocentric landscape provides a more human-centered representation of landscape as experienced by those physically in the landscape and certainly more so than that provided by the traditional intervisibility models. To operationalize such an approach and to bring the modeled landscape viewshed closer to a personal experience of landscape one could consider moving through a landscape within the model as if encapsulated within a Higuchi 'bubble' where the viewshed is modeled on the fly. Such a model is similar in many ways to physically walking and experiencing Marietta and allowing the model to recalibrate as the landscape is traversed allowing those unable to visit a site to gain a deeper understanding of the visibility of features within the landscape. 


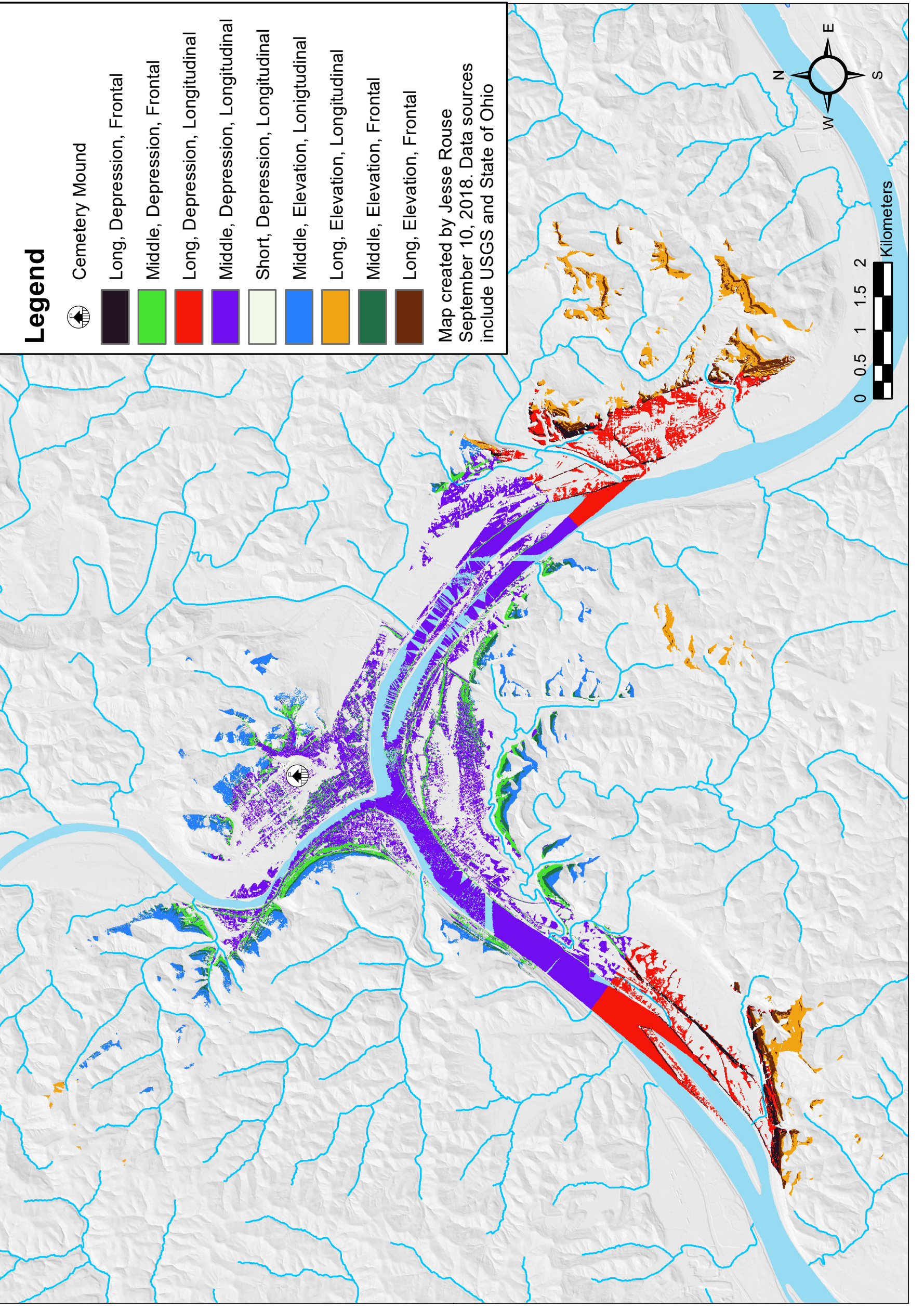

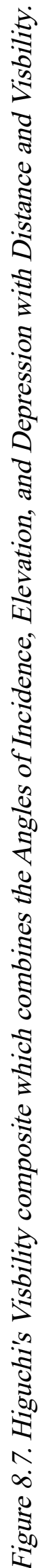




\section{Prospect, refuge, and 'scapes}

Interestingly, these indices (distance, depression, elevation, and incidence) relate closely to Appleton's (1975) discussion of prospect and refuge and reinforce the consideration of the physiological role of Higuchi's indices as a means to explore affordances in the landscape. The sense of opportunity that prospect engenders within the experience of the landscape is tied to open views such as those measured by longitudinal planes and an angle of depression. As much of the area throughout modern Marietta lies below Cemetery Mound, the position of the mound clearly reflects a strong sense of prospect within the landscape. Before the historic construction that brought buildings and streets to the project area, the open views down to the terraces of the Muskingum and Ohio Rivers would have provided just such a sense of prospect. Today, from the Quadranou Mound down the Sacra Via to the Muskingum River, such a view and sense of prospect can still be experienced.

However, there were many areas in the landscape that were limited in view or visibility and which created a sense of refuge for the mound builders. The sense of refuge created by the frontal planes and angles of elevation limit not only what the observer can perceive but what others can see of the observer. To return to an earlier example, the area of invisibility that was created behind Cemetery Mound provides an obscured position from those observing the mound from the Muskingum River creating a position of refuge within the area of the mounds. This example of refuge could similarly be transferred to the whole of the Marietta Mounds given their position between higher peaks on the north, east, and west providing occlusion from viewpoints from most locations surrounding the mounds. In addition, the bends on both the Muskingum and Ohio Rivers just before Marietta would have limited observation of the mounds until the last 
moment when what must have been an impressive prospect of the Marietta mounds came into view.

Marietta's potential as refuge is not to suggest that the site of the mounds was intended to be hidden, but to suggest that the physiological experience and physiographic nature of the area bring about a unique sense of place. In reality, the role of prospect is equally observable in the Marietta Mounds. Given the nature of the local topography, it is easy to see how, from the south and from the Muskingum River, people traveling to the site of the mounds were presented with the mounds from their observation points. The mounds are nestled along the upward sight line that would have been forced by the hilly terrain. In addition, the bends in the river may have created 'a reveal' as travelers on the rivers would have come into sight of the terraces creating a break in the higher hills on the north side of the Ohio and the east side of the Muskingum.

Building on Higuchi's indices and Appleton's consideration of the psychological/cultural response to landscape it is possible to link the visualscape to other 'scapes as suggested earlier. Appleton's prospect and refuge offers insight into taskscapes for while not linked to a specific locale as in Ingold's description of dwelling, the perception of prospect and refuge closely relates as to how individuals experience the landscape. While the measured angles of the indices cannot reflect the egocentric intent of those in the landscape, the model can provide a geocentric representation of potential of prospect and refuge within the landscape. It is suggested here that blending the egocentric perspective provided by the description of experience with the visual indices modeled in GIS creates a geocentric representation that provides a more human-centered depiction of landscape such as experienced by those physically in the landscape and certainly more than that provided by traditional viewshed models. 


\section{Chapter 9 - Conclusion and future directions}

Going through the litany of Higuchi indices it is possible to lose sight of the ultimate goal of this study and again become detached from the landscape itself. It is argued here that the traditional mapping of viewsheds is relatively crude and fails to capture the qualitative essence of a landscape as experienced in person. Higuchi's approach proceeds further in capturing more of the experience of landscape through its physiological and human-centric focus. The consideration of the various 'scapes provides a depiction of the space, but it is the experiential that links an individual to a place. By connecting the egocentric consideration of Higuchi's approach and the geocentric world of GIS landscape modeling, this study seeks to lessen the dichotomy of space and place and to bring together the experiential and the geospatial, the egocentric and geocentric, and the qualitative and the quantitative. Tilley's (1994) reference to the 'affinity' that one has with a landscape, and with the connection to a place, implies that each landscape is encultured not only through our experience of the landscape, but by our very observation and consideration of that landscape.

The role of space and place in understanding human connectedness to a cultural landscape is directly linked to expanding our personal experience and consideration of the cultural nature of an area. As suggested, resourcescapes and taskscapes offer a tentative view into the material and active lives of prehistoric people. Similarly, visualscapes offer a degree of separation from the landscape not available as we view our surroundings and which clarifies the spatial configurations in the landscape such as the relative position of the Marietta mounds, their location in the river valleys, and the location of the Marietta mound group in relation to other mound groups. What is viewed in the landscape drives cultural meaning and connectivity, but the 
landscape viewshed as assessed through the GIS modeled Higuchi approach yields a broader perspective that connects a human-centered viewshed to the material landscape and as a basis for examining the cultural landscape.

Between the model and the experience of the extant prehistoric features in the Marietta landscape there are affordances offered by the landscape and the features that are teased out through the two previous chapters. For instance, Sacra Via's long upward incline likely drew the eyes of visitors as they entered the Muskingum River, much as they did in this author's walk, offering potential and prospect for those coming to visit the site. At the same time, once atop the terrace and the mounds, there is a degree of refuge in being near these substantive earthworks. These affordances within the landscape are a culmination of the material landscape and the symbolism inherent in the extant cultural landscape. The connection to the symbolic landscape would have been bodily impacted by the bounding nature of the associated embankments. However, the two embankment areas, one encircling the Hopewell platform mounds and a smaller one enclosing the Adena Cemetery Mound, would have provided the observer with a delineation within the landscape and perhaps importantly, a separation in meaning of the mound features.

In looking at the prehistoric landscape that is now embedded in Marietta, Ohio, the goal of this study has been to join the use of quantitative and qualitative methods to gain insight into the past cultural landscape. In this case, it is the joining of a phenomenological approach and GIS driven models of landscape that provides a combined perspective of the area based on egocentric and geocentric perspectives. This study represents a meeting point, a bridge, a link, between currently popular and traditional viewshed models used in landscape archaeology and an experiential representation of landscape. The 'phenomenological walk' and the modeled 
representation of the landscape are two parts of the same discourse, each bringing strengths and weaknesses but which when combined through Higuchi viewsheds complement each other.

The move from a spatial assessment toward a platial assessment of viewshed within the archaeological record represents a move toward Tilley's (2004) call for greater emphasis on the social and cultural geography of place. The use of Higuchi viewsheds provides a degree of replicability in generating a human-centered viewshed and given that the model parameters can be altered, the model can be adjusted to the local context. Studying cultural landscapes using geospatial technologies thus creates a duality of perspective: the perspective of the observer leading to the experience of landscape and the perspective captured through the data and visualization and mapping of spatial information. The egocentric perspective is conceptually derived from the subjective view of the individual. Based on personal experience and cognitive links to the body of the observer, the egocentric view is situated in the model. The experience of viewing surrounding landforms, objects, and modeling those aspects through Higuchi indices links the viewer to the landscape, immersing them, and situating them in the world. The cognitive connection between the body and the world allows the observer to connect with, and experience, their surroundings. The observer cannot assume to ever describe the world in specific detail, necessarily leaving a level of ambiguity, or abstraction, in the recounting of their experience.

Conversely, the geocentric perspective is based on a presupposition of knowledge of an area and a spatial representation of key information in the form of a map, aerial or satellite image, or a database of terrain information. Based on a priori knowledge and from an overview perspective provided through the map, the geocentric viewpoint lies outside the immediacy of a personal experience of landscape and is global in scope. The holistic perspective of a GIS exemplifies the external nature of the geocentric perspective that assumes the observer has 
significant information about the landscape. However, in placing the observer above the landscape this perspective loses sight of the actual experience and qualities of that landscape Ideally, just as an egocentric perspective does not preclude prior knowledge of an area, a geocentric perspective should not exclude the observer's personal experience of a landscape. When considering a relict landscape, it is challenging to integrate the personal experiences of the modern landscape and the ideas it creates in the mind of the landscape archaeologist with the computational modeling of the spatial information.

The combinatorial model proposed here, like the photographs, recordings, and other depictions of the phenomenological walk, represents a reflection. But a reflection on information and data that is beyond what our senses can perceive at any one time. It is a perspective that builds upon and expands beyond the individualistic view of the observer. The result is a step toward bridging the qualitative and phenomenological experience to that of spatial analysis. Both experiential and modeled approaches often leave their results at the level of description, only implicitly suggesting an interpretation of the landscape activities. There are those (Spector 1993, Brenneman 1989) who have presented their field and analytical results as a story of interpretation, weaving people's lives and interactions into an imagined narrative set within the landscape. Others (Bradley 1998, Gillings 2015) suggest that affordances found in the observation of a landscape are part of the experience of landscape and allow for others to create their own interpretations.

In phenomenology, the bracketed description is often the final product. In a modeled landscape the map is the key. However, in a Higuchi approach, especially one in the digital era, the fact that these examples stop short of a reductionist and explicit interpretation may be for the best as it leaves the vital act of interpretation to the reader not to the algorithm. 


\section{Bibliography}

Aitken, Stuart, and Jim Craine. 2009. "Into the Image and Beyond: Affective Visual Geographies and GIScience." In Qualitative GIS: A Mixed Methods Approach, edited by Meghan Cope and Sarah Elwood, 139-155. Thousand Oaks, CA: Sage Publications.

Aldenderfer, Mark S., and Herbert D. G. Maschner. 1996. Anthropology, space, and geographic information systems, Spatial information systems. New York: Oxford University Press.

Allen, Kathleen M. S., Stanton W. Green, and Ezra B. W. Zubrow. 1990. Interpreting space: GIS and archaeology, Applications of geographic information systems. New York: Taylor \& Francis.

Appleton, Jay. 1975. The Experience of Landscape. New York: Wiley.

Ashmore, Wendy, and Arthur Bernard Knapp, eds. 1999. Archaeologies of Landscape: Contemporary Perspectives. Malden, MA: Blackwell.

Aston, Michael, and Trevor Rowley. 1974. Landscape archaeology: an introduction to fieldwork techniques on post-Roman landscapes. Newton Abbot: David \& Charles.

Barnes, Trevor J., and James S. Duncan. 1992. Writing worlds: discourse, text, and metaphor in the representation of landscape. New York: Routledge.

Batty, Michael. 2001. "Exploring isovist fields: space and shape in architectural and urban morphology." Environment and Planning B: Planning and Design 28 (1): 123-50.

Bell, David Andrew. 1990. Husserl. New York: Routledge.

Bender, Barbara. 1999. Stonehenge: Making Space. New York: Berg.

Benedikt, M. L. 1979. "To Take Hold of Space: isovists and isovist fields." Environment and Planning B 6 (1): 47-65.

Bergeron, Susan J. 2004. "Toward a phenomenological approach to prehistoric cultural landscape analysis: The Marietta Mounds Internet GIS." MA, Geography, West Virginia University.

Bergeron, Susan J. 2011. "Engaging the virtual landscape: toward an experiential approach to historical place analysis through a Spatial Experience Engine." PhD, Geography, West Virginia University. 
Binford, Lewis R. 1977. "General Introduction." In For Theory Building in Archaeology: Essays on Faunal Remains, Aquatic Resources, Spatial Analyses, and Systemic Modeling, edited by Lewis R. Binford, 1-10. New York: Academic Press.

Bodenhamer, David J., John Corrigan, and Trevor M. Harris, eds. 2010. The spatial humanities: GIS and the future of humanities scholarship. Indianapolis: Indiana University Press.

Boschmann, E. Eric, and Emily Cubbon. 2014. "Sketch Maps and Qualitative GIS: Using Cartographies of Individual Spatial Narratives in Geographic Research." The Professional Geographer 66 (2): 236-248.

Brabyn, Lars. 1996. "Solutions for characterising natural landscapes in New Zealand using geographical information systems." Journal of Environmental Management 76 (1): 23- 34.

Bradley, Bruce, and Dennis Stanford. 2004. "The North Atlantic ice-edge corridor: a possible Palaeolithic route to the New World." World Archaeology 36 (4): 459-478.

Bradley, Richard. 1998. The significance of monuments: on the shaping of human experience in Neolithic and Bronze Age Europe. New York: Routledge.

Brenneman, William L. Jr. 1989. "The Circle and the Cross: Loric and sacred space in the holy wells of Ireland." In Dwelling, Place \& Environment, edited by David Seamon and Robert Mugerauer, 137-158. New York: Columbia University Press.

Brown, A. G. 2003. "Time, Space and Causality in Floodplain Archaeology." In Alluvial Archaeology in Europe: proceedings of the Alluvial Archaeology of North-West Europe and Mediterranean, edited by Andrew J. Howard, Mark G. Macklin, David Glynn Passmore and D. G. Passmore, 15-24. Exton, PA: Taylor and Francis.

Bryant, William C. and Sydney H. Gay. 1883. A Popular History of the United States: From the First Discovery of the Western Hemisphere by the Northmen, to the End of the Civil War. Preceded by a Sketch of the Prehistoric Period and the Age of the Mound Builders, Volume 1. New York: Scribner and Sons.

Burgess, Stephen, and Scott Orford. 2010. "Qualitative GIS: combining theory and method." NCRM Research Methods Festival 2010, St. Catherine's College, Oxford. Burrough, P. A., and Rachael McDonnell. 1998. Principles of geographical information systems for land resources assessment. New York: Oxford University Press. 
Canada's Historic Places. 2010. Standards and Guidelines for the Conservation of Historic Places in Canada. $2^{\text {nd }}$ edition. https://www.historicplaces.ca/media/18072/81468-parks-s+geng-web2.pdf (last accessed 10 September 2018)

Carr, David. 1987. Interpreting Husserl: Critical and Comparative Studies. Hingham: Kluwer. Chorley, Richard J., and Peter Haggett. 1967. Models in geography. London: Methuen.

Christopherson, Gary. 2003. "Using ARC/GRID to Calculate Topographic Prominence in an Archaeological Landscape." Proceedings of the 2003 ESRI International User Conference, San Diego, CA: ESRI.

Claassen, Cheryl. 1998. Shells, Cambridge Manuals in Archaeology. New York: Cambridge University Press.

Clarke, David L. 1972. Models in Archaeology. London: Methuen.

Cooper, David, and Ian Gregory. 2011. "Mapping the English Lake District: a literary GIS." Transactions of the Institute of British Geographers 36: 89-108.

Cope, Meghan, and Sarah Elwood. 2009. Qualitative GIS: a mixed methods approach. Los Angeles: Sage.

Cosgrove, Denis. 2008. Geography and Vision: Seeing, Imagining and Representing the World. New York: I. B. Tauris.

Cosgrove, Denis E. 1998. Social formation and symbolic landscape. Madison, WI: The University of Wisconsin Press.

Cotton, Willia Dawson. 1906. Sketch of Mound cemetery, Marietta, Ohio. Marietta, OH: Marietta Register.

Craig, Willam J., Trevor M. Harris, and Daniel Weiner, eds. 2002. Community Participation and Geographic Information Systems. New York: Taylor and Francis.

Crowell, Steven, and Jeff Malpas, eds. 2007. Transcendental Heidegger. Stanford: Stanford University Press.

Dant, Tim. 2005. Materiality and Society. New York: Open University Press.

Darby, H. C. 1973. A new historical geography of England. Cambridge: Cambridge University Press. 
David, Bruno, and Julian Thomas. 2008. Handbook of landscape archaeology, World Archaeological Congress research handbooks in archaeology. Walnut Creek, CA: Left Coast Press.

Davis, Larry S., and Michael L. Benedikt. 1979. "Computational models of space: Isovists and isovist fields." Computer Graphics and Image Processing 11 (1):49-72.

DeMers, Michael N. 1997. Fundamentals of geographic information systems. New York: Wiley.

Dodge, Martin, Mary McDerby, and Martin Turner. 2008. Geographic visualization: concepts, tools and applications. Hoboken, NJ: Wiley.

Ehrhardt, Kathleen L. 2009. "Copper Working Technologies, Contexts of Use, and Social Complexity in the Eastern Woodlands of Native North America." Journal of World Prehistory 22 (3): 213-235.

Elwood, Sarah. 2006. "Critical Issues in Participatory GIS: Deconstructions, Reconstructions, and New Research Directions." Transactions in GIS 10 (5): 693-708.

Epstein, Joshua M., and Robert Axtell. 1996. Growing Artificial Societies: Social Science from the Bottom Up. Washington, DC: The Brookings Institute.

Fish, Peter F. 1996. "Probable and fuzzy models of the viewshed operation." In Innovations in GIS: selected papers from the first National Conference on GIS Research UK, edited by Michael Worboys, 161-175. Bristol, PA: Taylor \& Francis.

Fleming, Andrew. 1999. "Phenomenology and the Megaliths of Wales: a Dreaming Too Far?" Oxford Journal of Archaeology 18 (2): 119-125.

Forte, Maurizio. 2000. "Archaeology and virtual micro-topography: the creation of DEMs for reconstructing fossil landscapes by Remote Sensing and GIS applications." In Beyond the Map: Archaeology and Spatial Technologies, edited by Gary Lock, 199-213. Washinton, DC: IOS Press.

Gerber, N'omi. 2008. "Marietta, Ohio: Washington County, Eastern Ohio: Early Historic Period Steps to Preserve Earthworks." In Archaeology in America: An Encyclopedia, edited by Linda S. Cordell, Kent Lightfoot, Francis McManamon and George Milner, 103-4. Westport, CT: Greenwood Publishing Group.

Gibson, James Jerome. 1950. The perception of the visual world. Boston: Houghton Mifflin. 
Gillings, Mark, and Glyn Gooodrick. 1996. "Sensuous and reflexive GIS: exploring visualisation and VRML." Internet Archaeology 1. http://intarch.ac.uk/journal/issue1/gillings_index.html (last accessed 10 September 2018)

Gillings, Mark. 2012 “Landscape Phenomenology, GIS and the role of affordance.” Journal of Archaeological Method and Theory 19: 601-611.

Goad, Sharon I. 1979. "Middle Woodland Exchange in the Prehistoric Southeastern United States." In Hopewell Archaeology: The Chillicothe Conference, edited by David S. Brose and N'omi Gerber, 237-246. Kent, OH: Kent State University Press.

Goodchild, Michael. 1992. "Geographical Information Science." International Journal of Geographical Information Systems. 6 (1): 31-45.

Goodchild, Michael F., and Donald G. Janelle. 2004. Spatially integrated social science. New York: Oxford University Press.

Google Earth 6.0. Google, Mountainview, CA.

Gremillion, Kristen J. 2004. "Seed Processing and the Origins of Food Production in Eastern North America." American Antiquity 69 (2): 215-233.

Harris, Trevor M. 2008. "Scale as Artifact: GIS, Ecological Fallacy, and Archaeological Analysis." In Confronting Scale in Archaeology: Issues of Theory and Practice, edited by Gary Lock and Brian Leigh Molyneaux, 39-54. Vermillion, SD: Springer.

Harris, Trevor M. 2016. “From PGIS to Participatory Deep Mapping and Spatial Storytelling: An Evolving Trajectory in Community Knowledge Representation in GIS” The Cartographic Journal 53(4): 318-325.

Harris, Trevor M., and Gary R. Lock. 1995. "Toward an Evaluation of GIS in European Archaeology: the past, present and future of theory and applications." In Archaeology and Geographic Information Systems: A European Perspective, edited by Gary R. Lock and Zoran Stancic, 349-366. Bristol, PA: Taylor and Francis.

Harris, Trevor M., and L. Jesse Rouse. 2004. "GIS and Society perspectives on GIScience in archaeology." Society for American Archaeology, Montreal.

Harris, Trevor M., Briane K. Turley, and L. Jesse Rouse. 2000. "Integrating the humanities and geospatial science: exploring cultural resources and sacred space through Internet GIS." Electronic Cultural Atlas Initiative, University of California, Berkeley. 
Harris, Trevor M., and Daniel Weiner. 1996. "GIS and Society: The Social Implications of How People, Space, and Environment Are Represented in GIS." Scientific Report for the Initiative 19 Specialist Meeting, South Haven, MN, March 2-5.

Hegel, G. W. F. 1931. The Phenomenology of Mind. Edited by J. B. Baillie. New York: The MacMillan Company.

Heidegger, Martin. 1962. Being in Time. Malden, MA: Blackwell Publishing.

Higuchi, T. 1988. Visual and spatial structure of landscapes. Cambridge, MA: MIT Press.

Hodder, Ian. 1987. "Contextual archaeology: an interpretation of Catal Hüyük and a discussion of the origins of agriculture." Bulletin of the Institute of Archaeology 24: 43-56.

Hodder, Ian. 2003. Reading the past: current approaches to interpretation in archaeology. Cambridge: Cambridge University Press.

Holcomb, Derrold W. 1992. "Field Report: Shuttle Imaging Radar and Archaeological Survey in China's Taklamakan Desert." Journal of Field Archaeology 19 (1): 129-138.

Hoskins, William George. 1970. The making of the English landscape. Baltimore, MD: Penguin Books.

Ingold, Tim. 1993. "The temporality of the landscape." World Archaeology 25 (2): 152-174.

Johnson, Matthew. 2006. Ideas of Landscape. Malden, MA: Wiley-Blackwell.

Johnston, Ronald John. 1994. "Carl Sauer." In Dictionary of Human Geography, edited by Ronald John Johnston, Derek Gregory and David Marshall Smith, 579-581. Malden, MA: Blackwell.

Jung, Jin-Kyu. 2009. "Computer-Aided Qualitative GIS: A Software-level Integration of Qualitative Research and GIS." In Qualitative GIS: A Mixed Methods Approach, edited by Meghan Cope and Sarah Elwood, 115-136. Thousand Oaks, CA: Sage Publications.

Knappett, Carl. 2005. Thinking through Material Culture: An Interdisciplinary Perspective. Philadelphia: University of Pennsylvania Press.

Korp, Maureen. 1990. The sacred geography of the American mound builders, Native American studies. Lewiston, NY: Edwin Mellen Press.

Kroeber, A. L. 1934. Uto-Aztecan Languages of Mexico. Vol. 8, Ibero-Americana. Berkeley: University of California Press. 
Kvamme, Kenneth. 1990. "One-sample tests in regional archaeological analysis: new possibilities through computer technology." American Antiquity 55 (2): 367-381.

Kvamme, Kenneth L. 1996. "Investigating Chipping Debris Scatters: GIS As an Analytical Engine." In New Methods, Old Problems: Geographic Information Systems in Modern Archaeological Research, edited by Herbert D. G. Maschner, 38-74. Carbondale: Southern Illinois University at Carbondale.

Kwan, Mei Po, and LaDonna Knigge. 2006. "Doing Qualitative Research Using GIS: an oxymoronic endeavor?" Environment and Planning A 38 (11): 1999-2002.

Kwan, Mei-Po, and Stuart Aitken. 2010. "GIS as Qualitative Research: Knowledge, Participatory Politics and Cartographies of Affect." In The Sage Handbook of Qualitative Geography, edited by Dydia DeLyser and Steve Herbert, 287-304. Thousand Oaks, CA: Sage.

LaKose, Laura. 2004. "Viewing landscapes through GIS: The Higuchi viewshed approach." MA, Geography, West Virginia University.

Lanigan, Richard L. 1988. Phenomenology of communication: Merleau-Ponty's thematics in communicology and semiology. Pittsburgh, PA: Duquesne University Press.

Llobera, Marcos. 1996. "Exploring the Topography of Mind: GIS, Social Space and Archaeology." Antiquity 70: 612-622.

Llobera, Marcos. 2001. "Building Past Landscape Perception With GIS: Understanding Geographic Prominence." Journal of Archaeological Science 28: 1005-1014.

Llobera, Marcos. 2003. "Extending GIS-based visual analysis: the concept of visualscapes." International Journal Geographical Information Science 17 (1): 25-48.

Lock, Gary, ed. 2000. Beyond the map: archaeology and spatial technologies. Washington, DC: IOS Press.

Lock, Gary. 2003. Using Computers in Archaeology: towards virtual pasts. New York: Routledge.

Lock, Gary, and Trevor M. Harris. 1996. "Danebury revisited: an Iron Age hillfort in a digital landscape." In Anthropology, Space, and Geographic Information and Analysis edited by H. D. G. Maschner, 214-240. London: Oxford University Press.

Lock, Gary, and Brian Leigh Molyneaux, eds. 2008. Confronting Scale in Archaeology: Issues of Theory and Practice. New York: Springer. 
Lock, Gary, and Zoran Stancic, eds. 1995. Archaeology and Geographical Information Systems. Bristol, PA: Taylor and Francis.

Longley, Paul, Michael F Goodchild, David J Maguire, and David W Rhind. 2005. Geographic Information Systems and Science. 2nd ed. Hoboken, NJ: John Wiley \& Sons.

Lowenthal, David. 1975. "Past Time, Present Place: Landscape and Memory." The Geographical Review 85 (1): 1-36.

Macann, C. E. 1993. Four Phenomenological Philosophers: Husserl, Heidegger, Sartre, Merleau-Ponty. New York: Routledge.

Madry, Scott L. H., and Lynn Rakos. 1996. "Line-of-Sight and Cost-Surface Techniques for Regional Research in Arroux River Valley." In New Methods, Old Problems: Geographic Information Systems in Modern Archaeological Research, edited by H. D. G. Maschner, 104126. Carbondale: Southern Illinois University at Carbondale.

Maguire, D. J., Michael Batty, and Michael F. Goodchild. 2005. GIS, spatial analysis, and modeling. Redlands: ESRI Press.

Malpas, Jeff. 1999. Place and Experience: A Philosophical Topography. New York: Cambridge University Press.

Malpas, Jeff. 2006. Heidegger's topology: being, place, world. Cambridge, MA: MIT Press. McEwan, Dorothy Graves, and Kirsty Millican. 2012. "In Search of the Middle Ground: Quantitative Spatial Techniques and Experiential Theory in Archaeology." Journal of Archaeological Method and Theory 19 (4): 491-494.

McHarg, Ian L., and Lewis Mumford. 1969. Design with nature. Garden City, N.Y.: American Museum of Natural History.

Merleau-Ponty, Maurice. 2002. Phenomenology of Perception. New York: Routledge.

Moran, Dermot. 2000. Introduction to Phenomenology. New York: Routledge.

Muir, Richard. 1981. The Shell Guide to Reading Landscape. London: Michael Joseph Ltd.

Murphy, James L. 1989. An archaeological history of the Hocking Valley. Athens: Ohio University Press.

Nash, George, ed. 1997. Semiotics of Landscape: Archaeology of Mind. Edited by Rajka Makjanic, BAR International Series. Oxford: Archaeopress.

Peet, Richard. 1998. Modern Geographical Thought. Malden, MA: Blackwell Publishing. 
Peregrine, Peter Neal, and Melvin Ember. 2002. "Adena." In Encyclopedia of Prehistory, Volume 3, 1-7. New York: Springer.

Pickles, John. 1985. Phenomenology, science, and geography: spatiality and the human sciences. New York: Cambridge University Press.

Pickles, John, ed. 1995. Ground Truth: The Social Implications of Geographic Information Systems. New York: Guilford Press.

Preston, Bryan, and Matthew W. Wilson. 2014. "Practicing GIS as Mixed Method: Affordances and Limitations in an Urban Gardening Study." Annals of the Association of American Geographers 104 (3): 510-529

Preucel, Robert W., ed. 1991. Processial and Postprocessual Archaeologies: Multiple Ways of Knowing the Past. Carbondale: Southern Illinois University.

Reilly, Paul, and Sebastian Rahtz, eds. 1992. Archaeology and the Information Age: a global perspective, One World Archaeology. New York: Routledge.

Relph, E. 1970. "An Inquiry into the Relations between Phenomenology and Geography." Canadian Geographer 14: 193-201.

Rennell, Rebecca. 2012. "Landscape, Experience, and GIS: Exploring the Potential for Methodological Dialogue." Journal of Archaeological Method and Theory 19 (4): 510- 525.

Riggs, Philip D, and Denis J Dean. 2007. "An Investigation into the Causes of Errors and Inconsistencies in Predicted Viewsheds." Transactions in GIS 11 (2): 175-196.

Rio Blanco County, CO. 2002. "Section 272: Standards for Viewscapes." Rio Blanco County Land Use Resolution

Rock, Irvin. 1995. Perception. New York: W.H. Freeman \& Company.

Rockmore, Tom. 2003. Before and after Hegel: a historical introduction to Hegel's thought. Indianapolis, IN: Hackett Publishing Company.

Romain, William G. 2005. "Appendix 3.1: Summary Report on the Orientations and Alignments of the Ohio Hopewell Geometric Enclosures." In Gathering Hopewell: Society, Ritual, and Ritual Interaction, edited by Christopher Carr and D. Troy Case. New York: Springer.

Rouse, L. Jesse. 1999. "Paradigm and Discourse: A look at the structure of knowledge in the social sciences." Biennial Conference on Appalachian Geography, Athens, WV. 
Rouse, L. Jesse. 2000. "Data Points or Cultural Entities: A GIS-based Archaeological Predictive Model in a Post-positivist Framework." MA, Geography, West Virginia University.

Rouse, L. Jesse, Susan J. Bergeron, and Trevor M. Harris. 2004. "Principles and applications of Lidar and laser scanning technologies in archaeology." Society for American Archaeology, Montreal.

Rouse, L. Jesse, Susan J. Bergeron, and Trevor M. Harris. 2007. "Participating in the Geospatial Web: Collaborative Mapping, Social Networks and Participatory GIS." In The Geospatial Web: How Geobrowsers, Social Software and the Web 2.0 are Shaping the Network Society, edited by Arno Scharl and Klaus Tochtermann, 153-158. New York: Springer.

Rouse, L. Jesse, Trevor M. Harris, and Susan J. Bergeron. 2004. "What is old is new again: the use of geospatial technologies for prehistoric landscapes reconstruction." International Geographical Congress, Glasgow.

Sargent, Winthrop. 1853. "Plan of an Ancient Fortification at Marietta, Ohio." Memoirs of the American Academy of Arts and Sciences 5 (1): 25-28.

Sartre, Jean-Paul. 1969. Being and nothingness: a phenomenological essay on ontology. Northampton: John Dickens \& Co.

Sassaman, Kenneth E. 2010. The Eastern Archaic, historicized, Issues in Eastern Woodlands archaeology. Lanham, MD: AltaMira Press.

Saturno, William, Thomans Sever, Daniel Irwin, Burgess Howell, and Thomas Garrison. 2007. "Putting Us on the Map: Remote Sensing Investigation of the Ancient Maya Landscape." In Remote Sensing in Archaeology, edited by James Wiseman and Farouk El-Baz, 137-160. New York: Springer.

Sauer, Carl O. 1944. "A Geographic Sketch of Early Man in America." Geographical Review 34 (4): 529-573.

Sauer, Carl Ortwin. 1925. The Morphology of Landscape. Berkeley: University of California Press.

Sauer, Carl Ortwin. 1963. "The Barrens of Kentucky." In Land and life: a selection from the writings of Carl Ortwin Sauer, edited by John Leighly, 23-31. Berkeley: University of California Press. 
Schuurman, Nadine. 2009. "Metadata as a site for imbuing GIS with qualitative information." In Qualitative GIS: A Mixed Methods Approach, edited by Meghan Cope and Sarah Elwood, 41-56. Thousand Oaks, CA: Sage Publications.

Schuurman, Nadine, and Agnieszka Leszczynski. 2006. "Ontology-Based Metadata."

Transactions in GIS 10 (5): 709-726.

Seamon, David, and Robert Mugerauer, eds. 1985. Dwelling, Place, and Environment: towards a phenomenology of person and world. New York: Columbia University Press.

Sheets, Payson D., and Brian R. McKee. 1994. Archaeology, Volcanism, and Remote Sensing in the Arenal Region, Costa Rica. Austin: University of Texas Press.

Silverberg, Robert. 1986. The Mound Builders. Athens: Ohio University Press.

Solomon, Jon, ed. 1993. Accessing Antiquity: The computerization of classical studies. Tucson: University of Arizona Press.

Spector, Janet. 1993. What this awl means: feminist archaeology at a Wahpeton Dakota village. St. Paul: Minnesota Historical Society Press.

Squire, Ephraim G., and Edwin B. Davis. 1998. Ancient Monuments of the Mississippi Valley. Edited by David J. Meltzer. 150th Anniversary ed. Washington: Smithsonian Books.

Soil Survey. 2011. U.S. General Soil Map. Natural Resources Conservation Service, United States Department of Agriculture.

Struever, Stuart. 1977. "The Hopewell Interaction Sphere in Riverine-Western Great Lakes Culture History." In Hopewellian Studies, edited by Joseph R. Caldwell and L. Robert Hall, 86-106. Springfield, IL: Illionois State Museum.

Sturtevant, Lynne. 2011. A Guide to Historic Marietta, Ohio. Charleston, SC: The History Press. Sui, Daniel, and Dydia DeLyser. 2011. "Crossing the qualitative-quantitative chasm I: Hybrid geographies, the spatial turn, and volunteered geographic information (VGI)." Progress in Human Geography 2011: 1-14.

Summers, Thomas J. 1903. History of Marietta. Marietta, OH: The Leader Publishing Co.

Tacon, Paul S. C. 1999. " Identifying Ancient Sacred Landscapes in Australia: From Physical to Social." In Archaeologies of Landscape: Contemporary Perspectives, edited by Wendy Ashmore and Arthur Bernard Knapp, 33-57. Malden MA: Blackwell. 
Taylor, Christopher, Paul Everson, and Tom Williamson, eds. 1998. The archaeology of landscape: Studies presented to Christopher Taylor. New York: Manchester University Press.

Taylor, Peter. 1990. "GKS." Political Geography Quarterly 9 (3): 211-212.

Taylor, Peter T., and M Overton. 1991. "Further thoughts on Geography and GIS." Environment and Planning A 23: 1087-1094.

Thomas, Julian. 1993. "The Hermeneutics of Megalithic Space." In Interpretative Archaeology, edited by Christopher Tilley, 73-98. Providence, RI: Berg.

Thomas, Julian. 1996. Time, Culture and Identity: an interpretive archaeology. New York: Routledge.

Thomas, Julian. 2004. Archaeology and Modernity. New York: Routledge.

Thomas, Julian, and Christopher Tilley. 1993. "The Axe and the Torso: Symbolic Structures in the Neolithic Brittany." In Interpretative Archaeology, edited by Christopher Tilley, 225-324. Providence, RI: Berg.

Thurston, Jeff, J. Patrick Moore, and Thomas K. Poiker. 2003. Integrated geospatial technologies: a guide to GPS, GIS, and data logging. Hoboken, N.J.: John Wiley \& Sons.

Tilley, Christopher. 1994. A Phenomenology of Landscape: places, paths and monuments. Providence, RI: Berg.

Tilley, Christopher. 2004. The materiality of stone: explorations in landscape phenomenology. New York: Berg.

Tilley, Christopher. 2010. Interpreting Landscapes: Geologies, Topographies, Identities. Walnut Creek, CA: Left Coast Press.

Tilley, Christopher, and Wayne Bennet. 2008. Body and Image: Explorations of Landscape Phenomenology 2. Walnut Creek, CA: Left Coast Press.

Trigger, Bruce. 1989. A history of archaeological thought. New York: Cambridge University Press.

Tschan, A. P., W. Raczkowski, and M. Latalowa. 2000. "Beyond the map: archaeology and spatial technologies." In Beyond the Map: Archaeology and Spatial Technologies, edited by Gary R. Lock, 28-48. Washington, DC: IOS Press. 
Tuan, Yi-Fu. 1974. Topophilia: A Study of Environmental Perception, Attitudes, and Values. Englewood Cliffs, NJ: Prentice Hall.

Tuan, Yi-fu. 1977. Space and place: the perspective of experience. Minneapolis: University of Minnesota Press.

Turner, Alasdair, Maria Doxa, David O'Sullivan, and Alan Penn. 2001. "From isovists to visibility graphs: a methodology for the analysis of architectural space." Environment and Planning B 28: 103-121.

USACE. 2013. "Muskingum Watershed Plan." http://www.lrh.usace.army.mil/Missions/CurrentProjects/MuskingumWatershedPlan.aspx (last accessed 10 September 2018)

van Dommelen, Peter. 1999. "Exploring Everyday Places and Cosmologies." In Archaeologies of Landscapes: Contemporary Perspectives, edited by Wendy Ashmore and A. Bernard Knapp, 277-285. Malden, MA: Blackwell.

van Hoven, Bettina. 2010. "Computer Assisted Qualitative Data Analysis." In Key Methods in Geography, edited by Nicholas Clifford, Shaun French and Gill Valentine, 453-465. Washington, DC: Sage.

Velarde-Mayol, Victor. 2000. On Husserl. Belmont, CA: Wadsworth.

Wade, Nicholas J., and Michael Swanston. 1991. Visual Perception: an introduction. New York: Routledge.

Weeson, Cameron B. 1998. "Mississippian Sacred Landscapes: A View from Alabama." In Mississippian Towns and Sacred Spaces: Searching for an Architectural Grammar, edited by R. Barry Lewis and Charles Stout. Tuscaloosa: University of Alabama Press.

Welton, Donn. 1999. The Essential Husserl: Basic Writings in Transcendental Phenomenology. Bloomington: Indiana University Press.

Wescott, Konnie, and R. Joe Brandon, eds. 2000. Practical applications of GIS for archaeologists: a predictive modeling toolkit. Philadelphia, PA: CRC Press.

Wheatley, D. 1996. "Anthropology, Space, and Geographic Information Systems." In New Methods, Old Problems: Geographic Information Systems in Modern Archaeological Research, edited by Mark Aldenderfer and Herbert D. G. Maschner, 75-103. Carbondale: Southern Illinois University at Carbondale. 
Wheatley, David. 1995. "Cumulative viewshed analysis: a GIS-based method for investigating intervisibility and its archaeological application." In Archaeology and Geographic Information Systems: A European Perspective, edited by Gary R Lock and Zoran Stancic, 171-185. Bristol, PA: CRC Press.

Wheatley, David, and Mark Gillings. 2000. "Beyond the map: archaeology and spatial technologies." In Beyond the Map: Archaeology and Spatial Technologies, edited by Gary Lock, 1-27. Washington, DC: IOS Press.

Wheatley, David, and Mark Gillings. 2002. Spatial Technology and Archaeology: The Archaeological Applications of GIS. New York: CRC Press.

Whitley, Thomas G. 2000. "Dynamical Systems Modeling in Archaeology: A GIS Approach to Site Selection Processes in the Greater Yellowstone Region." PhD, Anthropology, University of Pittsburgh.

Wiener, Jan M., and Gerald Franz. 2005. "Isovists as a Means to Predict Spatial Experience and Behavior." In Spatial Cognition IV. Reasoning, Action, Interaction, 42-57. New York: Springer.

Williamson, Christina G. 2016. "Mountain, Myth, and Territory: Teuthrania as Focal Point in the Landscape of Pergamon." In Valuing Landscape in Classical Antiquity: Natural Environment and Cultural Imagination, edited by Jeremy McInerney and Ineke Sluiter, 70-102. Boston, MA: Brill.

Wilson, Jeffrey, Gred Lindsey, and Gilbert Liu. 2008. "Viewshed characteristics of urban pedestrian trails, Indianapolis, Indiana, USA." Journal of Maps 2008: 108-118.

Wilson, Matthew W. 2009. "Towards a genealogy of qualitative GIS." In Qualitative GIS: $a$ mixed methods approach, edited by Meghan Cope and Sarah Elwood. Los Angeles, CA: Sage.

Wright, Dawn J., Michael F. Goodchild, and James D. Proctor. 1997. "Demystifying the Persistent Ambiguity of GIS as "Tool" Versus "Science"." Annals of the Association of American Geographers 87 (2): 346-362. 